\title{
THE SECOND COEFFICIENT OF THE ASYMPTOTIC EXPANSION OF THE WEIGHTED BERGMAN KERNEL FOR $(0, q)$ FORMS ON $\mathbb{C}^{n}$
}

\author{
CHIN-YU HSIAO
}

\author{
Communicated by Jih-Hsin Cheng
}

\begin{abstract}
Institute of Mathematics, Academia Sinica and National Center for Theoretical Sciences, 6F, AstronomyMathematics Building, No.1, Sec.4, Roosevelt Road, Taipei 10617, Taiwan.

E-mail: chsiao@math.sinica.edu.tw; chinyu.hsiao@gmail.com

\|\|$\|$
\end{abstract}

\begin{abstract}
Let $\phi \in C^{\infty}\left(\mathbb{C}^{n}\right)$ be a given real valued function. We assume that $\partial \bar{\partial} \phi$ is nondegenerate of constant signature $\left(n_{-}, n_{+}\right)$on $\mathbb{C}^{n}$. When $q=n_{-}$, it is well-known that the Bergman kernel for $(0, q)$ forms with respect to the $k$-th weight $e^{-2 k \phi}, k>0$, admits a full asymptotic expansion in $k$. In this paper, we compute the trace of the second coefficient of the asymptotic expansion on the diagonal.
\end{abstract}

\section{Introduction and Statement of the Main Result}

Let $L$ be a holomorphic line bundle over a Hermitian manifold $(M, \Theta)$, where $\Theta$ is a smooth positive $(1,1)$-form on $M$, and let $L^{k}$ be the $k$-th tensor power of $L$. Let $\square_{k}^{(q)}$ be the Gaffney extension of the Kodaira Laplacian acting on $(0, q)$ forms with values in $L^{k}$. The Bergman kernel is the distribution kernel of the orthogonal projection onto Ker $\square_{k}^{(q)}$ in the $L^{2}$ space. We assume that the curvature of $L$ is non-degenerate of constant signature $\left(n_{-}, n_{+}\right)$on $M$ and let $q=n_{-}$. When $M$ is compact, Catlin [2] and Zelditch [18] established the asymptotic expansion of the diagonal of the Bergman kernel

Received July 15, 2016 and in revised form August 7, 2016.

AMS Subject Classification: 58J40, 32C15.

Key words and phrases: Bergman kernel asymptotics, Kodaira Laplacian, the method of stationary phase.

The author was supported by Taiwan Ministry of Science of Technology project 104-2628-M-001003-MY2 and the Golden-Jade fellowship of Kenda Foundation. 
for $q=n_{-}=0$ and Berman-Sjöstrand [1], Ma-Marinescu [13] established the asymptotic expansion of the Bergman kernel for $q=n_{-} \geq 0$. When $M$ is complete and $L$ is uniformly positive on $M$ with $\sqrt{-1} R^{K_{M}^{*}}$ and $\partial \Theta$ bounded below, where $R^{K_{M}^{*}}$ is the curvature of the bundle of $(n, 0)$ forms, Ma-Marinescu [15] obtained the asymptotic expansion of the Bergman kernel for $q=n_{-}=0$. More generally, if $M$ is any complex manifold and $\square_{k}^{(q)}$ has $O\left(k^{-n_{0}}\right)$ small spectral gap on an open set $D \Subset M$ (see Definition 1.5 in [9], for the precise meaning of $O\left(k^{-n_{0}}\right)$ small spectral gap), then it is known by a recent result (see Theorem 1.6 in [9]) that the Bergman kernel admits a full asymptotic expansion in $k$ on $D$. The coefficients of these expansions turned out to be deeply related to various problem in complex geometry (see e.g. [3], [4], [5]).

The first four coefficients of the expansion of the Bergman kernel for $q=n_{-}=0$ on the diagonal were computed by $\mathrm{Lu}[12]$. The method of $\mathrm{Lu}$ is to construct appropriate peak sections as in [17], using Hörmander's $L^{2}$-method. Ma-Marinescu [16] calculated the first three coefficients of the expansion of the kernel of Berezin-Toeplitz quantization on the diagonal by using kernel calculations on $\mathbb{C}^{n}$. The author [8] gave a new method to calculated the first three coefficients of the expansion of the kernel of BerezinToeplitz quantization on the diagonal by using microlocal analysis. All these results are concern $q=n_{-}=0$.

In this paper, we give for the first time a formula of the second coefficient of the expansion of the Bergman kernel for $q=n_{-}>0$ on $\mathbb{C}^{n}$. We calculate the trace of the second coefficient of the expansion of the Bergman kernel for $q=n_{-}>0$ when $M=\mathbb{C}^{n}$ and $L$ is the trivial line bundle $\mathbb{C}$ endowed with the metric $|1|^{2}=e^{-2 \phi}$, where $\phi \in C^{\infty}\left(\mathbb{C}^{n}\right)$ is a given real valued function with $\partial \bar{\partial} \phi$ is non-degenerate of constant signature $\left(n_{-}, n_{+}\right)$on $\mathbb{C}^{n}$. There are two ingredients of our approach: the phase function version of the asymptotic expansion of the Bergman kernel and the method of stationary phase. Even through the calculation is quite complicate, the arguments in this paper are simple.

After the paper was completed Wen Lu [11] informed the author that he also obtained the formula for the coefficient $b_{1}$ by using the method of Ma-Marinescu [14]. Moreover, Wen Lu [11] obtained the formula for $b_{1}$ on general compact complex manifolds. 


\subsection{Notations}

Let $\Omega$ be a $C^{\infty}$ paracompact manifold equipped with a smooth density of integration. We let $T(\Omega)$ and $T^{*}(\Omega)$ denote the tangent bundle of $\Omega$ and the cotangent bundle of $\Omega$ respectively. The complexified tangent bundle of $\Omega$ and the complexified cotangent bundle of $\Omega$ will be denoted by $\mathbb{C} T(\Omega)$ and $\mathbb{C} T^{*}(\Omega)$ respectively. We write $<,>$ to denote the pointwise duality between $T(\Omega)$ and $T^{*}(\Omega)$. We extend $<,>$ bilinearly to $\mathbb{C} T(\Omega) \times \mathbb{C} T^{*}(\Omega)$. Let $E$ be a $C^{\infty}$ vector bundle over $\Omega$. The fiber of $E$ at $x \in \Omega$ will be denoted by $E_{x}$. Let $Y \subset \subset \Omega$ be an open set. From now on, the spaces of smooth sections of $E$ over $Y$ and distribution sections of $E$ over $Y$ will be denoted by $C^{\infty}(Y ; E)$ and $\mathscr{D}^{\prime}(Y ; E)$ respectively. Let $\mathscr{E}^{\prime}(Y ; E)$ be the subspace of $\mathscr{D}^{\prime}(Y ; E)$ whose elements have compact support in $Y$. Put $C_{0}^{\infty}(Y ; E)=C^{\infty}(Y ; E) \bigcap \mathscr{E}^{\prime}(Y ; E)$. We let $L^{2}(Y ; E)$ denote the $L^{2}$ space of sections of $E$ over $Y$.

We shall denote the real coordinates by $x_{j}, j=1, \ldots, 2 n$, and the complex coordinates by $z=\left(z_{1}, \ldots, z_{n}\right), z_{j}=x_{2 j-1}+i x_{2 j}, j=1, \ldots, n$. Let $\Lambda^{1,0} T\left(\mathbb{C}^{n}\right)$ and $\Lambda^{0,1} T\left(\mathbb{C}^{n}\right)$ denote the holomorphic tangent bundle and the anti-holomorphic tangent bundle of $\mathbb{C}^{n}$ respectively. We take the Hermitian metric $(\mid)$ on $\mathbb{C} T\left(\mathbb{C}^{n}\right)$ such that $\left(\frac{\partial}{\partial z_{j}} \mid \frac{\partial}{\partial z_{k}}\right)=\left(\frac{\partial}{\partial \bar{z}_{j}} \mid \frac{\partial}{\partial \bar{z}_{k}}\right)=$ $\delta_{j, k}, j, k=1, \ldots, n, \Lambda^{1,0} T\left(\mathbb{C}^{n}\right) \perp \Lambda^{0,1} T\left(\mathbb{C}^{n}\right)$, where $\frac{\partial}{\partial z_{j}}=\frac{1}{2}\left(\frac{\partial}{\partial x_{2 j-1}}-i \frac{\partial}{\partial x_{2 j}}\right)$, $\frac{\partial}{\partial \bar{z}_{j}}=\frac{1}{2}\left(\frac{\partial}{\partial x_{2 j-1}}+i \frac{\partial}{\partial x_{2 j}}\right), j=1, \ldots, n$, and $\delta_{j, k}=1$ if $j=k, \delta_{j, k}=0$ if $j \neq k$. For $p, q \geq 0, p, q \in \mathbb{Z}$, let $\Lambda^{p, q} T^{*}\left(\mathbb{C}^{n}\right)$ be the bundle of $(p, q)$ forms of $\mathbb{C}^{n}$. We say that a multiindex $J=\left(j_{1}, \ldots, j_{q}\right) \in\{1, \ldots, n-1\}^{q}$ has length $q$ and write $|J|=q$. We say that $J$ is strictly increasing if $1 \leqslant j_{1}<j_{2}<\cdots<j_{q} \leqslant n-1$. For multiindices $J=\left(j_{1}, \ldots, j_{q}\right)$, $K=\left(k_{1}, \ldots, k_{p}\right)$, we define $d z_{K} \wedge d \bar{z}_{J}:=d z_{k_{1}} \wedge \cdots \wedge d z_{k_{p}} \wedge d \bar{z}_{j_{1}} \wedge \cdots \wedge d \bar{z}_{j_{q}}$. We take the Hermitian metric $(\mid)$ on $\Lambda^{p, q} T^{*}\left(\mathbb{C}^{n}\right)$ so that $\left\{d z_{K} \wedge d \bar{z}_{J}\right.$ : $|K|=p,|J|=q, K, J$ are strictly increasing $\}$ is an orthonormal frame for $\Lambda^{p, q} T^{*}\left(\mathbb{C}^{n}\right)$. Let $T \in \mathscr{L}\left(\Lambda^{p, q} T^{*}\left(\mathbb{C}^{n}\right), \Lambda^{p, q} T^{*}\left(\mathbb{C}^{n}\right)\right)$. Then the trace of $T$ is given by

$$
\operatorname{Tr} T=\sum_{|K|=p,|J|=q}^{\prime}\left(T\left(d z_{K} \wedge d \bar{z}_{J}\right) \mid d z_{K} \wedge d \bar{z}_{J}\right)
$$


where $\sum^{\prime}$ means that the summation is performed only over strictly increasing multiindices. Thus $\operatorname{Tr} T=0$ if

$$
\left(T\left(d z_{K} \wedge d \bar{z}_{J}\right) \mid d z_{K} \wedge d \bar{z}_{J}\right)=0
$$

for all strictly increasing multiinindices $K, J,|K|=p,|J|=q$.

If $w \in \Lambda^{0,1} T_{z}^{*}\left(\mathbb{C}^{n}\right)$, let $w^{\wedge, *}: \Lambda^{0, q+1} T_{z}^{*}\left(\mathbb{C}^{n}\right) \rightarrow \Lambda^{0, q} T_{z}^{*}\left(\mathbb{C}^{n}\right)$ be the adjoint of left exterior multiplication $w^{\wedge}: \Lambda^{0, q} T_{z}^{*}\left(\mathbb{C}^{n}\right) \rightarrow \Lambda^{0, q+1} T_{z}^{*}\left(\mathbb{C}^{n}\right)$. That is,

$$
\left(w^{\wedge} u \mid v\right)=\left(u \mid w^{\wedge, *} v\right)
$$

for all $u \in \Lambda^{0, q} T_{z}^{*}\left(\mathbb{C}^{n}\right), v \in \Lambda^{0, q+1} T_{z}^{*}\left(\mathbb{C}^{n}\right)$. Notice that $w^{\wedge, *}$ depends antilinearly on $w$.

Let $E, F$ be $C^{\infty}$ vector bundles over a smooth manifold $M$. We say that a $k$-dependent function $f(x, y, k) \in C^{\infty}\left(M \times M ; \mathscr{L}\left(E_{y}, F_{x}\right)\right)$ is negligible if for every compact set $K \subset M \times M$ and for all $N>0$ and multiindice $\alpha, \beta$, there is a constant $c_{N, \alpha, \beta, K}>0$ independent of $k$ such that for $k$ sufficiently large, $\left|\partial_{x}^{\alpha} \partial_{y}^{\beta} f(x, y, k)\right| \leq c_{\alpha, \beta, K} k^{-N},(x, y) \in K$. Let $b(x, y, k) \in$ $C^{\infty}\left(M \times M ; \mathscr{L}\left(E_{y}, F_{x}\right)\right)$ be a $k$-dependent smooth function. We write

$$
b \sim \sum_{0}^{\infty} b_{j}(x, y) k^{-N_{0}-j}
$$

in $C^{\infty}\left(M \times M ; \mathscr{L}\left(E_{y}, F_{x}\right)\right), b_{j}(x, y) \in C^{\infty}\left(M \times M ; \mathscr{L}\left(E_{y}, F_{x}\right)\right), j=0,1, \ldots$, if for all $M_{0} \in \mathbb{N}$, every compact set $K \subset M \times M$ and for all multiindice $\alpha, \beta$, there is a constant $c_{M_{0}, \alpha, \beta, K}>0$ independent of $k$ such that for $k$ sufficiently large,

$$
\left|\partial_{x}^{\alpha} \partial_{y}^{\beta}\left(b-\sum_{0}^{M_{0}} b_{j}(x, y) k^{-N_{0}-j}\right)\right| \leq c_{M_{0}, \alpha, \beta, K} k^{-N_{0}-M_{0}-1},
$$

$(x, y) \in K$.

\subsection{The asymptotic expansion of the Bergman kernel}

Let $\phi(z) \in C^{\infty}\left(\mathbb{C}^{n} ; \mathbb{R}\right)$. In this work we assume that $\left(\frac{\partial^{2} \phi}{\partial \bar{z}_{j} \partial z_{k}}\right)_{j, k=1}^{n}$ is non-degenerate of constant signatute $\left(n_{-}, n_{+}\right)$. That is, the number of 
negative eigenvalues of $\left(\frac{\partial^{2} \phi}{\partial \bar{z}_{j} \partial z_{k}}\right)_{j, k=1}^{n}$ is $n_{-}$and $n_{-}+n_{+}=n$.

We take $d m=2^{n} d x_{1} d x_{2} \cdots d x_{2 n}$ as the volume form on $\mathbb{C}^{n}$. Let $(\mid)$ be the inner product on $C_{0}^{\infty}\left(\mathbb{C}^{n} ; \Lambda^{0, q} T^{*}\left(\mathbb{C}^{n}\right)\right)$ defined by

$$
(f \mid g)=\int_{\mathbb{C}^{n}}(f(z) \mid g(z))(d m), \quad f, g \in C_{0}^{\infty}\left(\mathbb{C}^{n} ; \Lambda^{0, q} T^{*}\left(\mathbb{C}^{n}\right)\right) .
$$

For $k>0$, let $(\mid)_{k}$ be the inner product on $C_{0}^{\infty}\left(\mathbb{C}^{n} ; \Lambda^{0, q} T^{*}\left(\mathbb{C}^{n}\right)\right)$ defined by

$$
(f \mid g)_{k}=\int_{\mathbb{C}^{n}}(f(z) \mid g(z)) e^{-2 k \phi(z)}(d m), f, g \in C_{0}^{\infty}\left(\mathbb{C}^{n} ; \Lambda^{0, q} T^{*}\left(\mathbb{C}^{n}\right)\right) .
$$

Let $L_{q}^{2}\left(\mathbb{C}^{n}\right)$ and $L_{q, k}^{2}\left(\mathbb{C}^{n}\right)$ be the completions of $C_{0}^{\infty}\left(\mathbb{C}^{n} ; \Lambda^{0, q} T^{*}\left(\mathbb{C}^{n}\right)\right)$ with respect to $(\mid)$ and $(\mid)_{k}$ respectively. We extend the $L^{2}$ inner products $(\mid)$ and $(\mid)_{k}$ to $L_{q}^{2}\left(\mathbb{C}^{n}\right)$ and $L_{q, k}^{2}\left(\mathbb{C}^{n}\right)$ respectively.

Let $\bar{\partial}: C^{\infty}\left(\mathbb{C}^{n} ; \Lambda^{0, q} T^{*}\left(\mathbb{C}^{n}\right)\right) \rightarrow C^{\infty}\left(\mathbb{C}^{n} ; \Lambda^{0, q+1} T^{*}\left(\mathbb{C}^{n}\right)\right)$ be the part of the exterior differential operator which maps forms of type $(0, q)$ to forms of type $(0, q+1)$. We extend $\bar{\partial}$ to $L_{q, k}^{2}\left(\mathbb{C}^{n}\right)$ by

$$
\bar{\partial}_{k}: \operatorname{Dom} \bar{\partial}_{k} \subset L_{q, k}^{2}\left(\mathbb{C}^{n}\right) \rightarrow L_{q+1, k}^{2}\left(\mathbb{C}^{n}\right)
$$

where Dom $\bar{\partial}_{k}:=\left\{u \in L_{q, k}^{2}\left(\mathbb{C}^{n}\right) ; \bar{\partial} u \in L_{q+1, k}^{2}\left(\mathbb{C}^{n}\right)\right\}$, where $\bar{\partial} u$ is defined in the sense of distributions. We write

$$
\bar{\partial}_{k}^{*}: \operatorname{Dom} \bar{\partial}_{k}^{*} \subset L_{q+1, k}^{2}\left(\mathbb{C}^{n}\right) \rightarrow L_{q, k}^{2}\left(\mathbb{C}^{n}\right)
$$

to denote the Hilbert space adjoint of $\bar{\partial}_{k}$ in the $L^{2}$ space with respect to $(\mid)_{k}$. Let $\square_{k}^{(q)}$ denote the Gaffney extension of the Kodaira Laplacian given by

$$
\square_{k}^{(q)}=\bar{\partial}_{k} \bar{\partial}_{k}^{*}+\bar{\partial}_{k}^{*} \bar{\partial}_{k}: \operatorname{Dom} \square_{k}^{(q)} \subset L_{q, k}^{2}\left(\mathbb{C}^{n}\right) \rightarrow L_{q, k}^{2}\left(\mathbb{C}^{n}\right),
$$

where

$$
\begin{array}{r}
\operatorname{Dom} \square_{k}^{(q)}=\left\{s \in L_{q, k}^{2}\left(\mathbb{C}^{n}\right) ; s \in \operatorname{Dom} \bar{\partial}_{k} \cap \operatorname{Dom} \bar{\partial}_{k}^{*}, \bar{\partial}_{k} u \in \operatorname{Dom} \bar{\partial}_{k}^{*},\right. \\
\left.\bar{\partial}_{k}^{*} u \in \operatorname{Dom} \bar{\partial}_{k}\right\} .
\end{array}
$$

By a result of Gaffney [14, Prop. 3.1.2], $\square_{k}^{(q)}$ is a positive self-adjoint operator. 
Let

$$
\Pi_{k}^{(q)}: L_{q, k}^{2}\left(\mathbb{C}^{n}\right) \rightarrow \operatorname{Ker} \square_{k}^{(q)}
$$

be the Bergman projection, i.e. the orthogonal projection onto $\operatorname{Ker} \square_{k}^{(q)}$ with respect to $(\mid)_{k}$ and let $\Pi_{k}^{(q)}(z, w)$ be the distribution kernel of $\Pi_{k}^{(q)}$ with respect to the volume form $d m$. Since $\square_{k}^{(q)}$ is elliptic, it is not difficult to see that

$$
\Pi_{k}^{(q)}(z, w) \in C^{\infty}\left(\mathbb{C}^{n} \times \mathbb{C}^{n} ; \mathscr{L}\left(\Lambda^{0, q} T^{*}\left(\mathbb{C}^{n}\right), \Lambda^{0, q} T^{*}\left(\mathbb{C}^{n}\right)\right)\right.
$$

We write

$$
\Pi_{k}^{(q)} u(z)=\int_{\mathbb{C}^{n}} \Pi_{k}^{(q)}(z, w) u(w) d m(w),
$$

$u \in C_{0}^{\infty}\left(\mathbb{C}^{n} ; \Lambda^{0, q} T^{*}\left(\mathbb{C}^{n}\right)\right)$.

It is well-known that $\square_{k}^{(q)}$ has $O\left(k^{-n_{0}}\right)$ small spectral gap on every open set $D \Subset M$ (see Definition 1.5 in [9], for the precise meaning of $O\left(k^{-n_{0}}\right)$ small spectral gap). From this observation and Theorem 4.12, Theorem 4.14 in [9], we deduce the following

Theorem 1.1. Let $D \Subset \mathbb{C}^{n}$ be an open set and let $0 \leq q \leq n$. If $q \neq n_{-}$, then $e^{-k \phi(z)} \Pi_{k}^{(q)}(z, w) e^{k \phi(w)}$ is negligible on $D \times D$. If $q=n_{-}$, then

$$
\Pi_{k}^{(q)} u(z)=\int_{\mathbb{C}^{n}} e^{i k \psi(z, w)} e^{k(\phi(z)-\phi(w))} b(z, w, k) u(w) d m(w)+R u
$$

for $z \in D, u \in C_{0}^{\infty}\left(D, \Lambda^{0, q} T^{*}\left(\mathbb{C}^{n}\right)\right)$, where

$$
b \sim \sum_{0}^{\infty} b_{j}(z, w) k^{n-j}
$$

in $C^{\infty}\left(D \times D ; \mathscr{L}\left(\Lambda^{0, q} T_{w}^{*}\left(\mathbb{C}^{n}\right), \Lambda^{0, q} T_{z}^{*}\left(\mathbb{C}^{n}\right)\right)\right)$,

$$
b_{j} \in C^{\infty}\left(D \times D ; \mathscr{L}\left(\Lambda^{0, q} T_{w}^{*}\left(\mathbb{C}^{n}\right), \Lambda^{0, q} T_{z}^{*}\left(\mathbb{C}^{n}\right)\right)\right),
$$

$j=0,1, \ldots$,

$$
R u=\int_{\mathbb{C}^{n}} e^{k(\phi(z)-\phi(w))} r(z, w, k) u(w) d m(w),
$$


$r(z, w, k)$ is negligible and

$$
\begin{aligned}
& \psi \in C^{\infty}\left(\mathbb{C}^{n} \times \mathbb{C}^{n}\right), \quad \psi(z, z)=0, \quad \psi(z, w)=-\bar{\psi}(w, z), \\
& \operatorname{Im} \psi(z, w) \geq c|z-w|^{2}, c>0 .
\end{aligned}
$$

For $z=w$, we have

$$
\frac{\partial \psi}{\partial \bar{z}}=i \frac{\partial \phi}{\partial \bar{z}}, \quad \frac{\partial \psi}{\partial z}=-i \frac{\partial \phi}{\partial z}, \quad \frac{\partial \psi}{\partial \bar{w}}=-i \frac{\partial \phi}{\partial \bar{z}}, \quad \frac{\partial \psi}{\partial w}=i \frac{\partial \phi}{\partial z}
$$

Moreover,

$$
\sum_{j=1}^{n}\left(i \frac{\partial \psi(z, w)}{\partial \bar{z}_{j}}+\frac{\partial \phi(z)}{\partial \bar{z}_{j}}\right)\left(-i \frac{\partial \psi(z, w)}{\partial z_{j}}+\frac{\partial \phi(z)}{\partial z_{j}}\right)
$$

vanishes to infinity order on $z=w$. Furthermore, the Taylor expansion of the phase $\psi(z, w)$ is uniquely determined at each point of $z=w$.

In particular,

$$
\begin{array}{r}
\Pi_{k}^{(q)}(z, z) \sim k^{n} b_{0}(z, z)+k^{n-1} b_{1}(z, z)+k^{n-2} b_{2}(z, z)+\cdots \\
\text { locally uniformly on } \mathbb{C}^{n} .
\end{array}
$$

The leading term $b_{0}(z, z)$ is essentially well-known (see formula (1.24) in Ma-Marinescu [13]).

Theorem 1.2. Let $q=n_{-}$. For $p \in \mathbb{C}^{n}$, we assume that $\lambda_{j}(p), j=1, \ldots, n$, are the eigenvalues of $\left(\frac{\partial^{2} \phi}{\partial \bar{z}_{j} \partial z_{k}}(p)\right)_{j, k=1}^{n}$ with respect to $(\mid)$ and that $\lambda_{j}(p)<0$ if $1 \leq j \leq n_{-}$. Let $U_{1}(p), \ldots, U_{n}(p)$ be an orthonormal basis of $\Lambda^{1,0} T_{p}\left(\mathbb{C}^{n}\right)$ such that $<\partial \bar{\partial} \phi(p), U_{s}(p) \wedge \bar{U}_{t}(p)>=\delta_{s, t} \lambda_{s}(p), s, t=1, \ldots, n$. Let $\bar{U}_{j}^{*}(p)$, $j=1, \ldots, n$, denote the orthonormal basis of $\Lambda^{0,1} T_{p}^{*}\left(\mathbb{C}^{n}\right)$, which is dual to $\bar{U}_{j}(p), j=1, \ldots, n$. Then,

$$
b_{0}(p, p)=\left|\lambda_{1}(p)\right|\left|\lambda_{2}(p)\right| \cdots\left|\lambda_{n}(p)\right| \pi^{-n} \prod_{j=1}^{j=n_{-}} \bar{U}_{j}^{*}(p)^{\wedge} \bar{U}_{j}^{*}(p)^{\wedge, *} .
$$




\subsection{The main result}

In order to state our result precisely, we have to introduce some notations and definitions. Let

$$
F: \Lambda^{1,0} T\left(\mathbb{C}^{n}\right) \rightarrow \Lambda^{1,0} T\left(\mathbb{C}^{n}\right) \otimes \Lambda^{p, q} T^{*}\left(\mathbb{C}^{n}\right)
$$

be a linear operaor, where $p, q \in \mathbb{Z}, p, q \geq 0$. We write $F=\left(F_{j, k}\right)_{j, k=1}^{n}$, $F_{j, k} \in \Lambda^{p, q} T^{*}\left(\mathbb{C}^{n}\right), 1 \leq j, k \leq n$,

$$
F \frac{\partial}{\partial z_{k}}=\sum_{j=1}^{n} \frac{\partial}{\partial z_{j}} \otimes F_{j, k}
$$

$k=1, \ldots, n$. We have

$$
(F U \mid V)=\sum_{j, k=1}^{n} u_{k} \bar{v}_{j} F_{j, k} \in \Lambda^{p, q} T^{*}\left(\mathbb{C}^{n}\right),
$$

where $U=\sum_{k=1}^{n} u_{k} \frac{\partial}{\partial z_{k}}, V=\sum_{j=1}^{n} v_{j} \frac{\partial}{\partial z_{j}} \in \Lambda^{1,0} T\left(\mathbb{C}^{n}\right)$.

Let $\left.T: \Lambda^{1,0} T\left(\mathbb{C}^{n}\right)\right) \rightarrow \Lambda^{1,0} T\left(\mathbb{C}^{n}\right) \otimes \Lambda^{r, t} T^{*}\left(\mathbb{C}^{n}\right)$ be another linear operator, where $r, t \in \mathbb{Z}, r, s \geq 0$. We write $T=\left(T_{j, k}\right)_{j, k=1}^{n}, T_{j, k} \in \Lambda^{r, t} T^{*}\left(\mathbb{C}^{n}\right)$, $j, k=1, \ldots, n$, as in (1.17). Then

$$
T F: \Lambda^{1,0} T\left(\mathbb{C}^{n}\right) \rightarrow \Lambda^{1,0} T\left(\mathbb{C}^{n}\right) \otimes \Lambda^{p+r, q+t} T^{*}\left(\mathbb{C}^{n}\right)
$$

is the linear operator defined by $T F \frac{\partial}{\partial z_{k}}=\sum_{s, j=1}^{n} \frac{\partial}{\partial z_{s}} \otimes\left(T_{s, j} \wedge F_{j, k}\right), k=$ $1, \ldots, n$.

We assume that $F$ is smooth. That is,

$$
F: C^{\infty}\left(\mathbb{C}^{n} ; \Lambda^{1,0} T\left(\mathbb{C}^{n}\right)\right) \rightarrow C^{\infty}\left(\mathbb{C}^{n} ; \Lambda^{1,0} T\left(\mathbb{C}^{n}\right) \otimes \Lambda^{p, q} T^{*}\left(\mathbb{C}^{n}\right)\right)
$$

Let

$$
\bar{\partial} F: C^{\infty}\left(\mathbb{C}^{n} ; \Lambda^{1,0} T\left(\mathbb{C}^{n}\right)\right) \rightarrow C^{\infty}\left(\mathbb{C}^{n} ; \Lambda^{1,0} T\left(\mathbb{C}^{n}\right) \otimes \Lambda^{p, q+1} T^{*}\left(\mathbb{C}^{n}\right)\right)
$$

be the smooth linear operator defined by $\bar{\partial} F \frac{\partial}{\partial z_{k}}=\sum_{j=1}^{n} \frac{\partial}{\partial z_{j}} \otimes\left(\bar{\partial} F_{j, k}\right), k=$ $1, \ldots, n$. Similarly,

$$
\partial F: C^{\infty}\left(\mathbb{C}^{n} ; \Lambda^{1,0} T\left(\mathbb{C}^{n}\right)\right) \rightarrow C^{\infty}\left(\mathbb{C}^{n} ; \Lambda^{1,0} T\left(\mathbb{C}^{n}\right) \otimes \Lambda^{p+1, q} T^{*}\left(\mathbb{C}^{n}\right)\right)
$$


is the smooth linear operator defined by $\partial F \frac{\partial}{\partial z_{k}}=\sum_{j=1}^{n} \frac{\partial}{\partial z_{j}} \otimes\left(\partial F_{j, k}\right), k=$ $1, \ldots, n$.

Let

$$
M_{\phi}: C^{\infty}\left(\mathbb{C}^{n} ; \Lambda^{1,0} T\left(\mathbb{C}^{n}\right)\right) \rightarrow C^{\infty}\left(\mathbb{C}^{n} ; \Lambda^{1,0} T\left(\mathbb{C}^{n}\right)\right)
$$

be the smooth linear map defined by

$$
\left(M_{\phi} U \mid V\right)=<\partial \bar{\partial} \phi, U \wedge \bar{V}>
$$

$U, V \in C^{\infty}\left(\mathbb{C}^{n} ; \Lambda^{1,0} T\left(\mathbb{C}^{n}\right)\right)$. Note that $M_{\phi}=\left(\frac{\partial^{2} \phi}{\partial \bar{z}_{j} \partial z_{k}}\right)_{j, k=1}^{n}$ in the sense of (1.16). We write $M_{\phi}^{-1}: C^{\infty}\left(\mathbb{C}^{n} ; \Lambda^{1,0} T\left(\mathbb{C}^{n}\right)\right) \rightarrow C^{\infty}\left(\mathbb{C}^{n} ; \Lambda^{1,0} T\left(\mathbb{C}^{n}\right)\right)$ to denote the inverse of $M_{\phi}$.

We recall that we work with the assumption that $M_{\phi}$ is non-degenerate of constant signature $\left(n_{-}, n_{+}\right)$. For $z \in \mathbb{C}^{n}$, we can diagonalize $M_{\phi}(z)$, i.e. we can find an orthonormal basis $\left\{U_{j}\right\}_{j=1}^{n}$ of $\Lambda^{1,0} T\left(\mathbb{C}^{n}\right)$ such that $M_{\phi}(z) U_{j}(z)=\lambda_{j}(z) U_{j}(z), j=1, \ldots, n, \lambda_{j}(z) \in \mathbb{R}, j=1, \ldots, n$. From now on, we assume that

$$
\begin{aligned}
& M_{\phi}(z) U_{j}(z)=\lambda_{j}(z) U_{j}(z), \quad U_{j}(z) \in \Lambda^{1,0} T_{z}\left(\mathbb{C}^{n}\right), \quad\left(U_{j} \mid U_{j}\right)=1, \quad j=1, \ldots, n, \\
& \lambda_{j}(z)<0, j=1, \ldots, n_{-}, \quad \lambda_{j}(z)>0, j=n_{-}+1, \ldots, n .
\end{aligned}
$$

Let $W_{+}$be the subbundle of $\Lambda^{1,0} T\left(\mathbb{C}^{n}\right)$ spanned by $\left\{U_{n_{-}+1}, \ldots, U_{n}\right\}$ and let $W_{-}$be the subbundle of $\Lambda^{1,0} T\left(\mathbb{C}^{n}\right)$ spanned by $\left\{U_{1}, \ldots, U_{n_{-}}\right\}$. We take the Hermitian metric $(\mid)_{|\phi|}$ on $\Lambda^{1,0} T\left(\mathbb{C}^{n}\right)$ such that $W_{+} \perp W_{-},(U \mid V)_{|\phi|}=$ $\left(M_{\phi} U \mid V\right)$ if $U, V \in W_{+},(U \mid V)_{|\phi|}=-\left(M_{\phi} U \mid V\right)$ if $U, V \in W_{-}$. The Hermitian metric $(\mid)_{|\phi|}$ on $\mathbb{C} T\left(\mathbb{C}^{n}\right)$ induces a Hermitian metric on $\Lambda^{p, q} T^{*}\left(\mathbb{C}^{n}\right)$ also denoted by $(\mid)_{|\phi|}$. We take the Hermitian metric $(\mid)_{|\phi|}$ on $\Lambda^{1,0} T\left(\mathbb{C}^{n}\right)$ $\mathbb{C} T\left(\mathbb{C}^{n}\right)$.

The two form $\partial \bar{\partial} \phi$ induces a connection $D_{\phi}$ on the bundle $\Lambda^{1,0} T\left(\mathbb{C}^{n}\right)$ :

$$
\begin{aligned}
& D_{\phi}=d+\theta: C^{\infty}\left(\mathbb{C}^{n} ; \Lambda^{1,0}\left(\mathbb{C}^{n}\right)\right) \rightarrow C^{\infty}\left(\mathbb{C}^{n} ; \mathbb{C} T^{*}\left(\mathbb{C}^{n}\right) \otimes \Lambda^{1,0} T\left(\mathbb{C}^{n}\right)\right), \\
& D_{\phi}(\xi)=\sum_{j=1}^{n}\left(d \xi_{j}\right) \otimes \frac{\partial}{\partial z_{j}}+\sum_{1 \leq j, k \leq n} \theta_{j, k} \xi_{k} \otimes \frac{\partial}{\partial z_{j}}
\end{aligned}
$$

where $\xi=\sum_{j=1}^{n} \xi_{j} \frac{\partial}{\partial z_{j}}$ and $\theta=h^{-1} \partial h=\left(\theta_{j, k}\right)_{j, k=1}^{n}, h=\left(\frac{\partial^{2} \phi}{\partial \bar{z}_{j} \partial z_{k}}\right)_{j, k=1}^{n}$. We call $\theta$ the connection matrix for $\partial \bar{\partial} \phi$. The curvature of the connection $D_{\phi}$ is 
given by

$$
\begin{aligned}
& \Theta_{\phi}=\bar{\partial} \theta=\left(\bar{\partial} \theta_{j, k}\right)_{j, k=1}^{n}=\left(\Theta_{j, k}\right)_{j, k=1}^{n}, \\
& \Theta_{\phi}: C^{\infty}\left(\mathbb{C}^{n} ; \Lambda^{1,0} T\left(\mathbb{C}^{n}\right)\right) \rightarrow C^{\infty}\left(\mathbb{C}^{n} ; \Lambda^{1,1} T^{*}\left(\mathbb{C}^{n}\right) \otimes \Lambda^{1,0} T\left(\mathbb{C}^{n}\right)\right), \\
& \xi=\sum_{j=1}^{n} \xi_{j} \frac{\partial}{\partial z_{j}} \rightarrow \sum_{j, k=1}^{n} \Theta_{j, k} \xi_{k} \otimes \frac{\partial}{\partial z_{j}}
\end{aligned}
$$

For $j=1, \ldots, n$, define

$$
\begin{aligned}
& \delta_{j}(k)=0 \text { if }\{j, k\} \subset\{1, \ldots, q\} \text { or }\{j, k\} \subset\{q+1, \ldots, n\} \\
& \delta_{j}(k)=1 \text { otherwise. }
\end{aligned}
$$

Define

$$
\begin{aligned}
& Q: \Lambda^{1,0} T\left(\mathbb{C}^{n}\right) \rightarrow \Lambda^{1,0} T^{*}\left(\mathbb{C}^{n}\right) \otimes \Lambda^{1,0} T\left(\mathbb{C}^{n}\right), \\
& <\left(Q U_{j} \mid U_{k}\right), U_{s}>=\left(\frac{\left|\lambda_{j}\right| \delta_{k}(j)+\left|\lambda_{s}\right| \delta_{k}(s)}{\left|\lambda_{k}\right|+\left|\lambda_{j}\right| \delta_{k}(j)+\left|\lambda_{s}\right| \delta_{k}(s)}-\delta_{k}(j) \delta_{k}(s) \times\right. \\
& \left.\frac{\left|\lambda_{j}\right|^{2}\left|\lambda_{s}\right|^{2}}{\left(\left|\lambda_{j}\right|+\left|\lambda_{k}\right|+\left|\lambda_{s}\right|\right)^{2}}\left(\frac{1}{\left|\lambda_{j}\right|+\left|\lambda_{k}\right|}+\frac{1}{\left|\lambda_{k}\right|+\left|\lambda_{s}\right|}\right)^{2}\right)<\left(\partial M_{\phi} U_{j} \mid U_{k}\right), U_{s}>.
\end{aligned}
$$

It is not difficult to see that the definition (1.26) is independent of the choices of eigenvectors $U_{1}, \ldots, U_{n}$.

Define

$$
R=\Theta_{\phi}-\left(\bar{\partial} M_{\phi}^{-1}\right) Q: \Lambda^{1,0} T\left(\mathbb{C}^{n}\right) \rightarrow \Lambda^{1,1} T^{*}\left(\mathbb{C}^{n}\right) \otimes \Lambda^{1,0} T\left(\mathbb{C}^{n}\right) .
$$

Put $e_{j}=\frac{1}{\sqrt{\left|\lambda_{j}\right|}} U_{j}, j=1, \ldots, n$, where $U_{j}, j=1, \ldots, n$, are as in (1.22). The main result of this work is the following

Theorem 1.3. Under the assumptions and notations above, let $q=n_{-}$. For $p \in \mathbb{C}^{n}$ and for $b_{1}$ in (1.14), we have

$$
\begin{aligned}
\operatorname{Tr} b_{1}(p, p)= & 2^{n}\left|\lambda_{1}(p)\right|\left|\lambda_{2}(p)\right| \cdots\left|\lambda_{n}(p)\right| \pi^{-n} \\
& \times\left(\sum_{1 \leq k \leq q, q+1 \leq j \leq n, 1 \leq s \leq n} a_{j, k, s}(p)<\left(\partial M_{\phi} U_{j} \mid U_{k}\right), U_{s}>^{2}(p)\right.
\end{aligned}
$$




$$
\begin{aligned}
& +\frac{1}{4} \sum_{j, k=1}^{n}\left(1+\delta_{j}(k) \frac{\left|\lambda_{j}\right|-\left|\lambda_{k}\right|}{\left|\lambda_{j}\right|+\left|\lambda_{k}\right|}\right)<\left(R e_{j} \mid e_{k}\right)_{|\phi|}, \bar{e}_{j} \wedge e_{k}>(p) \\
& -\sum_{j, k=1}^{n} \delta_{j}(k) \frac{\left|\lambda_{j}\right|}{\left|\lambda_{j}\right|+\left|\lambda_{k}\right|} \operatorname{Re}\left(\left(Q e_{j} \mid e_{j}\right) \mid\left(\partial M_{\phi} e_{k} \mid e_{k}\right)\right)_{|\phi|}(p) \\
& \left.+\frac{1}{2}\left|\sum_{j=1}^{n}\left(Q e_{j} \mid e_{j}\right)\right|_{|\phi|}^{2}(p)\right)
\end{aligned}
$$

where for $j, k, s=1, \ldots, n$,

$$
a_{j, k, s}(p)=\frac{\delta_{k}(j) \delta_{k}(s)\left|\lambda_{s}(p)\right|}{2\left(\left|\lambda_{j}(p)\right|+\left|\lambda_{k}(p)\right|\right)^{2}\left(\left|\lambda_{j}(p)\right|+\left|\lambda_{k}(p)\right|+\left|\lambda_{s}(p)\right|\right)^{2}},
$$

Remark 1.4. It is straight forward to see that the right side of (1.28) is real (see (5.20) ) and is independent of the choices of eigenvectors $U_{1}, \ldots, U_{n}$.

\section{The Taylor Expansion of $\psi(z, w)$ at $z=w$}

From now on, we assume that $q=n_{-}$. The goal of this work is to compute $\operatorname{Tr} b_{1}(p, p)$, for $p \in \mathbb{C}^{n}$. We may assume that $p=0$ and by taking unitary transformation, we can assume that near 0 ,

$$
\phi(z)=a_{0}+\sum_{j=1}^{n}\left(a_{j} z_{j}+\bar{a}_{j} \bar{z}_{j}\right)+\sum_{j=1}^{n} \lambda_{j}\left|z_{j}\right|^{2}+O\left(|z|^{3}\right),
$$

where $\lambda_{1}, \ldots, \lambda_{n}$ are eigenvalues of $M_{\phi}, a_{0} \in \mathbb{R}, a_{j} \in \mathbb{C}, j=1, \ldots, n$. Put $\widetilde{\phi}=\phi-a_{0}-\sum_{j=1}^{n}\left(a_{j} z_{j}+\bar{a}_{j} \bar{z}_{j}\right)$ and let $\widetilde{\Pi}_{k}^{(q)}$ be the Bergman projection with respect to $e^{-2 k \widetilde{\phi}}$ as in (1.9). It is easy to see that

$$
\Pi_{k}^{(q)}=e^{k a_{0}+k \sum_{j=1}^{n} a_{j} z_{j}} \circ \widetilde{\Pi}_{k}^{(q)} \circ e^{-k a_{0}-k \sum_{j=1}^{n} a_{j} z_{j}} .
$$

Thus, the coefficients of the asymptotic expansion of the kernel of $\Pi_{k}^{(q)}$ on the diagonal are the same as the coefficients of the asymptotic expansion of the kernel of $\widetilde{\Pi}_{k}^{(q)}$.

From the discussion above, we may assume that

$$
\phi(z)=\lambda_{1}\left|z_{1}\right|^{2}+\lambda_{2}\left|z_{2}\right|^{2}+\cdots+\lambda_{n}\left|z_{n}\right|^{2}+O\left(|z|^{3}\right)
$$


near $z=0$. Suppose that $\lambda_{j}<0, j=1, \ldots, q$, and $\lambda_{j}>0, j=q+1, \ldots, n$. In this section, we are going to compute the Taylor expansion of $\psi(z, w)$ at $z=w=0$. We introduce some notations. For $\alpha=\left(\alpha_{1}, \ldots, \alpha_{n}\right), \alpha_{j} \in$ $\mathbb{N} \cup 0, j=1, \ldots, n$, we put $\alpha^{\prime}=\left(\alpha_{1}, \ldots, \alpha_{q}\right), \alpha^{\prime \prime}=\left(\alpha_{q+1}, \ldots, \alpha_{n}\right)$. We write $<\lambda^{\prime}, \alpha^{\prime}>:=\sum_{j=1}^{q} \lambda_{j} \alpha_{j},<\lambda^{\prime \prime}, \alpha^{\prime \prime}>:=\sum_{j=q+1}^{n} \lambda_{j} \alpha_{j},<\left|\lambda^{\prime}\right|, \alpha^{\prime}>:=$ $\sum_{j=1}^{q}\left|\lambda_{j}\right| \alpha_{j}$ and $<\left|\lambda^{\prime \prime}\right|, \alpha^{\prime \prime}>:=\sum_{j=q+1}^{n}\left|\lambda_{j}\right| \alpha_{j}$. The main goal of this section is to prove the following

Theorem 2.1. Under the assumptions and notations before, we have

$$
\begin{aligned}
\psi(z, 0)= & i \sum_{j=1}^{n}\left|\lambda_{j}\right|\left|z_{j}\right|^{2} \\
& +i \sum_{|\alpha|+|\beta|=3,\left(\alpha^{\prime \prime}, \beta^{\prime}\right) \neq 0} \frac{\left\langle\lambda^{\prime \prime}, \alpha^{\prime \prime}>+<\lambda^{\prime}, \beta^{\prime}>\right.}{<\left|\lambda^{\prime \prime}\right|, \alpha^{\prime \prime}>+<\left|\lambda^{\prime}\right|, \beta^{\prime}>} \frac{\partial^{3} \phi}{\partial \bar{z}^{\alpha} \partial z^{\beta}}(0) \frac{\bar{z}^{\alpha} z^{\beta}}{\alpha ! \beta !} \\
& +\frac{i}{2} \sum_{q+1 \leq j, k \leq n, 1 \leq s \leq q} \frac{1}{\left|\lambda_{j}\right|+\left|\lambda_{k}\right|+\left|\lambda_{s}\right|}\left(-\left|\lambda_{j}\right|-\left|\lambda_{k}\right|-\left|\lambda_{s}\right|\right. \\
& \left.+\frac{2\left|\lambda_{j}\right|\left|\lambda_{k}\right|}{\left|\lambda_{j}\right|+\left|\lambda_{s}\right|}+\frac{2\left|\lambda_{j}\right|\left|\lambda_{k}\right|}{\left|\lambda_{k}\right|+\left|\lambda_{s}\right|}\right) \frac{\partial^{3} \phi}{\partial z_{j} \partial z_{k} \partial \bar{z}_{s}}(0) z_{j} z_{k} \bar{z}_{s} \\
& +\frac{i}{2} \sum_{q+1 \leq j \leq n, 1 \leq t, s \leq q} \frac{1}{\left|\lambda_{j}\right|+\left|\lambda_{t}\right|+\left|\lambda_{s}\right|}\left(\left|\lambda_{j}\right|+\left|\lambda_{t}\right|+\left|\lambda_{s}\right|\right. \\
& \left.-\frac{2\left|\lambda_{t}\right|\left|\lambda_{s}\right|}{\left|\lambda_{j}\right|+\left|\lambda_{t}\right|}-\frac{2\left|\lambda_{t}\right|\left|\lambda_{s}\right|}{\left|\lambda_{j}\right|+\left|\lambda_{s}\right|}\right) \frac{\partial^{3} \phi}{\partial z_{j} \partial \bar{z}_{t} \partial \bar{z}_{s}}(0) z_{j} \bar{z}_{t} \bar{z}_{s} \\
& -\frac{i}{3} \sum_{q+1 \leq j, k, s \leq n} \frac{\partial^{3} \phi}{\partial z_{j} \partial z_{k} \partial z_{s}}(0) z_{j} z_{k} z_{s} \\
& +\frac{i}{3} \sum_{1 \leq j, k, s \leq q} \frac{\partial^{3} \phi}{\partial \bar{z}_{j} \partial \bar{z}_{k} \partial \bar{z}_{s}}(0) \bar{z}_{j} \bar{z}_{k} \bar{z}_{s}+O\left(|z|^{4}\right),
\end{aligned}
$$

in some neighborhood of 0 . Moreover, we have

$$
\begin{aligned}
& \frac{\partial^{4} \psi}{\partial \bar{z}_{j} \partial z_{j} \partial \bar{z}_{k} \partial z_{k}}(0,0) \\
& =\sum_{t=1}^{q}\left(\frac{2 i\left|\lambda_{j}\right|}{\left(\left|\lambda_{j}\right|+\left|\lambda_{k}\right|\right)\left(\left|\lambda_{t}\right|+\left|\lambda_{j}\right|\right)}\left|\frac{\partial^{3} \phi}{\partial \bar{z}_{t} \partial z_{j} \partial z_{k}}(0)\right|^{2}\right. \\
& \quad+\frac{2 i\left|\lambda_{t}\right|\left|\lambda_{k}\right|\left|\lambda_{j}\right|}{\left(\left|\lambda_{j}\right|+\left|\lambda_{k}\right|\right)\left(\left|\lambda_{t}\right|+\left|\lambda_{k}\right|+\left|\lambda_{j}\right|\right)^{2}}\left(\frac{1}{\left|\lambda_{j}\right|+\left|\lambda_{t}\right|}+\frac{1}{\left|\lambda_{j}\right|+\left|\lambda_{k}\right|}\right)\left|\frac{\partial^{3} \phi}{\partial \bar{z}_{t} \partial z_{j} \partial \bar{z}_{k}}(0)\right|^{2}
\end{aligned}
$$




$$
\begin{aligned}
& \left.+\frac{2 i\left|\lambda_{j}\right|}{\left(\left|\lambda_{j}\right|+\left|\lambda_{t}\right|\right)\left(\left|\lambda_{j}\right|+\left|\lambda_{k}\right|\right)} \frac{\partial^{3} \phi}{\partial z_{t} \partial \bar{z}_{j} \partial z_{j}}(0) \frac{\partial^{3} \phi}{\partial \bar{z}_{t} \partial \bar{z}_{k} \partial z_{k}}(0)\right) \\
& +\sum_{t=q+1}^{n}\left(\frac{2 i\left|\lambda_{k}\right|}{\left(\left|\lambda_{j}\right|+\left|\lambda_{k}\right|\right)\left(\left|\lambda_{t}\right|+\left|\lambda_{k}\right|\right)}\left|\frac{\partial^{3} \phi}{\partial \bar{z}_{t} \partial z_{j} \partial z_{k}}(0)\right|^{2}\right. \\
& +\frac{2 i\left|\lambda_{t}\right|\left|\lambda_{k}\right|\left|\lambda_{j}\right|}{\left(\left|\lambda_{j}\right|+\left|\lambda_{k}\right|\right)\left(\left|\lambda_{t}\right|+\left|\lambda_{k}\right|+\left|\lambda_{j}\right|\right)^{2}}\left(\frac{1}{\left|\lambda_{j}\right|+\left|\lambda_{k}\right|}+\frac{1}{\left|\lambda_{t}\right|+\left|\lambda_{k}\right|}\right)\left|\frac{\partial^{3} \phi}{\partial \bar{z}_{t} \partial \bar{z}_{j} \partial z_{k}}(0)\right|^{2} \\
& \left.+\frac{2 i\left|\lambda_{k}\right|}{\left(\left|\lambda_{k}\right|+\left|\lambda_{t}\right|\right)\left(\left|\lambda_{j}\right|+\left|\lambda_{k}\right|\right)} \frac{\partial^{3} \phi}{\partial z_{t} \partial \bar{z}_{j} \partial z_{j}}(0) \frac{\partial^{3} \phi}{\partial \bar{z}_{t} \partial \bar{z}_{k} \partial z_{k}}(0)\right) \\
& +i \frac{\lambda_{j}+\lambda_{k}}{\left|\lambda_{j}\right|+\left|\lambda_{k}\right|} \frac{\partial^{4} \phi}{\partial \bar{z}_{j} \partial z_{j} \partial \bar{z}_{k} \partial z_{k}}(0),
\end{aligned}
$$

where $q+1 \leq j \leq n, 1 \leq k \leq q$,

$$
\begin{aligned}
\frac{\partial^{4} \psi}{\partial \bar{z}_{j} \partial z_{j} \partial \bar{z}_{k} \partial z_{k}}(0,0)= & \sum_{t=1}^{q}\left(\frac { 2 i } { ( | \lambda _ { t } | + | \lambda _ { j } | + | \lambda _ { k } | ) ^ { 2 } } \left(\left|\lambda_{t}\right|+\left|\lambda_{j}\right|+\left|\lambda_{k}\right|\right.\right. \\
& \left.\left.-\frac{\left|\lambda_{j}\right|\left|\lambda_{k}\right|}{\left|\lambda_{j}\right|+\left|\lambda_{t}\right|}-\frac{\left|\lambda_{j}\right|\left|\lambda_{k}\right|}{\left|\lambda_{k}\right|+\left|\lambda_{t}\right|}\right)\left|\frac{\partial^{3} \phi}{\partial z_{t} \partial \bar{z}_{j} \partial \bar{z}_{k}}(0)\right|^{2}\right) \\
& +i \frac{\partial^{4} \phi}{\partial \bar{z}_{j} \partial z_{j} \partial \bar{z}_{k} \partial z_{k}}(0),
\end{aligned}
$$

where $q+1 \leq j, k \leq n$, and

$$
\begin{aligned}
\frac{\partial^{4} \psi}{\partial \bar{z}_{j} \partial z_{j} \partial \bar{z}_{k} \partial z_{k}}(0,0)= & \sum_{t=q+1}^{n}\left(\frac { 2 i } { ( | \lambda _ { t } | + | \lambda _ { j } | + | \lambda _ { k } | ) ^ { 2 } } \left(\left|\lambda_{t}\right|+\left|\lambda_{j}\right|+\left|\lambda_{k}\right|\right.\right. \\
& \left.\left.-\frac{\left|\lambda_{j}\right|\left|\lambda_{k}\right|}{\left|\lambda_{k}\right|+\left|\lambda_{t}\right|}-\frac{\left|\lambda_{j}\right|\left|\lambda_{k}\right|}{\left|\lambda_{j}\right|+\left|\lambda_{t}\right|}\right)\left|\frac{\partial^{3} \phi}{\partial \bar{z}_{t} \partial z_{j} \partial z_{k}}(0)\right|^{2}\right) \\
& -i \frac{\partial^{4} \phi}{\partial \bar{z}_{j} \partial z_{j} \partial \bar{z}_{k} \partial z_{k}}(0)
\end{aligned}
$$

where $1 \leq j, k \leq q$.

To prove Theorem 2.1, we first need the following (see [7], for a proof).

Proposition 2.2. Under the assumptions above, we have

$$
\psi(z, w)=i \sum_{j=1}^{n}\left|\lambda_{j}\right|\left|z_{j}-w_{j}\right|^{2}+i \sum_{j=1}^{n} \lambda_{j}\left(\bar{z}_{j} w_{j}-\bar{w}_{j} z_{j}\right)+O\left(|(z, w)|^{3}\right)
$$


near $z=w=0$.

From (2.1) and (2.6), we may write

$$
\begin{gathered}
\psi(z, w)=\quad \\
\quad \sum_{j=1}^{n}\left|\lambda_{j}\right|\left|z_{j}-w_{j}\right|^{2}+i \sum_{j=1}^{n} \lambda_{j}\left(\bar{z}_{j} w_{j}-\bar{w}_{j} z_{j}\right) \\
+\psi_{3}(z, w)+\psi_{4}(z, w)+\cdots
\end{gathered}
$$

where $\psi_{j}(z, w)$ is a homogeneous polynomial of degree $j$ in $(z, w), j=$ $3,4, \ldots$, and

$$
\phi(z)=\sum_{j=1}^{n} \lambda_{j}\left|z_{j}\right|^{2}+\phi_{3}(z)+\phi_{4}(z)+\cdots,
$$

where $\phi_{j}(z)$ is a homogeneous polynomial of degree $j$ in $z, j=3,4, \ldots$. Now, using (2.7) and (2.8) in (1.13), we get

$$
\begin{aligned}
\sum_{j=1}^{q}( & \left(2 \lambda_{j}\left(z_{j}-w_{j}\right)+i \frac{\partial \psi_{3}}{\partial \bar{z}_{j}}+\frac{\partial \phi_{3}}{\partial \bar{z}_{j}}+i \frac{\partial \psi_{4}}{\partial \bar{z}_{j}}+\frac{\partial \phi_{4}}{\partial \bar{z}_{j}}\right) \\
& \left.\times\left(-i \frac{\partial \psi_{3}}{\partial z_{j}}+\frac{\partial \phi_{3}}{\partial z_{j}}-i \frac{\partial \psi_{4}}{\partial z_{j}}+\frac{\partial \phi_{4}}{\partial z_{j}}\right)\right) \\
& +\sum_{j=q+1}^{n}\left(\left(i \frac{\partial \psi_{3}}{\partial \bar{z}_{j}}+\frac{\partial \phi_{3}}{\partial \bar{z}_{j}}+i \frac{\partial \psi_{4}}{\partial \bar{z}_{j}}+\frac{\partial \phi_{4}}{\partial \bar{z}_{j}}\right)\right. \\
& \left.\times\left(2 \lambda_{j}\left(\bar{z}_{j}-\bar{w}_{j}\right)-i \frac{\partial \psi_{3}}{\partial z_{j}}+\frac{\partial \phi_{3}}{\partial z_{j}}-i \frac{\partial \psi_{4}}{\partial z_{j}}+\frac{\partial \phi_{4}}{\partial z_{j}}\right)\right)=O\left(|(z, w)|^{5}\right)
\end{aligned}
$$

We regroup the terms in (2.9) according to the order. Then, the order three and order four terms are the following

$$
\begin{gathered}
T \psi_{3}(z, w)-\sum_{j=1}^{q} 2 i\left|\lambda_{j}\right| w_{j} \frac{\partial \psi_{3}(z, w)}{\partial z_{j}}-\sum_{j=q+1}^{n} 2 i\left|\lambda_{j}\right| \bar{w}_{j} \frac{\partial \psi_{3}(z, w)}{\partial \bar{z}_{j}} \\
=-\sum_{j=1}^{q} 2 \lambda_{j}\left(z_{j}-w_{j}\right) \frac{\partial \phi_{3}(z)}{\partial z_{j}}-\sum_{j=q+1}^{n} 2 \lambda_{j}\left(\bar{z}_{j}-\bar{w}_{j}\right) \frac{\partial \phi_{3}(z)}{\partial \bar{z}_{j}} \\
T \psi_{4}(z, w)-\sum_{j=1}^{q} 2 i\left|\lambda_{j}\right| w_{j} \frac{\partial \psi_{4}(z, w)}{\partial z_{j}}-\sum_{j=q+1}^{n} 2 i\left|\lambda_{j}\right| \bar{w}_{j} \frac{\partial \psi_{4}(z, w)}{\partial \bar{z}_{j}}
\end{gathered}
$$




$$
\begin{aligned}
= & -\sum_{j=1}^{q} 2 \lambda_{j}\left(z_{j}-w_{j}\right) \frac{\partial \phi_{4}(z)}{\partial z_{j}}-\sum_{j=q+1}^{n} 2 \lambda_{j}\left(\bar{z}_{j}-\bar{w}_{j}\right) \frac{\partial \phi_{4}(z)}{\partial \bar{z}_{j}} \\
& -\sum_{j=1}^{n}\left(i \frac{\partial \psi_{3}(z, w)}{\partial \bar{z}_{j}}+\frac{\partial \phi_{3}(z)}{\partial \bar{z}_{j}}\right)\left(-i \frac{\partial \psi_{3}(z, w)}{\partial z_{j}}+\frac{\partial \phi_{3}(z)}{\partial z_{j}}\right),
\end{aligned}
$$

where

$$
T=\sum_{j=1}^{q} 2 i\left|\lambda_{j}\right| z_{j} \frac{\partial}{\partial z_{j}}+\sum_{j=q+1}^{n} 2 i\left|\lambda_{j}\right| \bar{z}_{j} \frac{\partial}{\partial \bar{z}_{j}} .
$$

Let

$$
\psi_{3}=\psi_{3}^{0}+\psi_{3}^{1}+\psi_{3}^{2}+\psi_{3}^{3}
$$

where $\psi_{3}^{j}$ is a homogeneous polynomial of degree $j$ in $w, j=0,1,2,3$. Now, we write (2.10) according to the degree of homogenity in $w$, we get

$$
\begin{aligned}
T \psi_{3}^{0} & =-\sum_{j=1}^{q} 2 \lambda_{j} z_{j} \frac{\partial \phi_{3}}{\partial z_{j}}-\sum_{j=q+1}^{n} 2 \lambda_{j} \bar{z}_{j} \frac{\partial \phi_{3}}{\partial \bar{z}_{j}} \\
T \psi_{3}^{1} & =\sum_{j=1}^{q} 2 \lambda_{j} w_{j}\left(-i \frac{\partial \psi_{3}^{0}}{\partial z_{j}}+\frac{\partial \phi_{3}}{\partial z_{j}}\right)+\sum_{j=q+1}^{n} 2 \lambda_{j} \bar{w}_{j}\left(i \frac{\partial \psi_{3}^{0}}{\partial \bar{z}_{j}}+\frac{\partial \phi_{3}}{\partial \bar{z}_{j}}\right), \\
T \psi_{3}^{2} & =\sum_{j=1}^{q} 2 i\left|\lambda_{j}\right| w_{j} \frac{\partial \psi_{3}^{1}}{\partial z_{j}}+\sum_{j=q+1}^{n} 2 i\left|\lambda_{j}\right| \bar{w}_{j} \frac{\partial \psi_{3}^{1}}{\partial \bar{z}_{j}} .
\end{aligned}
$$

We need

Lemma 2.3. We use the same notations as before. Let

$$
g=\sum_{\alpha, \beta, \gamma, \delta} g_{\alpha, \beta, \gamma, \delta} \bar{z}^{\alpha} z^{\beta} \bar{w}^{\gamma} w^{\delta}, h=\sum_{\alpha, \beta, \gamma, \delta} h_{\alpha, \beta, \gamma, \delta} \bar{z}^{\alpha} z^{\beta} \bar{w}^{\gamma} w^{\delta}
$$

where $\alpha=\left(\alpha_{1}, \ldots, \alpha_{n}\right), \alpha_{j} \in \mathbb{N} \bigcup 0, j=1, \ldots, n$, and similar for $\beta, \gamma, \delta$. If $T g=h$, then

$$
\begin{aligned}
& h_{\alpha, \beta, \gamma, \delta}=0 \quad \text { if }\left(\alpha^{\prime \prime}, \beta^{\prime}\right)=0 \\
& g_{\alpha, \beta, \gamma, \delta}=\frac{1}{2 i} \frac{1}{<\left|\lambda^{\prime \prime}\right|, \alpha^{\prime \prime}>+<\left|\lambda^{\prime}\right|, \beta^{\prime}>} h_{\alpha, \beta, \gamma, \delta} \quad \text { if } \quad\left(\alpha^{\prime \prime}, \beta^{\prime}\right) \neq 0 .
\end{aligned}
$$


Proof. From the definition of $T$ (see (2.12) ), we can compute

$$
T\left(g_{\alpha, \beta, \gamma, \delta} \bar{z}^{\alpha} z^{\beta} \bar{w}^{\gamma} w^{\delta}\right)=0
$$

if $\left(\alpha^{\prime \prime}, \beta^{\prime}\right)=0$, and

$$
T\left(g_{\alpha, \beta, \gamma, \delta} \bar{z}^{\alpha} z^{\beta} \bar{w}^{\gamma} w^{\delta}\right)=2 i\left(<\left|\lambda^{\prime \prime}\right|, \alpha^{\prime \prime}>+<\left|\lambda^{\prime}\right|, \beta^{\prime}>\right) \bar{z}^{\alpha} z^{\beta} \bar{w}^{\gamma} w^{\delta} g_{\alpha, \beta, \gamma, \delta}
$$

if $\left(\alpha^{\prime \prime}, \beta^{\prime}\right) \neq 0$. From this, the lemma follows.

From Lemma 2.3, (2.14), (2.15), (2.16) and very complicated computation, we can determine $\psi_{3}^{0}, \psi_{3}^{1}$ and $\psi_{3}^{2}$ modulo terms $r(z, w)=O\left(|(z, w)|^{3}\right)$ with $\frac{\partial^{|\alpha|+|\beta|} r}{\partial \bar{z}^{\alpha} \partial z^{\beta}}=0$ if $\left(\alpha^{\prime \prime}, \beta^{\prime}\right) \neq 0$. Moreover, we have the following(see Section 2 in [10] for the details)

Proposition 2.4. Under the assumptions and notations before, we have

$$
\begin{aligned}
& \psi(z, w)=i \sum_{j=1}^{n}\left|\lambda_{j}\right|\left|z_{j}-w_{j}\right|^{2}+i \sum_{j=1}^{n} \lambda_{j}\left(\bar{z}_{j} w_{j}-\bar{w}_{j} z_{j}\right) \\
& +i \sum_{|\alpha|+|\beta|=3,\left(\alpha^{\prime \prime}, \beta^{\prime}\right) \neq 0} \frac{<\lambda^{\prime \prime}, \alpha^{\prime \prime}>+<\lambda^{\prime}, \beta^{\prime}>}{<\left|\lambda^{\prime \prime}\right|, \alpha^{\prime \prime}>+<\left|\lambda^{\prime}\right|, \beta^{\prime}>} \frac{\partial^{3} \phi}{\partial \bar{z}^{\alpha} \partial z^{\beta}}(0) \frac{\bar{z}^{\alpha} z^{\beta}}{\alpha ! \beta !} \\
& +i \sum_{j=1|\alpha|+|\beta|=2, \alpha^{\prime \prime} \neq 0}\left(\frac{\bar{z}^{\alpha} z^{\beta} w_{j}}{\alpha ! \beta !} \frac{\partial^{3} \phi}{\partial \bar{z}^{\alpha} \partial z^{\beta} \partial z_{j}}(0)\right. \\
& \left.\quad \times \frac{2\left|\lambda_{j}\right|<\left|\lambda^{\prime \prime}\right|, \alpha^{\prime \prime}>}{\left(<\left|\lambda^{\prime \prime}\right|, \alpha^{\prime \prime}>+<\left|\lambda^{\prime}\right|, \beta^{\prime}>\right)\left(<\left|\lambda^{\prime \prime}\right|, \alpha^{\prime \prime}>+<\left|\lambda^{\prime}\right|, \beta^{\prime}>+\left|\lambda_{j}\right|\right)}\right) \\
& -i \sum_{j=q+1|\alpha|+|\beta|=2, \beta^{\prime} \neq 0}^{n} \sum_{\frac{\alpha}{\alpha} \sum^{\alpha} \bar{w}_{j}} \frac{\partial^{3} \phi}{\partial \bar{z}^{\alpha} \partial z^{\beta} \partial \bar{z}_{j}}(0) \\
& \left.\quad \times \frac{2\left|\lambda_{j}\right|<\left|\lambda^{\prime}\right|, \beta^{\prime}>}{\left(<\left|\lambda^{\prime \prime}\right|, \alpha^{\prime \prime}>+<\left|\lambda^{\prime}\right|, \beta^{\prime}>\right)\left(<\left|\lambda^{\prime \prime}\right|, \alpha^{\prime \prime}>+<\left|\lambda^{\prime}\right|, \beta^{\prime}>+\left|\lambda_{j}\right|\right)}\right) \\
& +i \sum_{q+1 \leq k \leq n, 1 \leq j, s \leq q}\left(\bar{z}_{k} w_{j} w_{s} \frac{\partial^{3} \phi}{\partial \bar{z}_{k} \partial z_{j} \partial z_{s}}(0) \frac{\left|\lambda_{j}\right|\left|\lambda_{s}\right|}{\left|\lambda_{k}\right|+\left|\lambda_{j}\right|+\left|\lambda_{s}\right|}\right. \\
& \left.\quad \times\left(\frac{1}{\left|\lambda_{j}\right|+\left|\lambda_{k}\right|}+\frac{1}{\left|\lambda_{k}\right|+\left|\lambda_{s}\right|}\right)\right) \\
& \quad i \sum_{1 \leq k \leq q, q+1 \leq j, s \leq n}\left(z_{k} \bar{w}_{j} \bar{w}_{s} \frac{\partial^{3} \phi}{\partial z_{k} \partial \bar{z}_{j} \partial \bar{z}_{s}}(0) \frac{\left|\lambda_{j}\right|\left|\lambda_{s}\right|}{\left|\lambda_{k}\right|+\left|\lambda_{j}\right|+\left|\lambda_{s}\right|}\right.
\end{aligned}
$$




$$
\begin{aligned}
& \left.\times\left(\frac{1}{\left|\lambda_{j}\right|+\left|\lambda_{k}\right|}+\frac{1}{\left|\lambda_{k}\right|+\left|\lambda_{s}\right|}\right)\right) \\
& +i \sum_{q+1 \leq s, k \leq n, 1 \leq j \leq q} \bar{z}_{s} \bar{w}_{k} w_{j} \frac{2\left|\lambda_{j}\right|\left|\lambda_{k}\right|}{\left(\left|\lambda_{s}\right|+\left|\lambda_{j}\right|\right)\left(\left|\lambda_{s}\right|+\left|\lambda_{j}\right|+\left|\lambda_{k}\right|\right)} \frac{\partial^{3} \phi}{\partial \bar{z}_{s} \partial z_{j} \partial \bar{z}_{k}}(0) \\
& -i \sum_{q+1 \leq k \leq n, 1 \leq j, s \leq q} z_{s} \bar{w}_{k} w_{j} \frac{2\left|\lambda_{j}\right|\left|\lambda_{k}\right|}{\left(\left|\lambda_{s}\right|+\left|\lambda_{k}\right|\right)\left(\left|\lambda_{s}\right|+\left|\lambda_{j}\right|+\left|\lambda_{k}\right|\right)} \frac{\partial^{3} \phi}{\partial z_{s} \partial z_{j} \partial \bar{z}_{k}}(0) \\
& +R(z, w)+O\left(|(z, w)|^{4}\right),
\end{aligned}
$$

where $R(z, w)=O\left(|(z, w)|^{3}\right)$ and $\frac{\partial^{|\alpha|+|\beta|} R}{\partial \bar{z}^{\alpha} \partial z^{\beta}}=0$ if $\left(\alpha^{\prime \prime}, \beta^{\prime}\right) \neq 0$.

Now, we are ready to compute $\frac{\partial^{3} \psi}{\partial \bar{z}^{\alpha} \partial z^{\beta}}(0,0)$, where $|\alpha|+|\beta|=3$ and $\left(\alpha^{\prime \prime}, \beta^{\prime}\right)=0$. We compute $\frac{\partial^{3} \psi}{\partial \bar{z}_{s_{0}} \partial z_{j_{0}} \partial z_{k_{0}}}(0,0), 1 \leq s_{0} \leq q, q+1 \leq j_{0}, k_{0} \leq n$. From $\psi(z, w)=-\bar{\psi}(w, z)$, we have

$$
\frac{\partial^{3} \psi(z, w)}{\partial \bar{z}_{s_{0}} \partial z_{j_{0}} \partial z_{k_{0}}}=-\overline{\frac{\partial^{3} \psi(z, w)}{\partial w_{s_{0}} \partial \bar{w}_{j_{0}} \partial \bar{w}_{k_{0}}}}
$$

To compute $\frac{\partial^{3} \psi}{\partial \bar{z}_{s_{0}} \partial z_{j_{0}} \partial z_{k_{0}}}(0,0)$, it is equivalent to compute $\frac{\partial^{3} \psi}{\partial w_{s_{0}} \partial \bar{w}_{j_{0}} \partial \bar{w}_{k_{0}}}(0,0)$. From (1.12), we know that

$$
\frac{\partial \psi(z, z)}{\partial \bar{w}_{j_{0}}}=-i \frac{\partial \phi(z)}{\partial \bar{z}_{j_{0}}}
$$

Differentiate (2.21) with respect to $\bar{z}_{k_{0}}$, we get

$$
\frac{\partial^{2} \psi(z, z)}{\partial \bar{w}_{j_{0}} \partial \bar{z}_{k_{0}}}+\frac{\partial^{2} \psi(z, z)}{\partial \bar{w}_{j_{0}} \partial \bar{w}_{k_{0}}}=-i \frac{\partial^{2} \phi(z)}{\partial \bar{z}_{k_{0}} \partial \bar{z}_{j_{0}}} .
$$

Again, differentiate (2.22) with respect to $z_{s_{0}}$, we get

$$
\begin{aligned}
& \frac{\partial^{3} \psi}{\partial \bar{w}_{j_{0}} \partial \bar{z}_{k_{0}} z_{s_{0}}}(0,0)+\frac{\partial^{3} \psi}{\partial \bar{w}_{j_{0}} \partial \bar{z}_{k_{0}} \partial w_{s_{0}}}(0,0) \\
& +\frac{\partial^{3} \psi}{\partial \bar{w}_{j_{0}} \partial \bar{w}_{k_{0}} \partial z_{s_{0}}}(0,0)+\frac{\partial^{3} \psi}{\partial \bar{w}_{j_{0}} \partial \bar{w}_{k_{0}} \partial w_{s_{0}}}(0,0)=-i \frac{\partial^{3} \phi}{\partial \bar{z}_{k_{0}} \partial \bar{z}_{j_{0}} \partial z_{s_{0}}}(0) .
\end{aligned}
$$

From (2.23), we have

$$
\frac{\partial^{3} \psi}{\partial \bar{w}_{j_{0}} \partial \bar{w}_{k_{0}} \partial w_{s_{0}}}(0,0)=-i \frac{\partial^{3} \phi}{\partial \bar{z}_{k_{0}} \partial \bar{z}_{j_{0}} \partial z_{s_{0}}}(0)-\frac{\partial^{3} \psi}{\partial \bar{w}_{j_{0}} \partial \bar{z}_{k_{0}} z_{s_{0}}}(0,0)
$$




$$
-\frac{\partial^{3} \psi}{\partial \bar{w}_{j_{0}} \partial \bar{z}_{k_{0}} \partial w_{s_{0}}}(0,0)-\frac{\partial^{3} \psi}{\partial \bar{w}_{j_{0}} \partial \bar{w}_{k_{0}} \partial z_{s_{0}}}(0,0) .
$$

In view of (2.19), we see that

$$
\begin{aligned}
\frac{\partial^{3} \psi}{\partial \bar{w}_{j_{0}} \partial \bar{z}_{k_{0}} z_{s_{0}}}(0,0)= & \frac{-2 i\left|\lambda_{j_{0}}\right|\left|\lambda_{s_{0}}\right|}{\left(\left|\lambda_{j_{0}}\right|+\left|\lambda_{k_{0}}\right|+\left|\lambda_{s_{0}}\right|\right)\left(\left|\lambda_{k_{0}}\right|+\left|\lambda_{s_{0}}\right|\right)} \frac{\partial^{3} \phi}{\partial \bar{z}_{k_{0}} \partial \bar{z}_{j_{0}} \partial z_{s_{0}}}(0) \\
\frac{\partial^{3} \psi}{\partial \bar{w}_{j_{0}} \partial \bar{z}_{k_{0}} \partial w_{s_{0}}}(0,0)= & \frac{2 i\left|\lambda_{j_{0}}\right|\left|\lambda_{s_{0}}\right|}{\left(\left|\lambda_{j_{0}}\right|+\left|\lambda_{k_{0}}\right|+\left|\lambda_{s_{0}}\right|\right)\left(\left|\lambda_{k_{0}}\right|+\left|\lambda_{s_{0}}\right|\right)} \frac{\partial^{3} \phi}{\partial \bar{z}_{k_{0}} \partial \bar{z}_{j_{0}} \partial z_{s_{0}}}(0) \\
\frac{\partial^{3} \psi}{\partial \bar{w}_{j_{0}} \partial \bar{w}_{k_{0}} \partial z_{s_{0}}}(0,0)= & \frac{-2 i\left|\lambda_{j_{0}}\right|\left|\lambda_{k_{0}}\right|}{\left|\lambda_{k_{0}}\right|+\left|\lambda_{s_{0}}\right|+\left|\lambda_{j_{0}}\right|} \\
& \times\left(\frac{1}{\left|\lambda_{j_{0}}\right|+\left|\lambda_{s_{0}}\right|}+\frac{1}{\left|\lambda_{k_{0}}\right|+\left|\lambda_{s_{0}}\right|}\right) \frac{\partial^{3} \phi}{\partial \bar{z}_{k_{0}} \partial \bar{z}_{j_{0}} \partial z_{s_{0}}}(0) .
\end{aligned}
$$

Combining this with (2.24), we have

$$
\begin{aligned}
& \frac{\partial^{3} \psi}{\partial \bar{w}_{j_{0}} \partial \bar{w}_{k_{0}} \partial w_{s_{0}}}(0,0) \\
& =\left(-i-\frac{-2 i\left|\lambda_{j_{0}}\right|\left|\lambda_{s_{0}}\right|}{\left(\left|\lambda_{j_{0}}\right|+\left|\lambda_{k_{0}}\right|+\left|\lambda_{s_{0}}\right|\right)\left(\left|\lambda_{k_{0}}\right|+\left|\lambda_{s_{0}}\right|\right)}\right. \\
& \quad-\frac{2 i\left|\lambda_{j_{0}}\right|\left|\lambda_{s_{0}}\right|}{\left(\left|\lambda_{j_{0}}\right|+\left|\lambda_{k_{0}}\right|+\left|\lambda_{s_{0}}\right|\right)\left(\left|\lambda_{k_{0}}\right|+\left|\lambda_{s_{0}}\right|\right)} \\
& \left.\quad-\frac{-2 i\left|\lambda_{j_{0}}\right|\left|\lambda_{k_{0}}\right|}{\left|\lambda_{k_{0}}\right|+\left|\lambda_{s_{0}}\right|+\left|\lambda_{j_{0}}\right|}\left(\frac{1}{\left|\lambda_{j_{0}}\right|+\left|\lambda_{s_{0}}\right|}+\frac{1}{\left|\lambda_{k_{0}}\right|+\left|\lambda_{s_{0}}\right|}\right)\right) \frac{\partial^{3} \phi}{\partial \bar{z}_{k_{0}} \partial \bar{z}_{j_{0}} \partial z_{s_{0}}}(0) \\
& =\frac{i}{\left|\lambda_{j_{0}}\right|+\left|\lambda_{k_{0}}\right|+\left|\lambda_{s_{0}}\right|}\left(-\left|\lambda_{j_{0}}\right|-\left|\lambda_{k_{0}}\right|-\left|\lambda_{s_{0}}\right|\right. \\
& \left.\quad+\frac{2\left|\lambda_{j_{0}}\right|\left|\lambda_{k_{0}}\right|}{\left|\lambda_{j_{0}}\right|+\left|\lambda_{s_{0}}\right|}+\frac{2\left|\lambda_{j_{0}}\right|\left|\lambda_{k_{0}}\right|}{\left|\lambda_{k_{0}}\right|+\left|\lambda_{s_{0}}\right|}\right) \frac{\partial^{3} \phi}{\partial \bar{z}_{k_{0}} \partial \bar{z}_{j_{0}} \partial z_{s_{0}}}(0) .
\end{aligned}
$$

From (2.20), we obtain

$$
\begin{aligned}
\frac{\partial^{3} \psi_{3}}{\partial z_{j_{0}} \partial z_{k_{0}} \partial \bar{z}_{s_{0}}}= & \frac{i}{\left|\lambda_{j_{0}}\right|+\left|\lambda_{k_{0}}\right|+\left|\lambda_{s_{0}}\right|}\left(-\left|\lambda_{j_{0}}\right|-\left|\lambda_{k_{0}}\right|-\left|\lambda_{s_{0}}\right|\right. \\
& \left.+\frac{2\left|\lambda_{j_{0}}\right| \lambda_{k_{0}} \mid}{\left|\lambda_{j_{0}}\right|+\left|\lambda_{s_{0}}\right|}+\frac{2\left|\lambda_{j_{0}}\right|\left|\lambda_{k_{0}}\right|}{\left|\lambda_{k_{0}}\right|+\left|\lambda_{s_{0}}\right|}\right) \frac{\partial^{3} \phi}{\partial z_{j_{0}} \partial z_{k_{0}} \partial \bar{z}_{s_{0}}}(0) .
\end{aligned}
$$

We can repeat the method above several times to determine all the terms $\frac{\partial^{3} \psi_{3}}{\partial \bar{z}^{\alpha} \partial z^{\beta}},\left(\alpha^{\prime \prime}, \beta^{\prime}\right)=0$. The computation is straight forward. We omit the process. We state our result 
Proposition 2.5. Under the assumptions and notations before, we have

$$
\begin{aligned}
\psi(z, 0)= & i \sum_{j=1}^{n}\left|\lambda_{j}\right|\left|z_{j}\right|^{2} \\
& +i \sum_{|\alpha|+|\beta|=3,\left(\alpha^{\prime \prime}, \beta^{\prime}\right) \neq 0} \frac{<\lambda^{\prime \prime}, \alpha^{\prime \prime}>+<\lambda^{\prime}, \beta^{\prime}>}{<\left|\lambda^{\prime \prime}\right|, \alpha^{\prime \prime}>+<\left|\lambda^{\prime}\right|, \beta^{\prime}>} \frac{\partial^{3} \phi}{\partial \bar{z}^{\alpha} \partial z^{\beta}}(0) \frac{\bar{z}^{\alpha} z^{\beta}}{\alpha ! \beta !} \\
& +\frac{i}{2} \sum_{q+1 \leq j, k \leq n, 1 \leq s \leq q} \frac{1}{\left|\lambda_{j}\right|+\left|\lambda_{k}\right|+\left|\lambda_{s}\right|}\left(-\left|\lambda_{j}\right|-\left|\lambda_{k}\right|-\left|\lambda_{s}\right|\right. \\
& \left.+\frac{2\left|\lambda_{j}\right|\left|\lambda_{k}\right|}{\left|\lambda_{j}\right|+\left|\lambda_{s}\right|}+\frac{2\left|\lambda_{j}\right|\left|\lambda_{k}\right|}{\left|\lambda_{k}\right|+\left|\lambda_{s}\right|}\right) \frac{\partial^{3} \phi}{\partial z_{j} \partial z_{k} \partial \bar{z}_{s}}(0) z_{j} z_{k} \bar{z}_{s} \\
& +\frac{i}{2} \sum_{q+1 \leq j \leq n, 1 \leq k, s \leq q} \frac{1}{\left|\lambda_{j}\right|+\left|\lambda_{k}\right|+\left|\lambda_{s}\right|}\left(\left|\lambda_{j}\right|+\left|\lambda_{k}\right|+\left|\lambda_{s}\right|\right. \\
& \left.-\frac{2\left|\lambda_{k}\right|\left|\lambda_{s}\right|}{\left|\lambda_{j}\right|+\left|\lambda_{k}\right|}-\frac{2\left|\lambda_{k}\right|\left|\lambda_{s}\right|}{\left|\lambda_{j}\right|+\left|\lambda_{s}\right|}\right) \frac{\partial^{3} \phi}{\partial z_{j} \partial \bar{z}_{k} \partial \bar{z}_{s}}(0) z_{j} \bar{z}_{k} \bar{z}_{s} \\
& -\frac{i}{3} \sum_{q+1 \leq j, k, s \leq n} \frac{\partial^{3} \phi}{\partial z_{j} \partial z_{k} \partial z_{s}}(0) z_{j} z_{k} z_{s} \\
+ & \frac{i}{3} \sum_{1 \leq j, k, s \leq q} \frac{\partial^{3} \phi}{\partial \bar{z}_{j} \partial \bar{z}_{k} \partial \bar{z}_{s}}(0) \bar{z}_{j} \bar{z}_{k} \bar{z}_{s}+O\left(|z|^{4}\right),
\end{aligned}
$$

in some neighborhood of 0 .

Now, to complete the proof of Theorem 2.1, we only need to compute the terms $\frac{\partial^{4} \psi}{\partial \bar{z}_{j} \partial z_{j} \partial \bar{z}_{k} \partial z_{k}}(0,0), 1 \leq j, k \leq n$. Take $w=0$ in (2.11), we obtain

$$
\begin{aligned}
T \psi_{4}(z, 0)= & -\sum_{j=1}^{n}\left(i \frac{\partial \psi_{3}}{\partial \bar{z}_{j}}(z, 0)+\frac{\partial \phi_{3}}{\partial \bar{z}_{j}}(z)\right)\left(-i \frac{\partial \psi_{3}}{\partial z_{j}}(z, 0)+\frac{\partial \phi_{3}}{\partial z_{j}}(z)\right) \\
& -\sum_{j=1}^{q} 2 \lambda_{j} z_{j} \frac{\partial \phi_{4}}{\partial z_{j}}(z)-\sum_{j=q+1}^{n} 2 \lambda_{j} \bar{z}_{j} \frac{\partial \phi_{4}}{\partial \bar{z}_{j}}(z) .
\end{aligned}
$$

From (2.28) and by some direct but very complicated computation, we can determine the terms $\frac{\partial^{4} \psi}{\partial \bar{z}_{j} \partial z_{j} \partial \bar{z}_{k} \partial z_{k}}(0,0), 1 \leq j, k \leq n$ and hence finish the proof of Theorem 2.1. We refer the reader to Section 2 in [10] for the details. 


\section{The Transport Equations for $\square_{k}^{(q)}$}

\subsection{The transport equations}

In this section, we will write down the transport equations for $\square_{k}^{(q)}$ and we will solve the first transport equation at $z=w$ in some sence. The main reference for this section is [1]. We first derive representations for $\bar{\partial}$, $\bar{\partial}_{k}^{*}$ in spaces without exponential weights, by using the following unitary identifications:

$$
\left\{\begin{aligned}
L_{q}^{2}\left(\mathbb{C}^{n}\right) & \leftrightarrow L_{q, k}^{2}\left(\mathbb{C}^{n}\right) \\
u & \leftrightarrow \hat{u}=e^{k \phi} u .
\end{aligned}\right.
$$

Using (3.1), we get

$$
\bar{\partial} \hat{u}=e^{k \phi} \bar{\partial}_{s} u
$$

where

$$
\bar{\partial}_{s}=\sum_{j=1}^{n}\left(d \bar{z}_{j}^{\wedge} \circ\left(\frac{\partial}{\partial \bar{z}_{j}}+k \frac{\partial \phi}{\partial \bar{z}_{j}}\right)\right) .
$$

Now the formal adjoint of $\bar{\partial}_{s}$ for the inner product $(\mid)$ given by (1.4) is

$$
\bar{\partial}_{s}^{*}=\sum_{j=1}^{n}\left(d \bar{z}_{j}^{\wedge, *} \circ\left(-\frac{\partial}{\partial z_{j}}+k \frac{\partial \phi}{\partial z_{j}}\right)\right),
$$

where in view of the unitarity of the relation (3.1),

$$
\bar{\partial}^{*} \hat{u}=e^{k \phi} \bar{\partial}_{s}^{*} u
$$

We can identify the Kodaira Laplacian with

$$
\hat{\square}_{k}^{(q)}=\bar{\partial}_{s} \bar{\partial}_{s}^{*}+\bar{\partial}_{s}^{*} \bar{\partial}_{s}
$$

Put

$$
\hat{\Pi}_{k}^{(q)}: L_{q}^{2}\left(\mathbb{C}^{n}\right) \rightarrow \operatorname{Ker} \hat{\square}_{k}^{(q)}
$$

be the orthogonal projection with respect to $(\mid)$ and let $\hat{\Pi}_{k}^{(q)}(z, w)$ be the distribution kernel of $\hat{\Pi}_{k}^{(q)}$. From (3.2) and (3.5), we have

$$
\hat{\Pi}_{k}^{(q)}(z, w)=e^{-k \phi(z)} \Pi_{k}^{(q)}(z, w) e^{k \phi(w)} .
$$


In view of Theorem 1.1, we see that

$$
\hat{\Pi}_{k}^{(q)}(z, w)=e^{i k \psi(z, w)} b(z, w, k)+S(z, w, k)
$$

where $b(z . w, k) \sim \sum_{o}^{\infty} b_{j}(z, w) k^{n-j}$ in $C^{\infty}\left(\mathbb{C}^{n} \times \mathbb{C}^{n} ; \mathscr{L}\left(\Lambda^{0, q} T_{w}^{*}\left(\mathbb{C}^{n}\right), \Lambda^{0, q} T_{z}^{*}\left(\mathbb{C}^{n}\right)\right)\right)$ and $S(z, w, k)$ is negligible.

From (3.3) and (3.9), we have

$$
e^{-i k \psi} \bar{\partial}_{s} \hat{\Pi}_{k}^{(q)}(z, w)=\sum_{j=1}^{n} k\left(i \frac{\partial \psi}{\partial \bar{z}_{j}}+\frac{\partial \phi}{\partial \bar{z}_{j}}\right) d \bar{z}_{j}^{\wedge} b+\sum_{j=1}^{n} d \bar{z}_{j}^{\wedge} \frac{\partial b}{\partial \bar{z}_{j}} .
$$

From this and (3.4), we can compute

$$
\begin{aligned}
& e^{-i k \psi} \bar{\partial}_{s}^{*} \bar{\partial}_{s} \hat{\Pi}_{k}^{(q)}(z, w)=k^{2} \sum_{j, t=1}^{n}\left(-i \frac{\partial \psi}{\partial z_{t}}+\frac{\partial \phi}{\partial z_{t}}\right)\left(i \frac{\partial \psi}{\partial \bar{z}_{j}}+\frac{\partial \phi}{\partial \bar{z}_{j}}\right) d \bar{z}_{t}^{\wedge, *} d \bar{z}_{j}^{\wedge} b \\
& -k \sum_{j, t=1}^{n} \frac{\partial}{\partial z_{t}}\left(i \frac{\partial \psi}{\partial \bar{z}_{j}}+\frac{\partial \phi}{\partial \bar{z}_{j}}\right) d \bar{z}_{t}^{\wedge, *} d \bar{z}_{j}^{\wedge} b-k \sum_{j, t=1}^{n}\left(i \frac{\partial \psi}{\partial \bar{z}_{j}}+\frac{\partial \phi}{\partial \bar{z}_{j}}\right) d \bar{z}_{t}^{\wedge, *} d \bar{z}_{j}^{\wedge} \frac{\partial b}{\partial z_{t}} \\
& +k \sum_{j, t=1}^{n}\left(-i \frac{\partial \psi}{\partial z_{t}}+\frac{\partial \phi}{\partial z_{t}}\right) d \bar{z}_{t}^{\wedge, *} d \bar{z}_{j}^{\wedge} \frac{\partial b}{\partial \bar{z}_{j}}-\sum_{j, t=1}^{n} d \bar{z}_{t}^{\wedge, *} d \bar{z}_{j}^{\wedge} \frac{\partial^{2} b}{\partial z_{t} \partial \bar{z}_{j}} .
\end{aligned}
$$

Similarly, we have

$$
\begin{aligned}
& e^{-i k \psi} \bar{\partial}_{s} \bar{\partial}_{s}^{*} \hat{\Pi}_{k}^{(q)}(z, w)=k^{2} \sum_{j, t=1}^{n}\left(i \frac{\partial \psi}{\partial \bar{z}_{j}}+\frac{\partial \phi}{\partial \bar{z}_{j}}\right)\left(-i \frac{\partial \psi}{\partial z_{t}}+\frac{\partial \phi}{\partial z_{t}}\right) d \bar{z}_{j}^{\wedge} d \bar{z}_{t}^{\wedge, *} b \\
& +k \sum_{j, t=1}^{n} \frac{\partial}{\partial \bar{z}_{j}}\left(-i \frac{\partial \psi}{\partial z_{t}}+\frac{\partial \phi}{\partial z_{t}}\right) d \bar{z}_{j}^{\wedge} d \bar{z}_{t}^{\wedge, *} b+k \sum_{j, t=1}^{n}\left(-i \frac{\partial \psi}{\partial z_{t}}+\frac{\partial \phi}{\partial z_{t}}\right) d \bar{z}_{j}^{\wedge} d \bar{z}_{t}^{\wedge, *} \frac{\partial b}{\partial \bar{z}_{j}} \\
& -k \sum_{j, t=1}^{n}\left(i \frac{\partial \psi}{\partial \bar{z}_{j}}+\frac{\partial \phi}{\partial \bar{z}_{j}}\right) d \bar{z}_{j}^{\wedge} d \bar{z}_{t}^{\wedge, *} \frac{\partial b}{\partial z_{t}}-\sum_{j, t=1}^{n} d \bar{z}_{j}^{\wedge} d \bar{z}_{t}^{\wedge, *} \frac{\partial^{2} b}{\partial z_{t} \partial \bar{z}_{j}} .
\end{aligned}
$$

Combining (3.11), (3.12) with $d \bar{z}_{j}^{\wedge} \circ d \bar{z}_{k}^{\wedge, *}+d \bar{z}_{k}^{\wedge, *} \circ d \bar{z}_{j}^{\wedge}=\delta_{j, k}$, we get

$$
\begin{aligned}
& e^{-i k \psi} \hat{\square}_{k}^{(q)} \hat{\Pi}_{k}^{(q)}(z, w)=k^{2} \sum_{j=1}^{n}\left(i \frac{\partial \psi}{\partial \bar{z}_{j}}+\frac{\partial \phi}{\partial \bar{z}_{j}}\right)\left(-i \frac{\partial \psi}{\partial z_{j}}+\frac{\partial \phi}{\partial z_{j}}\right) b \\
& +k \sum_{j, t=1}^{n} \frac{\partial}{\partial \bar{z}_{j}}\left(-i \frac{\partial \psi}{\partial z_{t}}+\frac{\partial \phi}{\partial z_{t}}\right) d \bar{z}_{j}^{\wedge} d \bar{z}_{t}^{\wedge, *} b-k \sum_{j, t=1}^{n} \frac{\partial}{\partial z_{t}}\left(i \frac{\partial \psi}{\partial \bar{z}_{j}}+\frac{\partial \phi}{\partial \bar{z}_{j}}\right) d \bar{z}_{t}^{\wedge, *} d \bar{z}_{j}^{\wedge} b
\end{aligned}
$$




$$
+k \sum_{j=1}^{n}\left(-i \frac{\partial \psi}{\partial z_{j}}+\frac{\partial \phi}{\partial z_{j}}\right) \frac{\partial b}{\partial \bar{z}_{j}}-k \sum_{j=1}^{n}\left(i \frac{\partial \psi}{\partial \bar{z}_{j}}+\frac{\partial \phi}{\partial \bar{z}_{j}}\right) \frac{\partial b}{\partial z_{j}}-\sum_{j=1}^{n} \frac{\partial^{2} b}{\partial z_{j} \partial \bar{z}_{j}}
$$

We regroup (3.13) according to the degree of $k$ and notice that the leading term in (3.13) vanishes to infinite order on $z=w$ and $\hat{\square}_{k}^{(q)} \hat{\Pi}_{k}^{(q)}=0$, we obtain the following

Proposition 3.1. We have

$$
\begin{aligned}
& \sum_{j, t=1}^{n} \frac{\partial}{\partial \bar{z}_{j}}\left(-i \frac{\partial \psi}{\partial z_{t}}+\frac{\partial \phi}{\partial z_{t}}\right) d \bar{z}_{j}^{\wedge} d \bar{z}_{t}^{\wedge, *} b_{0}-\sum_{j, t=1}^{n} \frac{\partial}{\partial z_{t}}\left(i \frac{\partial \psi}{\partial \bar{z}_{j}}+\frac{\partial \phi}{\partial \bar{z}_{j}}\right) d \bar{z}_{t}^{\wedge, *} d \bar{z}_{j}^{\wedge} b_{0} \\
& +\sum_{j=1}^{n}\left(-i \frac{\partial \psi}{\partial z_{j}}+\frac{\partial \phi}{\partial z_{j}}\right) \frac{\partial b_{0}}{\partial \bar{z}_{j}}-\sum_{j=1}^{n}\left(i \frac{\partial \psi}{\partial \bar{z}_{j}}+\frac{\partial \phi}{\partial \bar{z}_{j}}\right) \frac{\partial b_{0}}{\partial z_{j}}
\end{aligned}
$$

vanishes to infinite order on $z=w$ and

$$
\begin{aligned}
& \sum_{j, t=1}^{n} \frac{\partial}{\partial \bar{z}_{j}}\left(-i \frac{\partial \psi}{\partial z_{t}}+\frac{\partial \phi}{\partial z_{t}}\right) d \bar{z}_{j}^{\wedge} d \bar{z}_{t}^{\wedge, *} b_{1}-\sum_{j, t=1}^{n} \frac{\partial}{\partial z_{t}}\left(i \frac{\partial \psi}{\partial \bar{z}_{j}}+\frac{\partial \phi}{\partial \bar{z}_{j}}\right) d \bar{z}_{t}^{\wedge, *} d \bar{z}_{j}^{\wedge} b_{1} \\
& +\sum_{j=1}^{n}\left(-i \frac{\partial \psi}{\partial z_{j}}+\frac{\partial \phi}{\partial z_{j}}\right) \frac{\partial b_{1}}{\partial \bar{z}_{j}}-\sum_{j=1}^{n}\left(i \frac{\partial \psi}{\partial \bar{z}_{j}}+\frac{\partial \phi}{\partial \bar{z}_{j}}\right) \frac{\partial b_{1}}{\partial z_{j}}-\sum_{j=1}^{n} \frac{\partial^{2} b_{0}}{\partial z_{j} \partial \bar{z}_{j}}
\end{aligned}
$$

vanishes to infinite order on $z=w$.

\subsection{The first order of the Taylor expansion of $b_{0}(z, w)$ at $z=w$}

Now, as in section 2, we assume that $\phi(z)=\sum_{j=1}^{n} \lambda_{j}\left|z_{j}\right|^{2}+O\left(|z|^{3}\right)$ near 0 and that $\lambda_{j}<0, j=1, \ldots, q$ and $\lambda_{j}>0, j=q+1, \ldots, n$. We work in some neighborhood of $(0,0)$. Put

$$
b_{0}(z, w)=b_{0}^{0}(z, w)+b_{0}^{1}(z, w)+b_{0}^{2}(z, w)+\cdots,
$$

where $b_{0}^{j}$ is a homogeneous polynomialof degree $j$ in $(z, w)$. We recall that

$$
b_{0}^{0}=\pi^{-n}\left|\lambda_{1}\right|\left|\lambda_{2}\right| \cdots\left|\lambda_{n}\right| \prod_{j=1}^{q} d \bar{z}_{j}^{\wedge} d \bar{z}_{j}^{\wedge, *}
$$

(See Theorem 1.2.) For $1 \leq s \leq n$, put $\chi_{1}(s)=1$ if $1 \leq s \leq q$ and $\chi_{1}(s)=0$ if $q+1 \leq s \leq n$ and put $\chi_{2}(s)=1-\chi_{1}(s)$. We recall that for any operator 
$T \in \mathscr{L}\left(\Lambda^{p, q} T^{*}\left(\mathbb{C}^{n}\right), \Lambda^{p, q} T^{*}\left(\mathbb{C}^{n}\right)\right)$, the trace of $T$ is given by (1.1). The first goal of this section is to prove the following

Theorem 3.2. Under the notations above, we have

$$
\begin{aligned}
& b_{0}^{1}(z, 0)= \\
& \sum_{q+1 \leq j \leq n, 1 \leq k \leq q, 1 \leq s \leq n} \frac{1}{\left|\lambda_{j}\right|+\left|\lambda_{k}\right|+\left|\lambda_{s}\right| \chi_{1}(s)} \frac{\partial^{3} \phi}{\partial \bar{z}_{j} \partial z_{k} \partial z_{s}}(0) z_{s} d \bar{z}_{k}^{\wedge, *} d \bar{z}_{j}^{\wedge} b_{0}^{0} \\
& +\sum_{q+1 \leq j \leq n, 1 \leq k \leq q, 1 \leq s \leq n} \frac{1}{\left|\lambda_{j}\right|+\left|\lambda_{k}\right|+\left|\lambda_{s}\right| \chi_{2}(s)} \frac{\partial^{3} \phi}{\partial \bar{z}_{j} \partial z_{k} \partial \bar{z}_{s}}(0) \bar{z}_{s} d \bar{z}_{k}^{\wedge, *} d \bar{z}_{j}^{\wedge} b_{0}^{0} \\
& +\sum_{q+1 \leq j \leq n, 1 \leq k \leq q, q+1 \leq s \leq n} \frac{\left|\lambda_{s}\right|}{\left(\left|\lambda_{j}\right|+\left|\lambda_{k}\right|\right)\left(\left|\lambda_{j}\right|+\left|\lambda_{k}\right|+\left|\lambda_{s}\right|\right)} \frac{\partial^{3} \phi}{\partial z_{j} \partial \bar{z}_{k} \partial z_{s}}(0) z_{s} b_{0}^{0} d \bar{z}_{j}^{\wedge, *} d \bar{z}_{k}^{\wedge} \\
& +\sum_{q+1 \leq j \leq n, 1 \leq k \leq q, 1 \leq s \leq q} \frac{\left|\lambda_{s}\right|}{\left(\left|\lambda_{j}\right|+\left|\lambda_{k}\right|\right)\left(\left|\lambda_{j}\right|+\left|\lambda_{k}\right|+\left|\lambda_{s}\right|\right)} \frac{\partial^{3} \phi}{\partial z_{j} \partial \bar{z}_{k} \partial \bar{z}_{s}}(0) \bar{z}_{s} b_{0}^{0} d \bar{z}_{j}^{\wedge, *} d \bar{z}_{k}^{\wedge} \\
& -\sum_{q+1 \leq s \leq n, 1 \leq j \leq q} \frac{1}{\left|\lambda_{j}\right|+\left|\lambda_{s}\right|} \frac{\partial^{3} \phi}{\partial \bar{z}_{j} \partial z_{j} \partial \bar{z}_{s}}(0) \bar{z}_{s} b_{0}^{0}-\sum_{1 \leq s, j \leq q} \frac{1}{\left|\lambda_{j}\right|} \frac{\partial^{3} \phi}{\partial \bar{z}_{j} \partial z_{j} \partial \bar{z}_{s}}(0) \bar{z}_{s} b_{0}^{0} \\
& +\sum_{1 \leq s \leq q, q+1 \leq j \leq n} \frac{\left|\lambda_{s}\right|}{\left|\lambda_{j}\right|\left(\left|\lambda_{j}\right|+\left|\lambda_{s}\right|\right)} \frac{\partial^{3} \phi}{\partial \bar{z}_{j} \partial z_{j} \partial \bar{z}_{s}}(0) \bar{z}_{s} b_{0}^{0} \\
& +\sum_{1 \leq s \leq q, q+1 \leq j \leq n} \frac{1}{\left|\lambda_{j}\right|+\left|\lambda_{s}\right|} \frac{\partial^{3} \phi}{\partial \bar{z}_{j} \partial z_{j} \partial z_{s}}(0) z_{s} b_{0}^{0} \\
& -\sum_{q+1 \leq s \leq n, 1 \leq j \leq q} \frac{\left|\lambda_{s}\right|}{\left|\lambda_{j}\right|\left(\left|\lambda_{j}\right|+\left|\lambda_{s}\right|\right)} \frac{\partial^{3} \phi}{\partial \bar{z}_{j} \partial z_{j} \partial z_{s}}(0) z_{s} b_{0}^{0} \\
& +\sum_{q+1 \leq s, j \leq n} \frac{1}{\left|\lambda_{j}\right|} \frac{\partial^{3} \phi}{\partial \bar{z}_{j} \partial z_{j} \partial z_{s}}(0) z_{s} b_{0}^{0} \text {. }
\end{aligned}
$$

As in section 2, we write

$$
\begin{aligned}
\psi(z, w)= & i \sum_{j=1}^{n}\left|\lambda_{j}\right|\left|z_{j}-w_{j}\right|^{2}+i \sum_{j=1}^{n} \lambda_{j}\left(\bar{z}_{j} w_{j}-\bar{w}_{j} z_{j}\right) \\
& +\psi_{3}(z, w)+\psi_{4}(z, w)+\cdots
\end{aligned}
$$

and

$$
\phi(z)=\sum_{j=1}^{n} \lambda_{j}\left|z_{j}\right|^{2}+\phi_{3}(z)+\phi_{4}(z)+\cdots
$$


where $\psi_{j}(z, w)$ is a homogeneous polynomial of degree $j$ in $(z, w), j=$ $3,4, \ldots, \phi_{j}(z)$ is a homogeneous polynomial of degree $j$ in $z, j=3,4, \ldots$ Now, using (3.16), (3.18) and (3.19) in (3.14), we get

$$
\begin{aligned}
& \left(\sum_{j=q+1}^{n} 2\left|\lambda_{j}\right| d \bar{z}_{j}^{\wedge} d \bar{z}_{j}^{\wedge, *}+\sum_{j, t=1}^{n} \frac{\partial}{\partial \bar{z}_{j}}\left(-i \frac{\partial \psi_{3}(z, w)}{\partial z_{t}}+\frac{\partial \phi_{3}(z)}{\partial z_{t}}\right) d \bar{z}_{j}^{\wedge} d \bar{z}_{t}^{\wedge, *}\right. \\
& \left.+\sum_{j, t=1}^{n} \frac{\partial}{\partial \bar{z}_{j}}\left(-i \frac{\partial \psi_{4}(z, w)}{\partial z_{t}}+\frac{\partial \phi_{4}(z)}{\partial z_{t}}\right) d \bar{z}_{j}^{\wedge} d \bar{z}_{t}^{\wedge, *}\right)\left(b_{0}^{0}+b_{0}^{1}(z, w)+b_{0}^{2}(z, w)\right) \\
& +\left(\sum_{j=1}^{q} 2\left|\lambda_{j}\right| d \bar{z}_{j}^{\wedge, *} d \bar{z}_{j}^{\wedge}-\sum_{j, t=1}^{n} \frac{\partial}{\partial z_{t}}\left(i \frac{\partial \psi_{3}(z, w)}{\partial \bar{z}_{j}}+\frac{\partial \phi_{3}(z)}{\partial \bar{z}_{j}}\right) d \bar{z}_{t}^{\wedge, *} d \bar{z}_{j}^{\wedge}\right. \\
& \left.-\sum_{j, t=1}^{n} \frac{\partial}{\partial z_{t}}\left(i \frac{\partial \psi_{4}(z, w)}{\partial \bar{z}_{j}}+\frac{\partial \phi_{4}(z)}{\partial \bar{z}_{j}}\right) d \bar{z}_{t}^{\wedge, *} d \bar{z}_{j}^{\wedge}\right)\left(b_{0}^{0}+b_{0}^{1}(z, w)+b_{0}^{2}(z, w)\right) \\
& +\sum_{j=q+1}^{n} 2\left|\lambda_{j}\right|\left(\bar{z}_{j}-\bar{w}_{j}\right)\left(\frac{\partial b_{0}^{1}(z, w)}{\partial \bar{z}_{j}}+\frac{\partial b_{0}^{2}(z, w)}{\partial \bar{z}_{j}}\right)+\sum_{j=1}^{n}\left(-i \frac{\partial \psi_{3}}{\partial z_{j}}+\frac{\partial \phi_{3}}{\partial z_{j}}\right) \frac{\partial b_{0}^{1}(z, w)}{\partial \bar{z}_{j}} \\
& +\sum_{j=1}^{q} 2\left|\lambda_{j}\right|\left(z_{j}-w_{j}\right)\left(\frac{\partial b_{0}^{1}(z, w)}{\partial z_{j}}+\frac{\partial b_{0}^{2}(z, w)}{\partial z_{j}}\right)-\sum_{j=1}^{n}\left(i \frac{\partial \psi_{3}}{\partial \bar{z}_{j}}+\frac{\partial \phi_{3}}{\partial \bar{z}_{j}}\right) \frac{\partial b_{0}^{1}(z, w)}{\partial z_{j}} \\
& =O\left(|(z, w)|^{3}\right) .
\end{aligned}
$$

It is straight forward to see that the order 1 and 2 terms in (3.20) are the following

$$
\begin{aligned}
L b_{0}^{1}(z, w)= & -\left(\sum_{j, t=1}^{n} \frac{\partial}{\partial \bar{z}_{j}}\left(-i \frac{\partial \psi_{3}(z, w)}{\partial z_{t}}+\frac{\partial \phi_{3}(z)}{\partial z_{t}}\right) d \bar{z}_{j}^{\wedge} d \bar{z}_{t}^{\wedge, *}\right. \\
& \left.-\sum_{j, t=1}^{n} \frac{\partial}{\partial z_{t}}\left(i \frac{\partial \psi_{3}(z, w)}{\partial \bar{z}_{j}}+\frac{\partial \phi_{3}(z)}{\partial \bar{z}_{j}}\right) d \bar{z}_{t}^{\wedge, *} d \bar{z}_{j}^{\wedge}\right) b_{0}^{0} \\
& +\sum_{j=q+1}^{n} 2\left|\lambda_{j}\right| \bar{w}_{j} \frac{\partial b_{0}^{1}(z, w)}{\partial \bar{z}_{j}}+\sum_{j=1}^{q} 2\left|\lambda_{j}\right| w_{j} \frac{\partial b_{0}^{1}(z, w)}{\partial z_{j}}
\end{aligned}
$$

and

$$
\begin{aligned}
L b_{0}^{2}(z, w)= & -\left(\sum_{j, t=1}^{n} \frac{\partial}{\partial \bar{z}_{j}}\left(-i \frac{\partial \psi_{4}(z, w)}{\partial z_{t}}+\frac{\partial \phi_{4}(z)}{\partial z_{t}}\right) d \bar{z}_{j}^{\wedge} d \bar{z}_{t}^{\wedge, *}\right. \\
& \left.-\sum_{j, t=1}^{n} \frac{\partial}{\partial z_{t}}\left(i \frac{\partial \psi_{4}(z, w)}{\partial \bar{z}_{j}}+\frac{\partial \phi_{4}(z)}{\partial \bar{z}_{j}}\right) d \bar{z}_{t}^{\wedge, *} d \bar{z}_{j}^{\wedge}\right) b_{0}^{0}
\end{aligned}
$$




$$
\begin{aligned}
& -\left(\sum_{j, t=1}^{n} \frac{\partial}{\partial \bar{z}_{j}}\left(-i \frac{\partial \psi_{3}(z, w)}{\partial z_{t}}+\frac{\partial \phi_{3}(z)}{\partial z_{t}}\right) d \bar{z}_{j}^{\wedge} d \bar{z}_{t}^{\wedge, *}\right. \\
& \left.-\sum_{j, t=1}^{n} \frac{\partial}{\partial z_{t}}\left(i \frac{\partial \psi_{3}(z, w)}{\partial \bar{z}_{j}}+\frac{\partial \phi_{3}(z)}{\partial \bar{z}_{j}}\right) d \bar{z}_{t}^{\wedge, *} d \bar{z}_{j}^{\wedge}\right) b_{0}^{1}(z, w) \\
& -\sum_{j=1}^{n}\left(-i \frac{\partial \psi_{3}(z, w)}{\partial z_{j}}+\frac{\partial \phi_{3}(z)}{\partial z_{j}}\right) \frac{\partial b_{0}^{1}(z, w)}{\partial \bar{z}_{j}} \\
& +\sum_{j=1}^{n}\left(i \frac{\partial \psi_{3}(z, w)}{\partial \bar{z}_{j}}+\frac{\partial \phi_{3}(z)}{\partial \bar{z}_{j}}\right) \frac{\partial b_{0}^{1}(z, w)}{\partial z_{j}} \\
& +\sum_{j=q+1}^{n} 2\left|\lambda_{j}\right| \bar{w}_{j} \frac{\partial b_{0}^{2}(z, w)}{\partial \bar{z}_{j}}+\sum_{j=1}^{q} 2\left|\lambda_{j}\right| w_{j} \frac{\partial b_{0}^{2}(z, w)}{\partial z_{j}}
\end{aligned}
$$

where

$$
\begin{aligned}
L= & \sum_{j=q+1}^{n} 2\left|\lambda_{j}\right| d \bar{z}_{j}^{\wedge} d \bar{z}_{j}^{\wedge, *}+\sum_{j=1}^{q} 2\left|\lambda_{j}\right| d \bar{z}_{j}^{\wedge, *} d \bar{z}_{j}^{\wedge}+\sum_{j=q+1}^{n} 2\left|\lambda_{j}\right| \bar{z}_{j} \frac{\partial}{\partial \bar{z}_{j}} \\
& +\sum_{j=1}^{q} 2\left|\lambda_{j}\right| z_{j} \frac{\partial}{\partial z_{j}} .
\end{aligned}
$$

We rewrite first term of the right side of (3.21):

$$
\begin{gathered}
-\left(\sum_{j, t=1}^{n} \frac{\partial}{\partial \bar{z}_{j}}\left(-i \frac{\partial \psi_{3}(z, w)}{\partial z_{t}}+\frac{\partial \phi_{3}(z)}{\partial z_{t}}\right) d \bar{z}_{j}^{\wedge} d \bar{z}_{t}^{\wedge, *}\right. \\
\left.-\sum_{j, t=1}^{n} \frac{\partial}{\partial z_{t}}\left(i \frac{\partial \psi_{3}(z, w)}{\partial \bar{z}_{j}}+\frac{\partial \phi_{3}(z)}{\partial \bar{z}_{j}}\right) d \bar{z}_{t}^{\wedge, *} d \bar{z}_{j}^{\wedge}\right) b_{0}^{0} \\
=-\sum_{j=1}^{n} \frac{\partial}{\partial \bar{z}_{j}}\left(-i \frac{\partial \psi_{3}(z, w)}{\partial z_{j}}+\frac{\partial \phi_{3}(z)}{\partial z_{j}}\right) d \bar{z}_{j}^{\wedge} d \bar{z}_{j}^{\wedge, *} b_{0}^{0} \\
+\sum_{j=1}^{n} \frac{\partial}{\partial z_{j}}\left(i \frac{\partial \psi_{3}(z, w)}{\partial \bar{z}_{j}}+\frac{\partial \phi_{3}(z)}{\partial \bar{z}_{j}}\right) d \bar{z}_{j}^{\wedge, *} d \bar{z}_{j}^{\wedge} b_{0}^{0} \\
+\sum_{j, t=1, j \neq t}^{n} i \frac{\partial^{2} \psi_{3}(z, w)}{\partial \bar{z}_{j} \partial z_{t}}\left(d \bar{z}_{j}^{\wedge} d \bar{z}_{t}^{\wedge, *}+d \bar{z}_{t}^{\wedge, *} d \bar{z}_{j}^{\wedge}\right) b_{0}^{0} \\
-\sum_{j, t=1 . j \neq t}^{n} \frac{\partial^{2} \phi \phi_{3}(z)}{\partial \bar{z}_{j} \partial z_{t}}\left(d \bar{z}_{j}^{\wedge} d \bar{z}_{t}^{\wedge, *}-d \bar{z}_{t}^{\wedge, *} d \bar{z}_{j}^{\wedge}\right) b_{0}^{0} .
\end{gathered}
$$


Note that $d \bar{z}_{j}^{\wedge} d \bar{z}_{t}^{\wedge, *}+d \bar{z}_{t}^{\wedge, *} d \bar{z}_{j}^{\wedge}=\delta_{j, t}$. From the form of $b_{0}^{0}$ (see Theorem[1.2), we can check that $d \bar{z}_{j}^{\wedge} d \bar{z}_{j}^{\wedge, *} b_{0}^{0}=0$ if $q+1 \leq j \leq n, d \bar{z}_{j}^{\wedge} d \bar{z}_{j}^{\wedge, *} b_{0}^{0}=b_{0}^{0}$ if $1 \leq j \leq q, d \bar{z}_{j}^{\wedge, *} d \bar{z}_{j}^{\wedge} b_{0}^{0}=0$ if $1 \leq j \leq q, d \bar{z}_{j}^{\wedge, *} d \bar{z}_{j}^{\wedge} b_{0}^{0}=b_{0}^{0}$ if $q+1 \leq j \leq n$ and when $j \neq t, d \bar{z}_{t}^{\wedge, *} d \bar{z}_{j}^{\wedge} b_{0}^{0} \neq 0$ if and only if $q+1 \leq j \leq n$ and $1 \leq t \leq q$. From this observaton, (3.24) becomes

$$
\begin{aligned}
& -\left(\sum_{j, t=1}^{n} \frac{\partial}{\partial \bar{z}_{j}}\left(-i \frac{\partial \psi_{3}(z, w)}{\partial z_{t}}+\frac{\partial \phi_{3}(z)}{\partial z_{t}}\right) d \bar{z}_{j}^{\wedge} d \bar{z}_{t}^{\wedge, *}\right. \\
& \left.-\sum_{j, t=1}^{n} \frac{\partial}{\partial z_{t}}\left(i \frac{\partial \psi_{3}(z, w)}{\partial \bar{z}_{j}}+\frac{\partial \phi_{3}(z)}{\partial \bar{z}_{j}}\right) d \bar{z}_{t}^{\wedge, *} d \bar{z}_{j}^{\wedge}\right) b_{0}^{0} \\
& =-\sum_{j=1}^{q} \frac{\partial}{\partial \bar{z}_{j}}\left(-i \frac{\partial \psi_{3}(z, w)}{\partial z_{j}}+\frac{\partial \phi_{3}(z)}{\partial z_{j}}\right) b_{0}^{0}+\sum_{j=q+1}^{n} \frac{\partial}{\partial z_{j}}\left(i \frac{\partial \psi_{3}(z, w)}{\partial \bar{z}_{j}}+\frac{\partial \phi_{3}(z)}{\partial \bar{z}_{j}}\right) b_{0}^{0} \\
& \quad+2 \sum_{q+1 \leq j \leq n, 1 \leq t \leq q}^{n} \frac{\partial^{2} \phi_{3}(z)}{\partial \bar{z}_{j} \partial z_{t}} d \bar{z}_{t}^{\wedge, *} d \bar{z}_{j}^{\wedge} b_{0}^{0} .
\end{aligned}
$$

From (2.19), it is straight forward to see that

$$
\frac{\partial}{\partial \bar{z}_{j}}\left(-i \frac{\partial \psi_{3}(z, w)}{\partial z_{j}}+\frac{\partial \phi_{3}(z)}{\partial z_{j}}\right)=\sum_{q+1 \leq s \leq n} \frac{2\left|\lambda_{s}\right|}{\left|\lambda_{j}\right|+\left|\lambda_{s}\right|} \frac{\partial^{3} \phi}{\partial \bar{z}_{j} \partial z_{j} \partial \bar{z}_{s}}(0)\left(\bar{z}_{s}-\bar{w}_{s}\right)
$$

where $1 \leq j \leq q$ and

$$
\frac{\partial}{\partial z_{j}}\left(i \frac{\partial \psi_{3}(z, w)}{\partial \bar{z}_{j}}+\frac{\partial \phi_{3}(z)}{\partial \bar{z}_{j}}\right)=\sum_{1 \leq s \leq q} \frac{2\left|\lambda_{s}\right|}{\left|\lambda_{j}\right|+\left|\lambda_{s}\right|} \frac{\partial^{3} \phi}{\partial \bar{z}_{j} \partial z_{j} \partial z_{s}}(0)\left(z_{s}-w_{s}\right)
$$

where $q+1 \leq j \leq n$. From (3.25), (3.26) and (3.27), (3.21) becomes

$$
\begin{aligned}
L b_{0}^{1}(z, w)= & -\sum_{q+1 \leq s \leq n, 1 \leq j \leq q} \frac{2\left|\lambda_{s}\right|}{\left|\lambda_{j}\right|+\left|\lambda_{s}\right|} \frac{\partial^{3} \phi}{\partial \bar{z}_{j} \partial z_{j} \partial \bar{z}_{s}}(0)\left(\bar{z}_{s}-\bar{w}_{s}\right) b_{0}^{0} \\
& +\sum_{1 \leq s \leq q, q+1 \leq j \leq n} \frac{2\left|\lambda_{s}\right|}{\left|\lambda_{j}\right|+\left|\lambda_{s}\right|} \frac{\partial^{3} \phi}{\partial \bar{z}_{j} \partial z_{j} \partial z_{s}}(0)\left(z_{s}-w_{s}\right) b_{0}^{0} \\
& +2 \sum_{q+1 \leq j \leq n, 1 \leq t \leq q, 1 \leq s \leq n}^{n} \frac{\partial^{3} \phi}{\partial \bar{z}_{j} \partial z_{t} \partial z_{s}}(0) z_{s} d \bar{z}_{t}^{\wedge, *} d \bar{z}_{j}^{\wedge} b_{0}^{0}
\end{aligned}
$$




$$
\begin{aligned}
& +2 \sum_{q+1 \leq j \leq n, 1 \leq t \leq q, 1 \leq s \leq n}^{n} \frac{\partial^{3} \phi}{\partial \bar{z}_{j} \partial z_{t} \partial \bar{z}_{s}}(0) \bar{z}_{s} d \bar{z}_{t}^{\wedge, *} d \bar{z}_{j}^{\wedge} b_{0}^{0} \\
& +\sum_{j=q+1}^{n} 2\left|\lambda_{j}\right| \bar{w}_{j} \frac{\partial b_{0}^{1}(z, w)}{\partial \bar{z}_{j}}+\sum_{j=1}^{q} 2\left|\lambda_{j}\right| w_{j} \frac{\partial b_{0}^{1}(z, w)}{\partial z_{j}} .
\end{aligned}
$$

Now, we write (3.28) according to the degree of homogenity in $w$, we get

$$
\begin{aligned}
L b_{0}^{1}(z, 0)= & -\sum_{q+1 \leq s \leq n, 1 \leq j \leq q} \frac{2\left|\lambda_{s}\right|}{\left|\lambda_{j}\right|+\left|\lambda_{s}\right|} \frac{\partial^{3} \phi}{\partial \bar{z}_{j} \partial z_{j} \partial \bar{z}_{s}}(0) \bar{z}_{s} b_{0}^{0} \\
& +\sum_{1 \leq s \leq q, q+1 \leq j \leq n} \frac{2\left|\lambda_{s}\right|}{\left|\lambda_{j}\right|+\left|\lambda_{s}\right|} \frac{\partial^{3} \phi}{\partial \bar{z}_{j} \partial z_{j} \partial z_{s}}(0) z_{s} b_{0}^{0} \\
& +2 \sum_{q+1 \leq j \leq n, 1 \leq t \leq q, 1 \leq s \leq n}^{n} \frac{\partial^{3} \phi}{\partial \bar{z}_{j} \partial z_{t} \partial z_{s}}(0) z_{s} d \bar{z}_{t}^{\wedge, *} d \bar{z}_{j}^{\wedge} b_{0}^{0} \\
& +2 \sum_{q+1 \leq j \leq n, 1 \leq t \leq q, 1 \leq s \leq n}^{n} \frac{\partial^{3} \phi}{\partial \bar{z}_{j} \partial z_{t} \partial \bar{z}_{s}}(0) \bar{z}_{s} d \bar{z}_{t}^{\wedge, *} d \bar{z}_{j}^{\wedge} b_{0}^{0}
\end{aligned}
$$

and

$$
\begin{aligned}
L b_{0}^{1}(0, w)= & \sum_{q+1 \leq s \leq n, 1 \leq j \leq q} \frac{2\left|\lambda_{s}\right|}{\left|\lambda_{j}\right|+\left|\lambda_{s}\right|} \frac{\partial^{3} \phi}{\partial \bar{z}_{j} \partial z_{j} \partial \bar{z}_{s}}(0) \bar{w}_{s} b_{0}^{0} \\
& -\sum_{1 \leq s \leq q, q+1 \leq j \leq n} \frac{2\left|\lambda_{s}\right|}{\left|\lambda_{j}\right|+\left|\lambda_{s}\right|} \frac{\partial^{3} \phi}{\partial \bar{z}_{j} \partial z_{j} \partial z_{s}}(0) w_{s} b_{0}^{0} \\
& +\sum_{j=q+1}^{n} 2\left|\lambda_{j}\right| \bar{w}_{j} \frac{\partial b_{0}^{1}(z, 0)}{\partial \bar{z}_{j}}+\sum_{j=1}^{q} 2\left|\lambda_{j}\right| w_{j} \frac{\partial b_{0}^{1}(z, 0)}{\partial z_{j}} .
\end{aligned}
$$

We pause and introduce some notations. For multi-index $J$, we write $|J|=q, J \nearrow$, if $J=\left(j_{1}, \ldots, j_{q}\right), 1 \leq j_{1}<j_{2}<\cdots<j_{q} \leq n$. Set $d \bar{z}^{J}=d \bar{z}_{j_{1}} \wedge \cdots \wedge d \bar{z}_{j_{q}}, J=\left(j_{1}, \ldots, j_{q}\right)$. Then, $d \bar{z}^{J},|J|=q, J \nearrow$, is an orthonormal basis of $\Lambda^{0, q} T_{0}^{*}\left(\mathbb{C}^{n}\right)$, where 0 is the origin in $\mathbb{C}^{n}$. Let $M_{d \bar{z}^{J}, d \bar{z}^{K}}$, $|J|=|K|=q, J, K \nearrow$, be the $\mathbb{C}$-linear operator:

$$
\begin{aligned}
M_{d \bar{z}^{J}, d \bar{z}^{K}}: \Lambda^{0, q} T_{0}^{*}\left(\mathbb{C}^{n}\right) & \rightarrow \Lambda^{0, q} T_{0}^{*}\left(\mathbb{C}^{n}\right) \\
d \bar{z}^{J} & \rightarrow d \bar{z}^{K} \\
d \bar{z}^{I} & \rightarrow 0 \text { if } I \neq J .
\end{aligned}
$$


It is clear that $M_{d \bar{z}^{J}, d \bar{z}^{K}},|J|=|K|=q, J, K \nearrow$, is a basis of the vector space $\mathscr{L}\left(\Lambda^{0, q} T_{0}^{*}\left(\mathbb{C}^{n}\right), \Lambda^{0, q} T_{0}^{*}\left(\mathbb{C}^{n}\right)\right)$. For $m \in \mathbb{N} \bigcup\{0\}$, put

$$
\begin{aligned}
& P^{m}\left(\mathscr{L}\left(\Lambda^{0, q} T_{0}^{*}\left(\mathbb{C}^{n}\right), \Lambda^{0, q} T_{0}^{*}\left(\mathbb{C}^{n}\right)\right)\right) \\
& =\left\{\sum_{|\alpha|+|\beta|+|\gamma|+|\delta|=m} A_{\alpha, \beta} \bar{z}^{\alpha} z^{\beta} \bar{w}^{\gamma} w^{\delta} ; A_{\alpha, \beta} \in \mathscr{L}\left(\Lambda^{0, q} T_{0}^{*}\left(\mathbb{C}^{n}\right), \Lambda^{0, q} T_{0}^{*}\left(\mathbb{C}^{n}\right)\right)\right\} .
\end{aligned}
$$

For multi-index $J,|J|=q$, we define

$$
F(J)=2 \sum_{j \in J, q+1 \leq j \leq n}\left|\lambda_{j}\right|+2 \sum_{j \notin J, 1 \leq j \leq q}\left|\lambda_{j}\right| .
$$

Put $I_{0}=(1, \ldots, q)$. Note that $F(J) \neq 0$ if and only if $J \neq I_{0}$. We have the following

Lemma 3.3. We use the same notations as in the discussion before Theorem 2.1 and before. If we consider $L$ as the operator (We recall that $L$ is given by (3.23).)

$$
L: P^{m}\left(\mathscr{L}\left(\Lambda^{0, q} T_{0}^{*}\left(\mathbb{C}^{n}\right), \Lambda^{0, q} T_{0}^{*}\left(\mathbb{C}^{n}\right)\right)\right) \rightarrow P^{m}\left(\mathscr{L}\left(\Lambda^{0, q} T_{0}^{*}\left(\mathbb{C}^{n}\right), \Lambda^{0, q} T_{0}^{*}\left(\mathbb{C}^{n}\right)\right)\right)
$$

Then,

$\operatorname{Ker} L$

$$
=\left\{\sum_{|\alpha|+|\beta|+|\gamma|+|\delta|=m,\left(\alpha^{\prime \prime}, \beta^{\prime}\right)=0,|J|=q, J \nearrow} c_{J, I_{0}}^{\alpha, \beta, \gamma, \delta} M_{d \bar{z} J, d \bar{z}^{I} \bar{z}^{\alpha}} \bar{z}^{\beta} \bar{w}^{\gamma} w^{\delta} ; c_{J, I_{0}}^{\alpha, \beta, \gamma} \in \mathbb{C}\right\} .
$$

Moreover, for $A \in P^{m}\left(\mathscr{L}\left(\Lambda^{0, q} T_{0}^{*}\left(\mathbb{C}^{n}\right), \Lambda^{0, q} T_{0}^{*}\left(\mathbb{C}^{n}\right)\right)\right)$, we write

$$
A=\sum_{|\alpha|+|\beta|+|\gamma|+|\delta|=m,|J|=|K|=q, J, K \nearrow} c_{J, K}^{\alpha, \beta, \gamma, \delta} M_{d \bar{z}^{J}, d \bar{z}^{K}} \bar{z}^{\alpha} z^{\beta} \bar{w}^{\gamma} w^{\delta},
$$

$c_{J, K}^{\alpha, \beta, \gamma, \delta} \in \mathbb{C}$. If $c_{J, I_{0}}^{\alpha, \beta, \gamma, \delta}=0$ when $\left(\alpha^{\prime \prime}, \beta^{\prime}\right)=0$. Then, we have $L B=A$, where

$$
\begin{aligned}
B= & \sum_{|\alpha|+|\beta|+|\gamma|+|\delta|=m,|J|=|K|=q, J, K \nearrow, K \neq I_{0}} \text { if }\left(\alpha^{\prime \prime}, \beta^{\prime}\right)=0 \\
& \times \frac{1}{F(K)+2<\left|\lambda^{\prime \prime}\right|, \alpha^{\prime \prime}>+2<\left|\lambda^{\prime}\right|, \beta^{\prime}>} \bar{z}^{\alpha} z^{\beta} \bar{w}^{\gamma} w^{\delta}+u(z), \quad \\
& c_{J, K}^{\alpha, \gamma, \delta} M_{d \bar{z}^{J}, d \bar{z}^{K}}
\end{aligned}
$$


where $u \in \operatorname{Ker} L$.

Proof. We recall that

$$
\begin{aligned}
L= & \sum_{j=q+1}^{n} 2\left|\lambda_{j}\right| d \bar{z}_{j}^{\wedge} d \bar{z}_{j}^{\wedge, *}+\sum_{j=1}^{q} 2\left|\lambda_{j}\right| d \bar{z}_{j}^{\wedge, *} d \bar{z}_{j}^{\wedge}+\sum_{j=q+1}^{n} 2\left|\lambda_{j}\right| \bar{z}_{j} \frac{\partial}{\partial \bar{z}_{j}} \\
& +\sum_{j=1}^{q} 2\left|\lambda_{j}\right| z_{j} \frac{\partial}{\partial z_{j}} .
\end{aligned}
$$

For $M_{d \bar{z}^{J}, d \bar{z}^{K}},|J|=|K|=q, J, K \nearrow$, we have

$$
\begin{aligned}
& \left(L\left(M_{d \bar{z}^{J}, d \bar{z}^{K}} \bar{z}^{\alpha} z^{\beta} \bar{w}^{\gamma} w^{\delta}\right)\right) d \bar{z}^{J} \\
& =\left(\sum_{j=q+1}^{n} 2\left|\lambda_{j}\right| d \bar{z}_{j}^{\wedge} d \bar{z}_{j}^{\wedge, *}+\sum_{j=1}^{q} 2\left|\lambda_{j}\right| d \bar{z}_{j}^{\wedge, *} d \bar{z}_{j}^{\wedge}\right. \\
& \left.+\sum_{j=q+1}^{n} 2\left|\lambda_{j}\right| \bar{z}_{j} \frac{\partial}{\partial \bar{z}_{j}}+\sum_{j=1}^{q} 2\left|\lambda_{j}\right| z_{j} \frac{\partial}{\partial z_{j}}\right) d \bar{z}^{K} \bar{z}^{\alpha} z^{\beta} \\
& =\left(2 \sum_{j \in K, q+1 \leq j \leq n}\left|\lambda_{j}\right|+2 \sum_{j \notin K, 1 \leq j \leq q}\left|\lambda_{j}\right|\right. \\
& \left.+2\left(<\left|\lambda^{\prime \prime}\right|, \alpha^{\prime \prime}>+<\left|\lambda^{\prime}\right|, \beta^{\prime}>\right)\right) d \bar{z}^{K} \bar{z}^{\alpha} z^{\beta} \bar{w}^{\gamma} w^{\delta} \\
& =\left(F(K)+2\left(<\left|\lambda^{\prime \prime}\right|, \alpha^{\prime \prime}>+<\left|\lambda^{\prime}\right|, \beta^{\prime}>\right)\right) d \bar{z}^{K} \bar{z}^{\alpha} z^{\beta} \bar{w}^{\gamma} w^{\delta}
\end{aligned}
$$

and

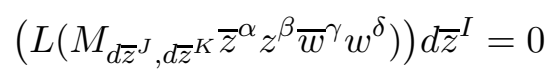

if $I \neq J$. Thus,

$$
\begin{aligned}
& L\left(M_{d \bar{z}^{J}, d \bar{z}^{K}}\right) \bar{z}^{\alpha} z^{\beta} \bar{w}^{\gamma} w^{\delta} \\
& =\left(F(K)+2\left(<\left|\lambda^{\prime \prime}\right|, \alpha^{\prime \prime}>+<\left|\lambda^{\prime}\right|, \beta^{\prime}>\right)\right) M_{d \bar{z}^{J}, d \bar{z}^{K}} \bar{z}^{\alpha} z^{\beta} \bar{w}^{\gamma} w^{\delta} .
\end{aligned}
$$

From (3.35), the lemma follows.

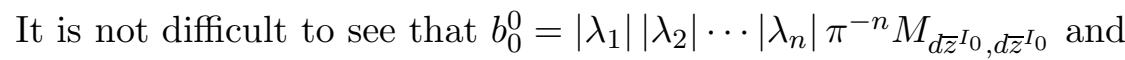

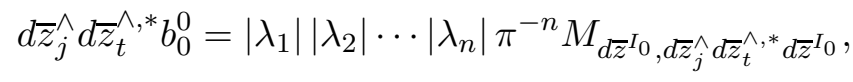

where $q+1 \leq j \leq n, 1 \leq t \leq q$. Combining this with (3.29) and Lemma 3.3. 
we get the following

Proposition 3.4. We have that

$$
\begin{aligned}
b_{0}^{1}(z, 0) & =\sum_{q+1 \leq j \leq n, 1 \leq t \leq q, 1 \leq s \leq n} \frac{1}{\left|\lambda_{j}\right|+\left|\lambda_{t}\right|+\left|\lambda_{s}\right| \chi_{1}(s)} \frac{\partial^{3} \phi}{\partial \bar{z}_{j} \partial z_{t} \partial z_{s}}(0) z_{s} d \bar{z}_{t}^{\wedge, *} d \bar{z}_{j}^{\wedge} b_{0}^{0} \\
& +\sum_{q+1 \leq j \leq n, 1 \leq t \leq q, 1 \leq s \leq n} \frac{1}{\left|\lambda_{j}\right|+\left|\lambda_{t}\right|+\left|\lambda_{s}\right| \chi_{2}(s)} \frac{\partial^{3} \phi}{\partial \bar{z}_{j} \partial z_{t} \partial \bar{z}_{s}}(0) \bar{z}_{s} d \bar{z}_{t}^{\wedge, *} d \bar{z}_{j}^{\wedge} b_{0}^{0} \\
& -\sum_{q+1 \leq s \leq n, 1 \leq j \leq q} \frac{1}{\left|\lambda_{j}\right|+\left|\lambda_{s}\right|} \frac{\partial^{3} \phi}{\partial \bar{z}_{j} \partial z_{j} \partial \bar{z}_{s}}(0) \bar{z}_{s} b_{0}^{0} \\
& +\sum_{1 \leq s \leq q, q+1 \leq j \leq n} \frac{1}{\left|\lambda_{j}\right|+\left|\lambda_{s}\right|} \frac{\partial^{3} \phi}{\partial \bar{z}_{j} \partial z_{j} \partial z_{s}}(0) z_{s} b_{0}^{0}+u(z),
\end{aligned}
$$

where $u(z) \in \operatorname{Ker} L$.

Now, we compute $b_{0}^{1}(0, w)$. From (3.37), we can compute the last two terms of the right side of (3.30):

$$
\begin{aligned}
& \sum_{s=q+1}^{n} 2\left|\lambda_{s}\right| \bar{w}_{s} \frac{\partial b_{0}^{1}(z, 0)}{\partial \bar{z}_{s}}+\sum_{s=1}^{q} 2\left|\lambda_{s}\right| w_{s} \frac{\partial b_{0}^{1}(z, 0)}{\partial z_{s}} \\
& =\sum_{q+1 \leq j \leq n, 1 \leq t \leq q, 1 \leq s \leq q} \frac{2\left|\lambda_{s}\right|}{\left|\lambda_{j}\right|+\left|\lambda_{t}\right|+\left|\lambda_{s}\right|} \frac{\partial^{3} \phi}{\partial \bar{z}_{j} \partial z_{t} \partial z_{s}}(0) w_{s} d \bar{z}_{t}^{\wedge, *} d \bar{z}_{j}^{\wedge} b_{0}^{0} \\
& \quad+\sum_{q+1 \leq j \leq n, 1 \leq t \leq q, q+1 \leq s \leq n} \frac{2\left|\lambda_{s}\right|}{\left|\lambda_{j}\right|+\left|\lambda_{t}\right|+\left|\lambda_{s}\right|} \frac{\partial^{3} \phi}{\partial \bar{z}_{j} \partial z_{t} \partial \bar{z}_{s}}(0) \bar{w}_{s} d \bar{z}_{t}^{\wedge, *} d \bar{z}_{j}^{\wedge} b_{0}^{0} \\
& \quad-\sum_{q+1 \leq s \leq n, 1 \leq j \leq q} \frac{2\left|\lambda_{s}\right|}{\left|\lambda_{j}\right|+\left|\lambda_{s}\right|} \frac{\partial^{3} \phi}{\partial \bar{z}_{j} \partial z_{j} \partial \bar{z}_{s}}(0) \bar{w}_{s} b_{0}^{0} \\
& \quad+\sum_{1 \leq s \leq q, q+1 \leq j \leq n} \frac{2\left|\lambda_{s}\right|}{\left|\lambda_{j}\right|+\left|\lambda_{s}\right|} \frac{\partial^{3} \phi}{\partial \bar{z}_{j} \partial z_{j} \partial z_{s}}(0) w_{s} b_{0}^{0} .
\end{aligned}
$$

Combining this with (3.30), we obtain

$$
\begin{aligned}
& L b_{0}^{1}(0, w)=\sum_{q+1 \leq j \leq n, 1 \leq t \leq q, 1 \leq s \leq q} \frac{2\left|\lambda_{s}\right|}{\left|\lambda_{j}\right|+\left|\lambda_{t}\right|+\left|\lambda_{s}\right|} \frac{\partial^{3} \phi}{\partial \bar{z}_{j} \partial z_{t} \partial z_{s}}(0) w_{s} d \bar{z}_{t}^{\wedge, *} d \bar{z}_{j}^{\wedge} b_{0}^{0} \\
& +\sum_{q+1 \leq j \leq n, 1 \leq t \leq q, q+1 \leq s \leq n} \frac{2\left|\lambda_{s}\right|}{\left|\lambda_{j}\right|+\left|\lambda_{t}\right|+\left|\lambda_{s}\right|} \frac{\partial^{3} \phi}{\partial \bar{z}_{j} \partial z_{t} \partial \bar{z}_{s}}(0) \bar{w}_{s} d \bar{z}_{t}^{\wedge, *} d \bar{z}_{j}^{\wedge} b_{0}^{0} . \quad
\end{aligned}
$$


From this, (3.36) and Lemma 3.3, we get

Proposition 3.5. We have that

$$
\begin{aligned}
& b_{0}^{1}(0, w)=\sum_{q+1 \leq j \leq n, 1 \leq t \leq q, 1 \leq s \leq q} \frac{\left|\lambda_{s}\right|}{\left(\left|\lambda_{j}\right|+\left|\lambda_{t}\right|\right)\left(\left|\lambda_{j}\right|+\left|\lambda_{t}\right|+\left|\lambda_{s}\right|\right)} \\
& \times \frac{\partial^{3} \phi}{\partial \bar{z}_{j} \partial z_{t} \partial z_{s}}(0) w_{s} d \bar{z}_{t}^{\wedge, *} d \bar{z}_{j}^{\wedge} b_{0}^{0} \\
&+\sum_{q+1 \leq j \leq n, 1 \leq t \leq q, q+1 \leq s \leq n} \frac{\left|\lambda_{s}\right|}{\left(\left|\lambda_{j}\right|+\left|\lambda_{t}\right|\right)\left(\left|\lambda_{j}\right|+\left|\lambda_{t}\right|+\left|\lambda_{s}\right|\right)} \\
& \times \frac{\partial^{3} \phi}{\partial \bar{z}_{j} \partial z_{t} \partial \bar{z}_{s}}(0) \bar{w}_{s} d \bar{z}_{t}^{\wedge, *} d \bar{z}_{j}^{\wedge} b_{0}^{0}+v(w),
\end{aligned}
$$

where $v(w) \in \operatorname{Ker} L$.

In view of Proposition 3.4, we know that to prove Theorem 3.2, we only need to compute $u(z)$, where $u(z)$ is as in (3.37). Now, we compute $u(z)$. Note that $u(z) \in \operatorname{Ker} L$. From Lemma 3.3, we may write

$$
\begin{aligned}
& u(z)=\sum_{q+1 \leq s \leq n,|J|=q, J \nearrow, J \neq I_{0}} c_{J, I_{0}}^{s} M_{d \bar{z}^{J}, d \bar{z}^{I_{0}} z_{s}} \\
& +\sum_{1 \leq s \leq q,|J|=q, J \nearrow, J \neq I_{0}} c_{J, I_{0}}^{s} M_{d \bar{z}^{J}, d \bar{z}^{I_{0}}} \bar{z}_{s} \\
& +\sum_{q+1 \leq s \leq n} z_{s} c^{s} M_{d \bar{z}^{I_{0}}, d \bar{z}^{I_{0}}}+\sum_{1 \leq s \leq q} \bar{z}_{s} c^{s} M_{d \bar{z}^{I_{0}, d \bar{z}^{I}}},
\end{aligned}
$$

where $c_{J, I_{0}}^{s}, c^{s} \in \mathbb{C}$, for all $s=1, \ldots, n,|J|=q, J \nearrow, J \neq I_{0}$. Let $u^{*}(z)$ be the adjoint of $u(z)$ with respect to $(\mid)$ in the space $\mathscr{L}\left(\Lambda^{0, q} T^{*}\left(\mathbb{C}^{n}\right), \Lambda^{0, q} T^{*}\left(\mathbb{C}^{n}\right)\right)$. We can check that

$$
\begin{aligned}
& u^{*}(z)=\sum_{q+1 \leq s \leq n,|J|=q, J \nearrow, J \neq I_{0}} \overline{c_{J, I_{0}}^{s}} M_{d \bar{z}^{I_{0}}, d \bar{z}^{J} \bar{z}_{s}} \\
& +\sum_{1 \leq s \leq q,|J|=q, J \nearrow, J \neq I_{0}} \overline{c_{J, I_{0}}^{s}} M_{d \bar{z}^{I_{0}, d \bar{z}^{J}} z_{s}} \\
& +\sum_{1 \leq s \leq q} \overline{c^{s}} z_{s} M_{d \bar{z}^{I_{0}}, d \bar{z}^{I_{0}}}+\sum_{q+1 \leq s \leq n} \overline{c^{s}} \bar{z}_{s} M_{d \bar{z}^{I_{0}}, d \bar{z}^{I_{0}}} .
\end{aligned}
$$

We notice that the Bergman projection $\Pi_{k}^{(q)}$ is self-adjoint. From this obser- 
vation, we deduce that

$$
\left(b_{0}^{1}(w, 0)\right)^{*}=b_{0}^{1}(0, w)
$$

where $\left(b_{0}^{1}(w, 0)\right)^{*}$ is the adjoint of $b_{0}^{1}(w, 0)$ with respect to the inner product ( | ) in the space $\mathscr{L}\left(\Lambda^{0, q} T^{*}\left(\mathbb{C}^{n}\right), \Lambda^{0, q} T^{*}\left(\mathbb{C}^{n}\right)\right)$. From (3.37) and (3.42) and recall that $\operatorname{Ker} L$ is given by (3.33), we deduce that

$$
\begin{aligned}
\left(b_{0}^{1}(w, 0)\right)^{*}= & \sum_{q+1 \leq s \leq n,|J|=q, J \nearrow, J \neq I_{0}} \overline{c_{J, I_{0}}^{s}} M_{d \bar{z}^{I_{0}, d \bar{z}^{J}} \bar{w}_{s}} \overline{c_{J, I_{0}}^{s}} M_{d \bar{z}^{I_{0}}, d \bar{z}^{J}} w_{s}+r(w), \\
& +\sum_{1 \leq s \leq q,|J|=q, J \nearrow, J \neq I_{0}}
\end{aligned}
$$

where $r(w) \in \operatorname{Ker} L$. From (3.40) and (3.36), we have

$$
\begin{aligned}
& b_{0}^{1}(0, w)=\left|\lambda_{1}\right| \cdots\left|\lambda_{n}\right| \pi^{-n} \\
& \times\left(\sum_{q+1 \leq j \leq n, 1 \leq t \leq q, 1 \leq s \leq q} \frac{\left|\lambda_{s}\right|}{\left(\left|\lambda_{j}\right|+\left|\lambda_{t}\right|\right)\left(\left|\lambda_{j}\right|+\left|\lambda_{t}\right|+\left|\lambda_{s}\right|\right)}\right.
\end{aligned}
$$

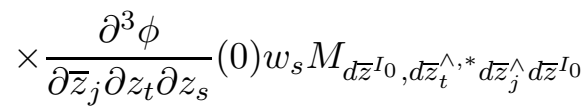

$$
\begin{aligned}
& +\sum_{q+1 \leq j \leq n, 1 \leq t \leq q, q+1 \leq s \leq n} \frac{\left|\lambda_{s}\right|}{\left(\left|\lambda_{j}\right|+\left|\lambda_{t}\right|\right)\left(\left|\lambda_{j}\right|+\left|\lambda_{t}\right|+\left|\lambda_{s}\right|\right)}
\end{aligned}
$$

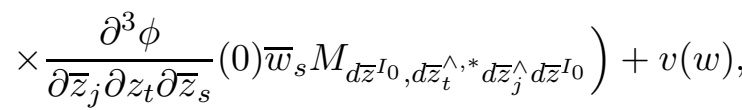

where $v(w) \in \operatorname{Ker} L$. From (3.43), (3.44) and (3.45), we get

$$
\begin{aligned}
& c_{J, I_{0}}^{s}=\left|\lambda_{1}\right| \cdots\left|\lambda_{n}\right| \pi^{-n} \times \frac{\left|\lambda_{s}\right|}{\left(\left|\lambda_{j}\right|+\left|\lambda_{t}\right|\right)\left(\left|\lambda_{j}\right|+\left|\lambda_{t}\right|+\left|\lambda_{s}\right|\right)} \times \frac{\partial^{3} \phi}{\partial z_{j} \partial \bar{z}_{t} \partial \bar{z}_{s}}(0) \\
& \text { if } J=d \bar{z}_{t}^{\wedge, *} d \bar{z}_{j}^{\wedge} d \bar{z}^{I_{0}}, q+1 \leq j \leq n, 1 \leq t \leq q, \text { and } s=1, \ldots, q, \\
& c_{J, I_{0}}^{s}=\left|\lambda_{1}\right| \cdots\left|\lambda_{n}\right| \pi^{-n} \times \frac{\left|\lambda_{s}\right|}{\left(\left|\lambda_{j}\right|+\left|\lambda_{t}\right|\right)\left(\left|\lambda_{j}\right|+\left|\lambda_{t}\right|+\left|\lambda_{s}\right|\right)} \times \frac{\partial^{3} \phi}{\partial z_{j} \partial \bar{z}_{t} \partial z_{s}}(0) \\
& \text { if } J=d \bar{z}_{t}^{\wedge, *} d \bar{z}_{j}^{\wedge} d \bar{z}^{I_{0}}, q+1 \leq j \leq n, 1 \leq t \leq q, \text { and } s=q+1, \ldots, n \\
& c_{J, I_{0}}^{s}=0 \text { otherwise. }
\end{aligned}
$$


Combining above with (3.41) and (3.36), we obtain

$$
\begin{aligned}
& b_{0}^{1}(z, 0)=\sum_{q+1 \leq j \leq n, 1 \leq t \leq q, 1 \leq s \leq n} \frac{1}{\left|\lambda_{j}\right|+\left|\lambda_{t}\right|+\left|\lambda_{s}\right| \chi_{1}(s)} \frac{\partial^{3} \phi}{\partial \bar{z}_{j} \partial z_{t} \partial z_{s}}(0) z_{s} d \bar{z}_{t}^{\wedge, *} d \bar{z}_{j}^{\wedge} b_{0}^{0} \\
& +\sum_{q+1 \leq j \leq n, 1 \leq t \leq q, 1 \leq s \leq n} \frac{1}{\left|\lambda_{j}\right|+\left|\lambda_{t}\right|+\left|\lambda_{s}\right| \chi_{2}(s)} \frac{\partial^{3} \phi}{\partial \bar{z}_{j} \partial z_{t} \partial \bar{z}_{s}}(0) \bar{z}_{s} d \bar{z}_{t}^{\wedge, *} d \bar{z}_{j}^{\wedge} b_{0}^{0} \\
& -\sum_{q+1 \leq s \leq n, 1 \leq j \leq q} \frac{1}{\left|\lambda_{j}\right|+\left|\lambda_{s}\right|} \frac{\partial^{3} \phi}{\partial \bar{z}_{j} \partial z_{j} \partial \bar{z}_{s}}(0) \bar{z}_{s} b_{0}^{0} \\
& +\sum_{1 \leq s \leq q, q+1 \leq j \leq n} \frac{1}{\left|\lambda_{j}\right|+\left|\lambda_{s}\right|} \frac{\partial^{3} \phi}{\partial \bar{z}_{j} \partial z_{j} \partial z_{s}}(0) z_{s} b_{0}^{0} \\
& +\sum_{q+1 \leq j \leq n, 1 \leq t \leq q, q+1 \leq s \leq n} \frac{\left|\lambda_{s}\right|}{\left(\left|\lambda_{j}\right|+\left|\lambda_{t}\right|\right)\left(\left|\lambda_{j}\right|+\left|\lambda_{t}\right|+\left|\lambda_{s}\right|\right)} \\
& \times \frac{\partial^{3} \phi}{\partial z_{j} \partial \bar{z}_{t} \partial z_{s}}(0) z_{s} b_{0}^{0} d \bar{z}_{j}^{\wedge, *} d \bar{z}_{t}^{\wedge} \\
& +\sum_{q+1 \leq j \leq n, 1 \leq t \leq q, 1 \leq s \leq q} \frac{\left|\lambda_{s}\right|}{\left(\left|\lambda_{j}\right|+\left|\lambda_{t}\right|\right)\left(\left|\lambda_{j}\right|+\left|\lambda_{t}\right|+\left|\lambda_{s}\right|\right)} \\
& \times \frac{\partial^{3} \phi}{\partial z_{j} \partial \bar{z}_{t} \partial \bar{z}_{s}}(0) \bar{z}_{s} b_{0}^{0} d \bar{z}_{j}^{\wedge, *} d \bar{z}_{t}^{\wedge} \\
& +\sum_{q+1 \leq s \leq n} z_{s} c^{s} M_{d \bar{z}^{I_{0}}, d \bar{z}^{I_{0}}}+\sum_{1 \leq s \leq q} \bar{z}_{s} c^{s} M_{d \bar{z}^{I_{0}, d \bar{z}^{I_{0}}} .} .
\end{aligned}
$$

Now, to complete the proof of Theorem 3.2, we only need to know $c^{s}, s=$ $1, \ldots, n$. From (3.49), we know that

$$
\begin{aligned}
\operatorname{Tr} b_{0}^{1}(z, 0)= & -\left|\lambda_{1}\right| \cdots\left|\lambda_{n}\right| \pi^{-n}\left(\sum_{q+1 \leq s \leq n, 1 \leq j \leq q} \frac{1}{\left|\lambda_{j}\right|+\left|\lambda_{s}\right|} \frac{\partial^{3} \phi}{\partial \bar{z}_{j} \partial z_{j} \partial \bar{z}_{s}}(0) \bar{z}_{s}\right) \\
& +\left|\lambda_{1}\right| \cdots\left|\lambda_{n}\right| \pi^{-n}\left(\sum_{1 \leq s \leq q, q+1 \leq j \leq n} \frac{1}{\left|\lambda_{j}\right|+\left|\lambda_{s}\right|} \frac{\partial^{3} \phi}{\partial \bar{z}_{j} \partial z_{j} \partial z_{s}}(0) z_{s}\right) \\
& +\sum_{q+1 \leq s \leq n} z_{s} c^{s}+\sum_{1 \leq s \leq q} \bar{z}_{s} c^{s} .
\end{aligned}
$$

From Theorem 1.2, we know that $\operatorname{Tr} b_{0}(z, z)=\pi^{-n}(-1)^{q} \operatorname{det}\left(\frac{\partial^{2} \phi}{\partial \bar{z}_{j} \partial z_{k}}(z)\right)_{j, k=1}^{n}$. 
From this, we can compute

$$
\begin{aligned}
\left.\frac{\partial}{\partial z_{s}} \operatorname{Tr} b_{0}(z, z)\right|_{z=0}= & \left.\pi^{-n}(-1)^{q} \frac{\partial}{\partial z_{s}} \operatorname{det}\left(\frac{\partial^{2} \phi}{\partial \bar{z}_{j} \partial z_{k}}(z)\right)_{j, k=1}^{n}\right|_{z=0} \\
= & \pi^{-n}\left|\lambda_{1}\right| \cdots\left|\lambda_{n}\right|\left(-\sum_{1 \leq j \leq q} \frac{1}{\left|\lambda_{j}\right|} \frac{\partial^{3} \phi}{\partial \bar{z}_{j} \partial z_{j} \partial z_{s}}(0)\right. \\
& \left.+\sum_{q+1 \leq j \leq n} \frac{1}{\left|\lambda_{j}\right|} \frac{\partial^{3} \phi}{\partial \bar{z}_{j} \partial z_{j} \partial z_{s}}(0)\right)
\end{aligned}
$$

From (3.43), we know that $\overline{\operatorname{Tr} b_{0}^{1}(z, 0)}=\operatorname{Tr} b_{0}^{1}(0, z)$. Note that $b_{0}^{1}(z, z)=$ $b_{0}^{1}(z, 0)+b_{0}^{1}(0, z)$. From this, we see that

$$
\begin{aligned}
\left.\frac{\partial}{\partial z_{s}} \operatorname{Tr} b_{0}(z, z)\right|_{z=0} & =\frac{\partial}{\partial z_{s}} \operatorname{Tr} b_{0}^{1}(z, 0)+\frac{\partial}{\partial z_{s}} \operatorname{Tr} b_{0}^{1}(0, z) \\
& =\frac{\partial}{\partial z_{s}} \operatorname{Tr} b_{0}^{1}(z, 0)+\frac{\partial}{\partial \bar{z}_{s}} \operatorname{Tr} b_{0}^{1}(z, 0) .
\end{aligned}
$$

Thus, if $q+1 \leq s \leq n$, from (3.50) and (3.52), we can check that

$$
\begin{aligned}
& \left.\frac{\partial}{\partial z_{s}} \operatorname{Tr} b_{0}(z, z)\right|_{z=0} \\
& =-\left|\lambda_{1}\right| \cdots\left|\lambda_{n}\right| \pi^{-n}\left(\sum_{1 \leq j \leq q} \frac{1}{\left|\lambda_{j}\right|+\left|\lambda_{s}\right|} \frac{\partial^{3} \phi}{\partial \bar{z}_{j} \partial z_{j} \partial z_{s}}(0)\right)+c^{s} .
\end{aligned}
$$

From (3.51) and (3.53), we can compute

$$
\begin{aligned}
c_{s}= & \pi^{-n}\left|\lambda_{1}\right| \cdots\left|\lambda_{n}\right|\left(-\sum_{1 \leq j \leq q} \frac{1}{\left|\lambda_{j}\right|} \frac{\partial^{3} \phi}{\partial \bar{z}_{j} \partial z_{j} \partial z_{s}}(0)+\sum_{q+1 \leq j \leq n} \frac{1}{\left|\lambda_{j}\right|} \frac{\partial^{3} \phi}{\partial \bar{z}_{j} \partial z_{j} \partial z_{s}}(0)\right) \\
& +\pi^{-n}\left|\lambda_{1}\right| \cdots\left|\lambda_{n}\right|\left(\sum_{1 \leq j \leq q} \frac{1}{\left|\lambda_{j}\right|+\left|\lambda_{s}\right|} \frac{\partial^{3} \phi}{\partial \bar{z}_{j} \partial z_{j} \partial z_{s}}(0)\right) \\
= & \pi^{-n}\left|\lambda_{1}\right| \cdots\left|\lambda_{n}\right|\left(-\sum_{1 \leq j \leq q} \frac{\left|\lambda_{s}\right|}{\left|\lambda_{j}\right|\left(\left|\lambda_{j}\right|+\left|\lambda_{s}\right|\right)} \frac{\partial^{3} \phi}{\partial \bar{z}_{j} \partial z_{j} \partial z_{s}}(0)\right. \\
& \left.+\sum_{q+1 \leq j \leq n} \frac{1}{\left|\lambda_{j}\right|} \frac{\partial^{3} \phi}{\partial \bar{z}_{j} \partial z_{j} \partial z_{s}}(0)\right),
\end{aligned}
$$


$q+1 \leq s \leq n$. Similarly, we can repeat the procedure above and get

$$
\begin{aligned}
c_{s}= & \pi^{-n}\left|\lambda_{1}\right| \cdots\left|\lambda_{n}\right|\left(-\sum_{1 \leq j \leq q} \frac{1}{\left|\lambda_{j}\right|} \frac{\partial^{3} \phi}{\partial \bar{z}_{j} \partial z_{j} \partial \bar{z}_{s}}(0)\right. \\
& \left.+\sum_{q+1 \leq j \leq n} \frac{\left|\lambda_{s}\right|}{\left|\lambda_{j}\right|\left(\left|\lambda_{j}\right|+\left|\lambda_{s}\right|\right)} \frac{\partial^{3} \phi}{\partial \bar{z}_{j} \partial z_{j} \partial \bar{z}_{s}}(0)\right)
\end{aligned}
$$

if $1 \leq s \leq q$. Combining (3.55), (3.54) with (3.49), Theorem 3.2 follows.

3.3. The second order of the Taylor expansion of $b_{0}(z, w)$ at $z=w$ and the $b_{1}$ term

The second goal of this section is to prove the following

Theorem 3.6. Put

$$
\operatorname{Tr} b_{0}^{1}(z, 0)=\pi^{-n}\left|\lambda_{1}\right| \cdots\left|\lambda_{n}\right|\left(\sum_{s=1}^{n}\left(a_{s} z_{s}+b_{s} \bar{z}_{s}\right)\right) .
$$

Then for $b_{0}^{2}(z, w)$ in (3.16), we have

$$
\begin{aligned}
& b_{0}^{2}(z, 0) \\
& =\sum_{q+1 \leq j \leq n, 1 \leq k \leq q, 1 \leq s \leq n} \frac{1}{\left|\lambda_{s}\right|\left(\left|\lambda_{j}\right|+\left|\lambda_{k}\right|+\left|\lambda_{s}\right| \chi_{1}(s)\right)}\left|\frac{\partial^{3} \phi}{\partial \bar{z}_{j} \partial z_{k} \partial z_{s}}(0)\right|^{2}\left|z_{s}\right|^{2} b_{0}^{0} \\
& +\sum_{q+1 \leq j \leq n, 1 \leq k \leq q, 1 \leq s \leq n} \frac{1}{\left|\lambda_{s}\right|\left(\left|\lambda_{j}\right|+\left|\lambda_{k}\right|+\left|\lambda_{s}\right| \chi_{2}(s)\right)}\left|\frac{\partial^{3} \phi}{\partial \bar{z}_{j} \partial z_{k} \partial \bar{z}_{s}}(0)\right|^{2}\left|z_{s}\right|^{2} b_{0}^{0} \\
& +\sum_{q+1 \leq j \leq n, 1 \leq k \leq q, q+1 \leq s \leq n} \frac{\left|\lambda_{s}\right|}{\left(\left|\lambda_{j}\right|+\left|\lambda_{k}\right|\right)\left(\left|\lambda_{j}\right|+\left|\lambda_{k}\right|+\left|\lambda_{s}\right|\right)^{2}}\left|\frac{\partial^{3} \phi}{\partial \bar{z}_{j} \partial z_{k} \partial \bar{z}_{s}}(0)\right|^{2} \\
& \times\left|z_{s}\right|^{2} d \bar{z}_{k}^{\wedge, *} d \bar{z}_{j}^{\wedge} b_{0}^{0} d \bar{z}_{j}^{\wedge, *} d \bar{z}_{k}^{\wedge} \\
& +\sum_{q+1 \leq j \leq n, 1 \leq k \leq q, 1 \leq s \leq q} \frac{\left|\lambda_{s}\right|}{\left(\left|\lambda_{j}\right|+\left|\lambda_{k}\right|\right)\left(\left|\lambda_{j}\right|+\left|\lambda_{k}\right|+\left|\lambda_{s}\right|\right)^{2}}\left|\frac{\partial^{3} \phi}{\partial \bar{z}_{j} \partial z_{k} \partial z_{s}}(0)\right|^{2} \\
& \times\left|z_{s}\right|^{2} d \bar{z}_{k}^{\wedge, *} d \bar{z}_{j}^{\wedge} b_{0}^{0} d \bar{z}_{j}^{\wedge, *} d \bar{z}_{k}^{\wedge} \\
& -\sum_{q+1 \leq u \leq n, 1 \leq s \leq n} \frac{1}{\left|\lambda_{u}\right|+\left|\lambda_{s}\right| \chi_{1}(s)} b_{s} \frac{\partial^{3} \phi}{\partial \bar{z}_{u} \partial z_{u} \partial z_{s}}(0)\left|z_{u}\right|^{2} b_{0}^{0}
\end{aligned}
$$




$$
\begin{aligned}
& +\sum_{1 \leq u \leq q, 1 \leq s \leq n} \frac{1}{\left|\lambda_{u}\right|+\left|\lambda_{s}\right| \chi_{2}(s)} a_{s} \frac{\partial^{3} \phi}{\partial \bar{z}_{u} \partial z_{u} \partial \bar{z}_{s}}(0)\left|z_{u}\right|^{2} b_{0}^{0} \\
& -\sum_{1 \leq j \leq q, q+1 \leq s \leq n} \frac{1}{\left|\lambda_{j}\right|+\left|\lambda_{s}\right|} a_{s} \frac{\partial^{3} \phi}{\partial \bar{z}_{j} \partial z_{j} \partial \bar{z}_{s}}(0)\left|z_{s}\right|^{2} b_{0}^{0} \\
& +\sum_{q+1 \leq j \leq n, 1 \leq s \leq q} \frac{1}{\left|\lambda_{j}\right|+\left|\lambda_{s}\right|} b_{s} \frac{\partial^{3} \phi}{\partial \bar{z}_{j} \partial z_{j} \partial z_{s}}(0)\left|z_{s}\right|^{2} b_{0}^{0} \\
& -\sum_{1 \leq j \leq q, 1 \leq k \leq n} \frac{\partial^{4}(-i \psi(z, 0)+\phi)}{\partial \bar{z}_{j} \partial z_{j} \partial \bar{z}_{k} \partial z_{k}}(0) \frac{\left|z_{k}\right|^{2}}{2\left|\lambda_{k}\right|} b_{0}^{0} \\
& +\sum_{q+1 \leq j \leq n, 1 \leq k \leq n} \frac{\partial^{4}(i \psi(z, 0)+\phi)}{\partial \bar{z}_{j} \partial z_{j} \partial \bar{z}_{k} \partial z_{k}}(0) \frac{\left|z_{k}\right|^{2}}{2\left|\lambda_{k}\right|} b_{0}^{0}+r(z)+h(z)
\end{aligned}
$$

where $\operatorname{Tr} h=0$ and $\frac{\partial^{2} r}{\partial \bar{z}_{j} \partial z_{j}}=0, j=1, \ldots, n$.

Furthermore, we have

$$
\begin{aligned}
b_{1}(0,0)= & \sum_{q+1 \leq j \leq n, 1 \leq k \leq q, 1 \leq s \leq q} \frac{\left|\lambda_{s}\right|}{2\left(\left|\lambda_{j}\right|+\left|\lambda_{k}\right|\right)^{2}\left(\left|\lambda_{j}\right|+\left|\lambda_{k}\right|+\left|\lambda_{s}\right|\right)^{2}} \\
& \times\left|\frac{\partial^{3} \phi}{\partial \bar{z}_{j} \partial z_{k} \partial z_{s}}(0)\right|^{2} d \bar{z}_{k}^{\wedge, *} d \bar{z}_{j}^{\wedge} b_{0}^{0} d \bar{z}_{j}^{\wedge, *} d \bar{z}_{k}^{\wedge} \\
& +\sum_{q+1 \leq j \leq n, 1 \leq k \leq q, q+1 \leq s \leq n} \frac{\left|\lambda_{s}\right|}{2\left(\left|\lambda_{j}\right|+\left|\lambda_{k}\right|\right)^{2}\left(\left|\lambda_{j}\right|+\left|\lambda_{k}\right|+\left|\lambda_{s}\right|\right)^{2}} \\
& \times\left|\frac{\partial^{3} \phi}{\partial \bar{z}_{j} \partial z_{k} \partial \bar{z}_{s}}(0)\right|^{2} d \bar{z}_{k}^{\wedge, *} d \bar{z}_{j}^{\wedge} b_{0}^{0} d \bar{z}_{j}^{\wedge, *} d \bar{z}_{k}^{\wedge}+c b_{0}^{0}+R,
\end{aligned}
$$

where $c \in \mathbb{C}, \operatorname{Tr} R=0$.

Take $w=0$ in (3.22), we get

$$
\begin{aligned}
L b_{0}^{2}(z, 0)= & -\left(\sum_{j, t=1}^{n} \frac{\partial}{\partial \bar{z}_{j}}\left(-i \frac{\partial \psi_{4}(z, 0)}{\partial z_{t}}+\frac{\partial \phi_{4}(z)}{\partial z_{t}}\right) d \bar{z}_{j}^{\wedge} d \bar{z}_{t}^{\wedge, *}\right. \\
& \left.-\sum_{j, t=1}^{n} \frac{\partial}{\partial z_{t}}\left(i \frac{\partial \psi_{4}(z, 0)}{\partial \bar{z}_{j}}+\frac{\partial \phi_{4}(z)}{\partial \bar{z}_{j}}\right) d \bar{z}_{t}^{\wedge, *} d \bar{z}_{j}^{\wedge}\right) b_{0}^{0} \\
& -\left(\sum_{j, t=1}^{n} \frac{\partial}{\partial \bar{z}_{j}}\left(-i \frac{\partial \psi_{3}(z, 0)}{\partial z_{t}}+\frac{\partial \phi_{3}(z)}{\partial z_{t}}\right) d \bar{z}_{j}^{\wedge} d \bar{z}_{t}^{\wedge, *}\right.
\end{aligned}
$$




$$
\begin{aligned}
& \left.-\sum_{j, t=1}^{n} \frac{\partial}{\partial z_{t}}\left(i \frac{\partial \psi_{3}(z, 0)}{\partial \bar{z}_{j}}+\frac{\partial \phi_{3}(z)}{\partial \bar{z}_{j}}\right) d \bar{z}_{t}^{\wedge, *} d \bar{z}_{j}^{\wedge}\right) b_{0}^{1}(z, 0) \\
& -\sum_{j=1}^{n}\left(-i \frac{\partial \psi_{3}(z, 0)}{\partial z_{j}}+\frac{\partial \phi_{3}(z)}{\partial z_{j}}\right) \frac{\partial b_{0}^{1}(z, 0)}{\partial \bar{z}_{j}} \\
& +\sum_{j=1}^{n}\left(i \frac{\partial \psi_{3}(z, 0)}{\partial \bar{z}_{j}}+\frac{\partial \phi_{3}(z)}{\partial \bar{z}_{j}}\right) \frac{\partial b_{0}^{1}(z, 0)}{\partial z_{j}}
\end{aligned}
$$

From Lemma 3.3, (3.59) and some very complicated computation (see Section 3.3 in [10] for the details), we get (3.57).

Now, to complete the proof of Theorem 3.6, we only need to prove (3.58). From (3.15) and (2.2), we see that

$$
\left(\sum_{j=1}^{q} 2\left|\lambda_{j}\right| d \bar{z}_{j}^{\wedge} d \bar{z}_{j}^{\wedge, *}+\sum_{j=q+1}^{n} 2\left|\lambda_{j}\right| d \bar{z}_{j}^{\wedge, *} d \bar{z}_{j}^{\wedge}\right) b_{1}(0,0)=\sum_{j=1}^{n} \frac{\partial^{2} b_{0}}{\partial z_{j} \partial \bar{z}_{j}}(0,0) .
$$

From (3.57), we see that

$$
\begin{aligned}
\sum_{j=1}^{n} \frac{\partial^{2} b_{0}}{\partial z_{j} \partial \bar{z}_{j}}(0,0)= & \sum_{q+1 \leq j \leq n, 1 \leq k \leq q, 1 \leq s \leq q} \frac{\left|\lambda_{s}\right|}{\left(\left|\lambda_{j}\right|+\left|\lambda_{k}\right|\right)\left(\left|\lambda_{j}\right|+\left|\lambda_{k}\right|+\left|\lambda_{s}\right|\right)^{2}} \\
& \times\left|\frac{\partial^{3} \phi}{\partial \bar{z}_{j} \partial z_{k} \partial z_{s}}(0)\right|^{2} d \bar{z}_{k}^{\wedge, *} d \bar{z}_{j}^{\wedge} b_{0}^{0} d \bar{z}_{j}^{\wedge, *} d \bar{z}_{k}^{\wedge} \\
& +\sum_{q+1 \leq j \leq n, 1 \leq k \leq q, q+1 \leq s \leq n} \frac{\left|\lambda_{s}\right|}{\left(\left|\lambda_{j}\right|+\left|\lambda_{k}\right|\right)\left(\left|\lambda_{j}\right|+\left|\lambda_{k}\right|+\left|\lambda_{s}\right|\right)^{2}} \\
& \times\left|\frac{\partial^{3} \phi}{\partial \bar{z}_{j} \partial z_{k} \partial \bar{z}_{s}}(0)\right|^{2} d \bar{z}_{k}^{\wedge, *} d \bar{z}_{j}^{\wedge} b_{0}^{0} d \bar{z}_{j}^{\wedge, *} d \bar{z}_{k}^{\wedge}+\alpha b_{0}^{0}+f,(3.61)
\end{aligned}
$$

where $\alpha \in \mathbb{C}$ and $\operatorname{Tr} f=0$. Since we can solve (3.60), we conclude that $\alpha=0$. Thus, (3.60) becomes:

$$
\begin{aligned}
& \left(\sum_{j=1}^{q} 2\left|\lambda_{j}\right| d \bar{z}_{j}^{\wedge} d \bar{z}_{j}^{\wedge, *}+\sum_{j=q+1}^{n} 2\left|\lambda_{j}\right| d \bar{z}_{j}^{\wedge, *} d \bar{z}_{j}^{\wedge}\right) b_{1}(0,0) \\
& =\sum_{q+1 \leq j \leq n, 1 \leq k \leq q, 1 \leq s \leq q} \frac{\left|\lambda_{s}\right|}{\left(\left|\lambda_{j}\right|+\left|\lambda_{k}\right|\right)\left(\left|\lambda_{j}\right|+\left|\lambda_{k}\right|+\left|\lambda_{s}\right|\right)^{2}}
\end{aligned}
$$




$$
\begin{array}{r}
\times\left|\frac{\partial^{3} \phi}{\partial \bar{z}_{j} \partial z_{k} \partial z_{s}}(0)\right|^{2} d \bar{z}_{k}^{\wedge, *} d \bar{z}_{j}^{\wedge} b_{0}^{0} d \bar{z}_{j}^{\wedge, *} d \bar{z}_{k}^{\wedge} \\
+\sum_{q+1 \leq j \leq n, 1 \leq k \leq q, q+1 \leq s \leq n} \frac{\left|\lambda_{s}\right|}{\left(\left|\lambda_{j}\right|+\left|\lambda_{k}\right|\right)\left(\left|\lambda_{j}\right|+\left|\lambda_{k}\right|+\left|\lambda_{s}\right|\right)^{2}} \\
\times\left|\frac{\partial^{3} \phi}{\partial \bar{z}_{j} \partial z_{k} \partial \bar{z}_{s}}(0)\right|^{2} d \bar{z}_{k}^{\wedge, *} d \bar{z}_{j}^{\wedge} b_{0}^{0} d \bar{z}_{j}^{\wedge, *} d \bar{z}_{k}^{\wedge}+f .
\end{array}
$$

Again, from Lemma 3.3, we get (3.58). Theorem 3.6 follows.

\section{The trace of the $b_{1}$ term}

As before, in this section, we assume that $\phi(z)=\sum_{j=1}^{n} \lambda_{j}\left|z_{j}\right|^{2}+O\left(|z|^{3}\right)$ near 0 and that $\lambda_{j}<0, j=1, \ldots, q$, and $\lambda_{j}>0, j=q+1, \ldots, n$. We work in some neighborhood of $(0,0)$ and we shall use the same notaions as before. In view of (3.58), we know that

$$
\begin{aligned}
b_{1}(0,0)= & \sum_{q+1 \leq j \leq n, 1 \leq k \leq q, 1 \leq s \leq q} \frac{\left|\lambda_{s}\right|}{2\left(\left|\lambda_{j}\right|+\left|\lambda_{k}\right|\right)^{2}\left(\left|\lambda_{j}\right|+\left|\lambda_{k}\right|+\left|\lambda_{s}\right|\right)^{2}} \\
& \times\left|\frac{\partial^{3} \phi}{\partial \bar{z}_{j} \partial z_{k} \partial z_{s}}(0)\right|^{2} d \bar{z}_{k}^{\wedge, *} d \bar{z}_{j}^{\wedge} b_{0}^{0} d \bar{z}_{j}^{\wedge, *} d \bar{z}_{k}^{\wedge} \\
& +\sum_{q+1 \leq j \leq n, 1 \leq k \leq q, q+1 \leq s \leq n} \frac{\left|\lambda_{s}\right|}{2\left(\left|\lambda_{j}\right|+\left|\lambda_{k}\right|\right)^{2}\left(\left|\lambda_{j}\right|+\left|\lambda_{k}\right|+\left|\lambda_{s}\right|\right)^{2}} \\
& \times\left|\frac{\partial^{3} \phi}{\partial \bar{z}_{j} \partial z_{k} \partial \bar{z}_{s}}(0)\right|^{2} d \bar{z}_{k}^{\wedge, *} d \bar{z}_{j}^{\wedge} b_{0}^{0} d \bar{z}_{j}^{\wedge, *} d \bar{z}_{k}^{\wedge}
\end{aligned}
$$

where $c \in \mathbb{C}, \operatorname{Tr} R=0$. The main goal of this section is to determine the constant $c$. We notice that the projection $\hat{\Pi}_{k}^{(q)}$ has the following property:

$$
\hat{\Pi}_{k}^{(q)} \circ \hat{\Pi}_{k}^{(q)}=\hat{\Pi}_{k}^{(q)}
$$

We recall that $\hat{\Pi}_{k}^{(q)}$ is given by (3.7). From (3.9), we have

$$
\hat{\Pi}_{k}^{(q)} \circ \hat{\Pi}_{k}^{(q)}(u, w)=\int e^{i k \psi(z, u)} b(u, z, k) e^{i k \psi(z, w)} b(z, w, k) d m(z)+F(u, w, k),
$$

where $F(u, w, k)$ is negligible. Take $u=w=0$ in (4.3) and from (4.2) and 
(3.9), we get

$$
\int e^{i k(\psi(0, z)+\psi(z, 0))} b(0, z, k) b(z, 0, k) d m(z) \sim k^{n} b_{0}(0,0)+k^{n-1} b_{1}(0,0)+\cdots
$$

We use $z_{j}=x_{2 j-1}+i x_{2 j}, j=1, \ldots, n$. We write $\partial_{x_{j}}$ to denote the operator $\frac{\partial}{\partial x_{j}}, j=1, \ldots, 2 n$. For multi-indix $\alpha=\left(\alpha_{1}, \ldots, \alpha_{2 n}\right), \alpha_{j} \in \mathbb{N} \bigcup\{0\}$, $j=1, \ldots, 2 n$. We write $|\alpha|=N$ if $\sum_{j=1}^{2 n} \alpha_{j}=N$ and we write $\partial_{x}^{\alpha}$ to denote the operator $\partial_{x_{1}}^{\alpha_{1}} \cdots \partial_{x_{2 n}}^{\alpha_{2 n}}$. We recall the stationary phase formula of Hörmander (see Theorem 7.7.5 in Hörmander [6])

Theorem 4.1. Let $K \subset \mathbb{C}^{n}$ be a compact set, $X$ an open neighborhood of $K$ and $N$ a positive integer. If $u \in C_{0}^{\infty}(K), f \in C^{\infty}(X)$ and $\operatorname{Im} f \geq 0$ in $X, \operatorname{Im} f\left(x_{0}\right)=0, f^{\prime}\left(x_{0}\right)=0$, det $f^{\prime \prime}\left(x_{0}\right) \neq 0, f^{\prime} \neq 0$ in $K \backslash\left\{x_{0}\right\}$ then

$$
\begin{aligned}
& \left|\int u(z) e^{i k f(z)} d m-2^{n} e^{i k f\left(x_{0}\right)} \operatorname{det}\left(\frac{k f^{\prime \prime}\left(x_{0}\right)}{2 \pi i}\right)^{-\frac{1}{2}} \sum_{j<N} k^{-j} L_{j} u\right| \\
& \quad \leq C k^{-N} \sum_{|\alpha| \leq 2 N} \sup \left|\partial_{x}^{\alpha} u\right|, \quad k>0,
\end{aligned}
$$

where $C$ is bounded when $f$ stays in a bounded set in $C^{\infty}(X)$ and $\frac{\left|x-x_{0}\right|}{\left|f^{\prime}(x)\right|}$ has a uniform bounded and

$$
L_{j} u=\sum_{\nu-\mu=j} \sum_{2 \nu \geq 3 \mu} i^{-j} 2^{-\nu}<f^{\prime \prime}\left(x_{0}\right)^{-1} D, D>^{\nu} \frac{\left(g_{x_{0}}^{\mu} u\right)\left(x_{0}\right)}{\nu ! \mu !} .
$$

Here

$$
\begin{aligned}
& \qquad g_{x_{0}}(x)=f(x)-f\left(x_{0}\right)-\frac{1}{2}<f^{\prime \prime}\left(x_{0}\right)\left(x-x_{0}\right), x-x_{0}> \\
& \text { and } D=\left(\begin{array}{c}
-i \partial_{x_{1}} \\
\vdots \\
-i \partial_{x_{2 n}}
\end{array}\right) \text {. }
\end{aligned}
$$

Now, we apply (4.5) to the left side of (4.4). From (2.2), we know that

$$
\psi(z, 0)+\psi(0, z)=2 i \operatorname{Im} \psi(z, 0)=2 i \sum_{j=1}^{n}\left|\lambda_{j}\right|\left|z_{j}\right|^{2}+O\left(|z|^{3}\right) .
$$


Since $\operatorname{Im}(\psi(0, z)+\psi(z, 0))>0$ when $z \neq 0$, we may assume that $b(0, z, k)$ has compact supports in some small neighborhood $K$ of $0 \in \mathbb{C}^{n}$. From (4.5), we have

$$
\begin{aligned}
& \int e^{i k(\psi(0, z)+\psi(z, 0))} b(0, z, k) b(z, 0, k) d m(z) \\
& =\left|\lambda_{1}\right|^{-1} \cdots\left|\lambda_{n}\right|^{-1} \pi^{n} k^{-n}\left(b(0,0, k)^{2}+\left.k^{-1} L_{1}(b(0, z, k) b(z, 0, k))\right|_{z=0}\right. \\
& \left.\quad+O\left(k^{2 n-2}\right)\right) .
\end{aligned}
$$

We can check that

$$
b(0,0, k)^{2}=k^{2 n} b_{0}(0,0)^{2}+k^{2 n-1}\left(b_{0}(0,0) b_{1}(0,0)+b_{1}(0,0) b_{0}(0,0)\right)+O\left(k^{2 n-2}\right) .
$$

The computation of the term $\left.L_{1}(b(0, z, k) b(z, 0, k))\right|_{z=0}$ is straight forward. We omit the process. We state our result

Proposition 4.2. Under the notations above, we have

$$
\begin{aligned}
k^{-2 n} & \left.L_{1}(b(0, z, k) b(z, 0, k))\right|_{z=0}+O\left(k^{-1}\right) \\
= & \frac{1}{2} \sum_{j=1}^{n} \frac{1}{\left|\lambda_{j}\right|}\left(\left.\frac{\partial^{2} b_{0}}{\partial z_{j} \partial \bar{z}_{j}}(z, 0)\right|_{z=0} b_{0}(0,0)+\left.\frac{\partial^{2} b_{0}}{\partial z_{j} \partial \bar{z}_{j}}(0, z)\right|_{z=0} b_{0}(0,0)\right. \\
& \left.+\left.\left.\left(\frac{\partial b_{0}}{\partial \bar{z}_{j}}\right)(0, z)\right|_{z=0} \frac{\partial b_{0}}{\partial z_{j}}(z, 0)\right|_{z=0}+\left.\left.\frac{\partial b_{0}}{\partial z_{j}}(0, z)\right|_{z=0} \frac{\partial b_{0}}{\partial \bar{z}_{j}}(z, 0)\right|_{z=0}\right) \\
& -\left.\frac{1}{4} \sum_{j, t=1}^{n} \frac{1}{\left|\lambda_{j}\right|\left|\lambda_{t}\right|} \frac{\partial^{4} \operatorname{Im} \psi(z, 0)}{\partial \bar{z}_{j} \partial z_{j} \partial \bar{z}_{t} \partial z_{t}}\right|_{z=0} b_{0}(0,0)^{2} \\
& -\left.\frac{1}{2} \sum_{j, s=1}^{n} \frac{1}{\left|\lambda_{j}\right|\left|\lambda_{s}\right|} \frac{\partial^{3} \operatorname{Im} \psi(z, 0)}{\partial \bar{z}_{j} \partial z_{j} \partial \bar{z}_{s}}\right|_{z=0}\left(\left.\frac{\partial b_{0}}{\partial z_{s}}(0, z)\right|_{z=0}+\left.\frac{\partial b_{0}}{\partial z_{s}}(z, 0)\right|_{z=0}\right) b_{0}(0,0) \\
& -\left.\frac{1}{2} \sum_{j, s=1}^{n} \frac{1}{\left|\lambda_{j}\right|\left|\lambda_{s}\right|} \frac{\partial^{3} \operatorname{Im} \psi(z, 0)}{\partial \bar{z}_{j} \partial z_{j} \partial z_{s}}\right|_{z=0}\left(\left.\frac{\partial b_{0}}{\partial \bar{z}_{s}}(0, z)\right|_{z=0}+\left.\frac{\partial b_{0}}{\partial \bar{z}_{s}}(z, 0)\right|_{z=0}\right) b_{0}(0,0) \\
& +\left.\frac{1}{4} \sum_{j, t, s=1}^{n} \frac{1}{\left|\lambda_{j}\right|\left|\lambda_{t}\right|\left|\lambda_{s}\right|}\left|\frac{\partial^{3} \operatorname{Im} \psi(z, 0)}{\partial z_{j} \partial z_{t} \partial \bar{z}_{s}}\right|_{z=0}\right|^{2} b_{0}(0,0)^{2} \\
& +\left.\left.\frac{1}{2} \sum_{j, t, s=1}^{n} \frac{1}{\left|\lambda_{j}\right|\left|\lambda_{t}\right|\left|\lambda_{s}\right|} \frac{\partial^{3} \operatorname{Im} \psi(z, 0)}{\partial \bar{z}_{j} \partial z_{j} \partial \bar{z}_{s}}\right|_{z=0} \frac{\partial^{3} \operatorname{Im} \psi(z, 0)}{\partial \bar{z}_{t} \partial z_{t} \partial z_{s}}\right|_{z=0} b_{0}(0,0)^{2}
\end{aligned}
$$




$$
+\left.\frac{1}{12} \sum_{j, k, s=1}^{n} \frac{1}{\left|\lambda_{j}\right|\left|\lambda_{t}\right|\left|\lambda_{s}\right|}\left|\frac{\partial^{3} \operatorname{Im} \psi(z, 0)}{\partial z_{j} \partial z_{t} \partial z_{s}}\right|_{z=0}\right|^{2} b_{0}(0,0)^{2}
$$

From (4.4), (4.9), (4.10) and (4.11), we get the following

Theorem 4.3. We have

$$
b_{1}(0,0)=\left|\lambda_{1}\right|^{-1} \cdots\left|\lambda_{n}\right|^{-1} \pi^{n}\left(b_{1}(0,0) b_{0}(0,0)+b_{0}(0,0) b_{1}(0,0)+C_{0}\right) \text {, }
$$

where $C_{0}$ denote the right side of (4.11).

For $A, B \in \mathscr{L}\left(\Lambda^{0, q} T_{0}^{*}\left(\mathbb{C}^{n}\right), \Lambda^{0, q} T_{0}^{*}\left(\mathbb{C}^{n}\right)\right)$, we write $A \perp B$ if $(A u \mid B u)=0$ for all $u \in \Lambda^{0, q} T_{0}^{*}\left(\mathbb{C}^{n}\right)$. For $F \in \mathscr{L}\left(\Lambda^{0, q} T_{0}^{*}\left(\mathbb{C}^{n}\right), \Lambda^{0, q} T_{0}^{*}\left(\mathbb{C}^{n}\right)\right)$, we werit $\hat{F}$ to denote the component of $A$ in the direction $d \bar{z}_{1}^{\wedge} d \bar{z}_{1}^{\wedge, *} \cdots d \bar{z}_{q}^{\wedge} d \bar{z}_{q}^{\wedge, *}$. More precisely, if

$$
F=\alpha d \bar{z}_{1}^{\wedge} d \bar{z}_{1}^{\wedge, *} \cdots d \bar{z}_{q}^{\wedge} d \bar{z}_{q}^{\wedge, *}+G, \quad \alpha \in \mathbb{C}, \quad G \perp d \bar{z}_{1}^{\wedge} d \bar{z}_{1}^{\wedge, *} \cdots d \bar{z}_{q}^{\wedge} d \bar{z}_{q}^{\wedge, *}
$$

then $\hat{F}=\alpha d \bar{z}_{1}^{\wedge} d \bar{z}_{1}^{\wedge, *} \cdots d \bar{z}_{q}^{\wedge} d \bar{z}_{q}^{\wedge, *}$. Now, we are ready to compute the constant $c$. We recall that $c$ is given by (4.1). From (4.1), we know that

$$
\hat{b}_{1}(0,0)=c b_{0}(0,0)
$$

Note that $b_{0}(0,0)=b_{0}^{0}$. From (4.1), (4.12) and (4.14), we get

$$
-c b_{0}(0,0)=\left|\lambda_{1}\right|^{-1} \cdots\left|\lambda_{n}\right|^{-1} \pi^{n} \hat{C}_{0} .
$$

From (4.15) and (4.11), we can determine $c$. The computation is very straightforward. We omit the detail and we refer the reader to Section 4 in [10] for the details. We state our result

Theorem 4.4. For $c$ in (4.1), we have

$$
\begin{aligned}
c= & \frac{1}{4}\left(-\sum_{1 \leq j, t \leq q} \frac{1}{\left|\lambda_{j}\right|\left|\lambda_{t}\right|} \frac{\partial^{4} \phi}{\partial \bar{z}_{j} \partial z_{j} \partial \bar{z}_{t} \partial z_{t}}(0)+\sum_{q+1 \leq j, t \leq n} \frac{1}{\left|\lambda_{j}\right|\left|\lambda_{t}\right|} \frac{\partial^{4} \phi}{\partial \bar{z}_{j} \partial z_{j} \partial \bar{z}_{t} \partial z_{t}}(0)\right. \\
& \left.-2 \sum_{q+1 \leq j \leq n, 1 \leq t \leq q} \frac{\left|\lambda_{j}\right|-\left|\lambda_{t}\right|}{\left|\lambda_{j}\right|\left|\lambda_{t}\right|\left(\left|\lambda_{j}\right|+\left|\lambda_{t}\right|\right)} \frac{\partial^{4} \phi}{\partial \bar{z}_{j} \partial z_{j} \partial \bar{z}_{t} \partial z_{t}}(0)\right)
\end{aligned}
$$




$$
\begin{aligned}
& -\frac{1}{2} \sum_{q+1 \leq j \leq n, 1 \leq t, s \leq q} \frac{\left|\lambda_{j}\right|-\left|\lambda_{t}\right|}{\left|\lambda_{j}\right|\left|\lambda_{t}\right|\left(\left|\lambda_{j}\right|+\left|\lambda_{t}\right|\right)\left(\left|\lambda_{s}\right|+\left|\lambda_{j}\right|\right)}\left|\frac{\partial^{3} \phi}{\partial \bar{z}_{s} \partial z_{j} \partial z_{t}}(0)\right|^{2} \\
& +\frac{1}{2} \sum_{q+1 \leq j, s \leq n, 1 \leq t \leq q} \frac{\left|\lambda_{j}\right|-\left|\lambda_{t}\right|}{\left|\lambda_{j}\right|\left|\lambda_{t}\right|\left(\left|\lambda_{j}\right|+\left|\lambda_{t}\right|\right)\left(\left|\lambda_{s}\right|+\left|\lambda_{t}\right|\right)}\left|\frac{\partial^{3} \phi}{\partial \bar{z}_{s} \partial z_{j} \partial z_{t}}(0)\right|^{2} \\
& +\frac{1}{4} \sum_{q+1 \leq j, t \leq n, 1 \leq s \leq q}\left(\frac{\left|\lambda_{t}\right|^{2}\left|\lambda_{j}\right|^{2}}{\left|\lambda_{s}\right|\left|\lambda_{j}\right|\left|\lambda_{t}\right|\left(\left|\lambda_{t}\right|+\left|\lambda_{j}\right|+\left|\lambda_{s}\right|\right)^{2}}\left(\frac{1}{\left|\lambda_{j}\right|+\left|\lambda_{s}\right|}+\frac{1}{\left|\lambda_{t}\right|+\left|\lambda_{s}\right|}\right)^{2}\right. \\
& \left.+\frac{1}{\left(\left|\lambda_{t}\right|+\left|\lambda_{j}\right|+\left|\lambda_{s}\right|\right)\left|\lambda_{j}\right|\left|\lambda_{t}\right|}\right)\left|\frac{\partial^{3} \phi}{\partial \bar{z}_{s} \partial z_{j} \partial z_{t}}(0)\right|^{2} \\
& +\frac{1}{4} \sum_{q+1 \leq s \leq n, 1 \leq j, t \leq q}\left(\frac{\left|\lambda_{t}\right|^{2}\left|\lambda_{j}\right|^{2}}{\left|\lambda_{s}\right|\left|\lambda_{j}\right|\left|\lambda_{t}\right|\left(\left|\lambda_{t}\right|+\left|\lambda_{j}\right|+\left|\lambda_{s}\right|\right)^{2}}\left(\frac{1}{\left|\lambda_{j}\right|+\left|\lambda_{s}\right|}+\frac{1}{\left|\lambda_{t}\right|+\left|\lambda_{s}\right|}\right)^{2}\right. \\
& \left.+\frac{1}{\left(\left|\lambda_{t}\right|+\left|\lambda_{j}\right|+\left|\lambda_{s}\right|\right)\left|\lambda_{j}\right|\left|\lambda_{t}\right|}\right)\left|\frac{\partial^{3} \phi}{\partial \bar{z}_{s} \partial z_{j} \partial z_{t}}(0)\right|^{2} \\
& -\frac{1}{4} \sum_{1 \leq j, t, s \leq q} \frac{1}{\left|\lambda_{j}\right|\left|\lambda_{t}\right|\left|\lambda_{s}\right|}\left|\frac{\partial^{3} \phi}{\partial \bar{z}_{s} \partial z_{j} \partial z_{t}}(0)\right|^{2} \\
& -\frac{1}{4} \sum_{q+1 \leq j, t, s \leq n} \frac{1}{\left|\lambda_{j}\right|\left|\lambda_{t}\right|\left|\lambda_{s}\right|}\left|\frac{\partial^{3} \phi}{\partial \bar{z}_{s} \partial z_{j} \partial z_{t}}(0)\right|^{2} \\
& +\frac{1}{2} \sum_{q+1 \leq j, t \leq n, 1 \leq s \leq q} \frac{\left|\lambda_{s}\right|}{\left|\lambda_{j}\right|\left|\lambda_{t}\right|\left(\left|\lambda_{j}\right|+\left|\lambda_{s}\right|\right)\left(\left|\lambda_{t}\right|+\left|\lambda_{s}\right|\right)} \frac{\partial^{3} \phi}{\partial \bar{z}_{j} \partial z_{j} \partial z_{s}}(0) \frac{\partial^{3} \phi}{\partial \bar{z}_{t} \partial z_{t} \partial \bar{z}_{s}}(0) \\
& +\frac{1}{2} \sum_{1 \leq j, t \leq q, q+1 \leq s \leq n} \frac{\left|\lambda_{s}\right|}{\left|\lambda_{j}\right|\left|\lambda_{t}\right|\left(\left|\lambda_{j}\right|+\left|\lambda_{s}\right|\right)\left(\left|\lambda_{t}\right|+\left|\lambda_{s}\right|\right)} \frac{\partial^{3} \phi}{\partial \bar{z}_{j} \partial z_{j} \partial z_{s}}(0) \frac{\partial^{3} \phi}{\partial \bar{z}_{t} \partial z_{t} \partial \bar{z}_{s}}(0) \\
& -\frac{1}{2} \sum_{1 \leq s, t \leq q, q+1 \leq j \leq n} \frac{1}{\left(\left|\lambda_{j}\right|+\left|\lambda_{t}\right|\right)\left(\left|\lambda_{j}\right|+\left|\lambda_{s}\right|\right)\left|\lambda_{t}\right|} \\
& \times\left(\frac{\partial^{3} \phi}{\partial \bar{z}_{j} \partial z_{j} \partial z_{s}}(0) \frac{\partial^{3} \phi}{\partial \bar{z}_{t} \partial z_{t} \partial \bar{z}_{s}}(0)+\frac{\partial^{3} \phi}{\partial \bar{z}_{j} \partial z_{j} \partial z_{s}}(0) \frac{\partial^{3} \phi}{\partial \bar{z}_{t} \partial z_{t} \partial \bar{z}_{s}}(0)\right) \\
& -\frac{1}{2} \sum_{1 \leq t \leq q, q+1 \leq j, s \leq n} \frac{1}{\left(\left|\lambda_{j}\right|+\left|\lambda_{t}\right|\right)\left(\left|\lambda_{t}\right|+\left|\lambda_{s}\right|\right)\left|\lambda_{j}\right|} \\
& \times\left(\frac{\partial^{3} \phi}{\partial \bar{z}_{j} \partial z_{j} \partial z_{s}}(0) \frac{\partial^{3} \phi}{\partial \bar{z}_{t} \partial z_{t} \partial \bar{z}_{s}}(0)+\frac{\partial^{3} \phi}{\partial \bar{z}_{j} \partial z_{j} \partial z_{s}}(0) \frac{\partial^{3} \phi}{\partial \bar{z}_{t} \partial z_{t} \partial \bar{z}_{s}}(0)\right) .
\end{aligned}
$$




\section{The end of the proof of Theorem 1.3}

In this section, we will use the same notations as in section 1 . Let

$$
F: \Lambda^{1,0} T\left(\mathbb{C}^{n}\right) \rightarrow \Lambda^{1,0} T\left(\mathbb{C}^{n}\right) \otimes \Lambda^{p_{1}, q_{1}} T^{*}\left(\mathbb{C}^{n}\right)
$$

and

$$
\left.T: \Lambda^{1,0} T\left(\mathbb{C}^{n}\right)\right) \rightarrow \Lambda^{1,0} T\left(\mathbb{C}^{n}\right) \otimes \Lambda^{p_{2}, q_{2}} T^{*}\left(\mathbb{C}^{n}\right)
$$

be linear operaors, where $p_{1}, q_{1}, p_{2}, q_{2} \in \mathbb{Z}, p_{1}, q_{1}, p_{2}, q_{2} \geq 0$. We write $F=$ $\left(F_{j, k}\right)_{j, k=1}^{n}, F_{j, k} \in \Lambda^{p_{1}, q_{1}} T^{*}\left(\mathbb{C}^{n}\right), 1 \leq j, k \leq n$, and $T=\left(T_{j, k}\right)_{j, k=1}^{n}, T_{j, k} \in$ $\Lambda^{p_{2}, q_{2}} T^{*}\left(\mathbb{C}^{n}\right), j, k=1, \ldots, n$, as in (1.17). $T F: \Lambda^{1,0} T\left(\mathbb{C}^{n}\right) \rightarrow \Lambda^{1,0} T\left(\mathbb{C}^{n}\right) \otimes$ $\Lambda^{p_{1}+p_{2}, q_{1}+q_{2}} T^{*}\left(\mathbb{C}^{n}\right)$ is the linear operator defined by $T F \frac{\partial}{\partial z_{k}}=\sum_{s, j=1}^{n} \frac{\partial}{\partial z_{j}} \otimes$ $\left(T_{j, s} \wedge F_{s, k}\right), k=1, \ldots, n$. We have $T F=\left(a_{j, k}\right)_{j, k=1}^{n}$. We can compute

$$
a_{j, k}=\sum_{s=1}^{n} T_{j, s} \wedge F_{s, k} \in \Lambda^{p_{1}+p_{2}, q_{1}+q_{2}} T^{*}\left(\mathbb{C}^{n}\right), \quad j, k=1, \ldots, n .
$$

For $p \in \mathbb{C}^{n}$, we may assume that $p=0$ and $\phi(z)=\sum_{j=1}^{n} \lambda_{j}\left|z_{j}\right|^{2}+O\left(|z|^{3}\right)$ near 0 and as before we suppose that $\lambda_{j}<0, j=1, \ldots, q$, and $\lambda_{j}>0$, $j=q+1, \ldots, n$. Let $M_{\phi}: C^{\infty}\left(\mathbb{C}^{n} ; \Lambda^{1,0} T\left(\mathbb{C}^{n}\right)\right) \rightarrow C^{\infty}\left(\mathbb{C}^{n} ; \Lambda^{1,0} T\left(\mathbb{C}^{n}\right)\right)$ be as in (1.20). We recall that $M_{\phi}=\left(\frac{\partial^{2} \phi}{\partial \bar{z}_{j} \partial z_{k}}\right)_{j, k=1}^{n}$. We have

$$
U_{j}=\frac{\partial}{\partial z_{j}} \text { at } 0, \quad j=1, \ldots, n,
$$

where $U_{j}, j=1, \ldots, n$, are given by (1.22). Moreover, we have

$$
\begin{aligned}
& \left(\frac{\partial}{\partial z_{j}} \mid \frac{\partial}{\partial z_{k}}\right)_{|\phi|}=\left(\frac{\partial}{\partial \bar{z}_{j}} \mid \frac{\partial}{\partial \bar{z}_{k}}\right)_{|\phi|}=\delta_{j, k}\left|\lambda_{j}\right| \text { at } 0, \quad j, k=1, \ldots, n, \\
& \left(d z_{j} \mid d z_{k}\right)_{|\phi|}=\left(d \bar{z}_{j} \mid d \bar{z}_{k}\right)_{|\phi|}=\delta_{j, k} \frac{1}{\left|\lambda_{j}\right|} \text { at } 0, \quad j, k=1, \ldots, n .
\end{aligned}
$$

Here $\delta_{j, k}=1$ if $j=k, \delta_{j, k}=0$ if $j \neq k$. For the definition of the Hermitian metrix $(\mid)_{|\phi|}$, see the discussion after (1.22). 
Put $M_{\phi}^{-1}(z)=\left(b_{j, k}(z)\right)_{j, k=1}^{n}$. We claim that

$$
b_{j, k}(z)=\frac{\delta_{j, k}}{\lambda_{j}}-\frac{1}{\lambda_{j} \lambda_{k}} \sum_{s=1}^{n}\left(\frac{\partial^{3} \phi}{\partial \bar{z}_{j} \partial z_{k} \partial z_{s}}(0) z_{s}+\frac{\partial^{3} \phi}{\partial \bar{z}_{j} \partial z_{k} \partial \bar{z}_{s}}(0) \bar{z}_{s}\right)+O\left(|z|^{2}\right),
$$

$j, k=1, \ldots, n$, near $0 \in \mathbb{C}^{n}$. In fact, if we put $\widetilde{B}=\left(\widetilde{b}_{j, k}\right)_{j, k=1}^{n}, \widetilde{b}_{j, k}=$ $\frac{\delta_{j, k}}{\lambda_{j}}-\frac{1}{\lambda_{j} \lambda_{k}} \sum_{s=1}^{n}\left(\frac{\partial^{3} \phi}{\partial \bar{z}_{j} \partial z_{k} \partial z_{s}}(0) z_{s}+\frac{\partial^{3} \phi}{\partial \bar{z}_{j} \partial z_{k} \partial \bar{z}_{s}}(0) \bar{z}_{s}\right)$. Then $M_{\phi} \widetilde{B}=C, C=$ $\left(c_{j, k}\right)_{j, k=1}^{n}$,

$$
\begin{aligned}
c_{j, k} & =\sum_{t=1}^{n} \frac{\partial^{2} \phi}{\partial \bar{z}_{j} \partial z_{t}}(z) \widetilde{b}_{t, k}(z) \\
& =\sum_{t=1}^{n} \frac{\partial^{2} \phi}{\partial \bar{z}_{j} \partial z_{t}}(z)\left(\frac{\delta_{t, k}}{\lambda_{t}}-\frac{1}{\lambda_{t} \lambda_{k}} \sum_{s=1}^{n}\left(\frac{\partial^{3} \phi}{\partial \bar{z}_{t} \partial z_{k} \partial z_{s}}(0) z_{s}+\frac{\partial^{3} \phi}{\partial \bar{z}_{t} \partial z_{k} \partial \bar{z}_{s}}(0) \bar{z}_{s}\right)\right) \\
& =\sum_{t=1}^{n}\left(\lambda_{t} \delta_{j, t}+\sum_{s=1}^{n}\left(\frac{\partial^{3} \phi}{\partial \bar{z}_{j} \partial z_{t} \partial z_{s}}(0) z_{s}+\frac{\partial^{3} \phi}{\partial \bar{z}_{j} \partial z_{t} \partial \bar{z}_{s}}(0) \bar{z}_{s}\right)\right) \\
& \times\left(\frac{\delta_{t, k}}{\lambda_{t}}-\frac{1}{\lambda_{t} \lambda_{k}} \sum_{s=1}^{n}\left(\frac{\partial^{3} \phi}{\partial \bar{z}_{t} \partial z_{k} \partial z_{s}}(0) z_{s}+\frac{\partial^{3} \phi}{\partial \bar{z}_{t} \partial z_{k} \partial \bar{z}_{s}}(0) \bar{z}_{s}\right)\right)+O\left(|z|^{2}\right) \\
& =\delta_{j, k}+O\left(|z|^{2}\right) .
\end{aligned}
$$

Thus, $\widetilde{B}=M_{\phi}^{-1}(z)+O\left(|z|^{2}\right)$. (15.5) follows.

Let $Q: \Lambda^{1,0} T\left(\mathbb{C}^{n}\right) \rightarrow \Lambda^{1,0} T^{*}\left(\mathbb{C}^{n}\right) \otimes \Lambda^{1,0} T\left(\mathbb{C}^{n}\right)$ be as in (1.26). We write $Q=\left(Q_{j, k}\right)_{j, k=1}^{n}, Q_{j, k}(z)=\sum_{s=1}^{n} \frac{\partial^{3} \phi}{\partial \bar{z}_{j} \partial z_{k} \partial z_{s}}(0) q_{j, k, s} d z_{s}$ at 0, where

$$
\begin{aligned}
q_{j, k, s}= & \frac{\left|\lambda_{k}\right| \delta_{j}(k)+\left|\lambda_{s}\right| \delta_{j}(s)}{\left|\lambda_{j}\right|+\left|\lambda_{k}\right| \delta_{j}(k)+\left|\lambda_{s}\right| \delta_{j}(s)}-\delta_{j}(k) \delta_{j}(s) \\
& \times \frac{\left|\lambda_{k}\right|^{2}\left|\lambda_{s}\right|^{2}}{\left(\left|\lambda_{j}\right|+\left|\lambda_{k}\right|+\left|\lambda_{s}\right|\right)^{2}}\left(\frac{1}{\left|\lambda_{j}\right|+\left|\lambda_{k}\right|}+\frac{1}{\left|\lambda_{j}\right|+\left|\lambda_{s}\right|}\right)^{2} .
\end{aligned}
$$

We recall that the definition of $\delta_{k}(j)$ is given by (1.25). Put $\bar{\partial} M_{\phi}^{-1}=$ $\left(d_{j, k}\right)_{j, k=1}^{n}$. From (5.5), we see that

$$
d_{j, k}=-\sum_{s=1}^{n} \frac{1}{\lambda_{j} \lambda_{k}} \frac{\partial^{3} \phi}{\partial \bar{z}_{j} \partial z_{k} \partial \bar{z}_{s}}(0) d \bar{z}_{s}
$$


at 0 . Put $\bar{\partial} M_{\phi}^{-1} Q=\left(f_{j, k}\right)_{j, k=1}^{n}$. From (5.1), (5.8), we see that

$$
f_{k, j}=-\sum_{1 \leq t, s, u \leq n} \frac{1}{\lambda_{k} \lambda_{t}} q_{t, j, u} \frac{\partial^{3} \phi}{\partial \bar{z}_{k} \partial z_{t} \partial \bar{z}_{s}}(0) \frac{\partial^{3} \phi}{\partial \bar{z}_{t} \partial z_{j} \partial z_{u}}(0) d \bar{z}_{s} \wedge d z_{u}
$$

at 0 . As in section 1 , put $e_{j}=\frac{1}{\sqrt{\left|\lambda_{j}\right|}} U_{j}, j=1, \ldots, n$. From (5.9), it is not difficult to see that

$$
<\left(\bar{\partial} M_{\phi}^{-1} Q e_{j} \mid e_{k}\right)_{|\phi|}, \bar{e}_{j} \wedge e_{k}>(0)=-\sum_{1 \leq t \leq n} \frac{1}{\lambda_{k} \lambda_{t}\left|\lambda_{j}\right|} q_{t, j, k}\left|\frac{\partial^{3} \phi}{\partial \bar{z}_{k} \partial z_{t} \partial \bar{z}_{j}}(0)\right|^{2}
$$

$j, k=1, \ldots, n$. From (5.10) and (5.7), it is straight forward to see that

Proposition 5.1. We have that

$$
\begin{aligned}
& <\left(\bar{\partial} M_{\phi}^{-1} Q e_{j} \mid e_{k}\right)_{|\phi|}, \bar{e}_{j} \wedge e_{k}>(0) \\
& =\sum_{1 \leq t \leq q} \frac{1}{\left|\lambda_{t}\right|\left|\lambda_{j}\right|\left|\lambda_{k}\right|}\left|\frac{\partial^{3} \phi}{\partial \bar{z}_{k} \partial z_{t} \partial \bar{z}_{j}}(0)\right|^{2} \\
& \times\left(\frac{\left|\lambda_{j}\right|+\left|\lambda_{k}\right|}{\left|\lambda_{j}\right|+\left|\lambda_{k}\right|+\left|\lambda_{t}\right|}-\frac{\left|\lambda_{j}\right|^{2}\left|\lambda_{k}\right|^{2}}{\left(\left|\lambda_{j}\right|+\left|\lambda_{k}\right|+\left|\lambda_{t}\right|\right)^{2}}\left(\frac{1}{\left|\lambda_{j}\right|+\left|\lambda_{t}\right|}+\frac{1}{\left|\lambda_{k}\right|+\left|\lambda_{t}\right|}\right)^{2}\right),
\end{aligned}
$$

where $q+1 \leq j, k \leq n$,

$$
\begin{aligned}
& <\left(\bar{\partial} M_{\phi}^{-1} Q e_{j} \mid e_{k}\right)_{|\phi|}, \bar{e}_{j} \wedge e_{k}>(0) \\
& =\sum_{q+1 \leq t \leq n} \frac{1}{\left|\lambda_{t}\right|\left|\lambda_{j}\right|\left|\lambda_{k}\right|}\left|\frac{\partial^{3} \phi}{\partial \bar{z}_{k} \partial z_{t} \partial \bar{z}_{j}}(0)\right|^{2} \\
& \times\left(\frac{\left|\lambda_{j}\right|+\left|\lambda_{k}\right|}{\left|\lambda_{j}\right|+\left|\lambda_{k}\right|+\left|\lambda_{t}\right|}-\frac{\left|\lambda_{j}\right|^{2}\left|\lambda_{k}\right|^{2}}{\left(\left|\lambda_{j}\right|+\left|\lambda_{k}\right|+\left|\lambda_{t}\right|\right)^{2}}\left(\frac{1}{\left|\lambda_{j}\right|+\left|\lambda_{t}\right|}+\frac{1}{\left|\lambda_{k}\right|+\left|\lambda_{t}\right|}\right)^{2}\right),
\end{aligned}
$$

where $1 \leq j, k \leq q$,

$$
\begin{aligned}
< & \left(\bar{\partial} M_{\phi}^{-1} Q e_{j} \mid e_{k}\right)_{|\phi|}, \bar{e}_{j} \wedge e_{k}>(0) \\
= & -\sum_{1 \leq t \leq q} \frac{1}{\left|\lambda_{t}\right|\left|\lambda_{j}\right|\left|\lambda_{k}\right|} \frac{\left|\lambda_{j}\right|}{\left|\lambda_{t}\right|+\left|\lambda_{j}\right|}\left|\frac{\partial^{3} \phi}{\partial \bar{z}_{k} \partial z_{t} \partial \bar{z}_{j}}(0)\right|^{2} \\
& +\sum_{q+1 \leq t \leq n} \frac{1}{\left|\lambda_{t}\right|\left|\lambda_{j}\right|\left|\lambda_{k}\right|} \frac{\left|\lambda_{k}\right|}{\left|\lambda_{t}\right|+\left|\lambda_{k}\right|}\left|\frac{\partial^{3} \phi}{\partial \bar{z}_{k} \partial z_{t} \partial \bar{z}_{j}}(0)\right|^{2},
\end{aligned}
$$


where $q+1 \leq j \leq n, 1 \leq k \leq q$, and

$$
\begin{aligned}
< & \left(\bar{\partial} M_{\phi}^{-1} Q e_{j} \mid e_{k}\right)_{|\phi|}, \bar{e}_{j} \wedge e_{k}>(0) \\
= & \sum_{1 \leq t \leq q} \frac{1}{\left|\lambda_{t}\right|\left|\lambda_{j}\right|\left|\lambda_{k}\right|} \frac{\left|\lambda_{k}\right|}{\left|\lambda_{t}\right|+\left|\lambda_{k}\right|}\left|\frac{\partial^{3} \phi}{\partial \bar{z}_{k} \partial z_{t} \partial \bar{z}_{j}}(0)\right|^{2} \\
& -\sum_{q+1 \leq t \leq n} \frac{1}{\left|\lambda_{t}\right|\left|\lambda_{j}\right|\left|\lambda_{k}\right|} \frac{\left|\lambda_{j}\right|}{\left|\lambda_{t}\right|+\left|\lambda_{j}\right|}\left|\frac{\partial^{3} \phi}{\partial \bar{z}_{k} \partial z_{t} \partial \bar{z}_{j}}(0)\right|^{2},
\end{aligned}
$$

where $1 \leq j \leq q, q+1 \leq k \leq n$.

Let $\Theta_{\phi}: C^{\infty}\left(\mathbb{C}^{n} ; \Lambda^{1,0} T\left(\mathbb{C}^{n}\right)\right) \rightarrow C^{\infty}\left(\mathbb{C}^{n} ; \Lambda^{1,1} T^{*}\left(\mathbb{C}^{n}\right) \otimes \Lambda^{1,0} T\left(\mathbb{C}^{n}\right)\right)$ be as in (1.24). We recall that $\Theta_{\phi}=\left(\bar{\partial} \theta_{j, k}\right)_{j, k=1}^{n}=\left(\Theta_{j, k}\right)_{j, k=1}^{n}$, where $\theta=$ $h^{-1} \partial h=\left(\theta_{j, k}\right)_{j, k=1}^{n}, h=\left(\frac{\partial^{2} \phi}{\partial \bar{z}_{j} \partial z_{k}}\right)_{j, k=1}^{n}$. It is not difficult to see that

$$
\Theta_{j, k}=\frac{1}{\lambda_{j}} \bar{\partial} \partial\left(\frac{\partial^{2} \phi}{\partial \bar{z}_{j} \partial z_{k}}\right)-\sum_{t=1}^{n} \frac{1}{\lambda_{t} \lambda_{j}} \bar{\partial}\left(\frac{\partial^{2} \phi}{\partial \bar{z}_{j} \partial z_{t}}\right) \wedge \partial\left(\frac{\partial^{2} \phi}{\partial \bar{z}_{t} \partial z_{k}}\right),
$$

$j, k=1, \ldots, n$. From (5.15), it is straight forward to see that

Proposition 5.2. We have that

$$
\begin{aligned}
< & \left(\Theta_{\phi} e_{j} \mid e_{k}\right)_{|\phi|}, \bar{e}_{j} \wedge e_{k}>(0) \\
= & \frac{1}{\left|\lambda_{j}\right|\left|\lambda_{k}\right|} \frac{\partial^{4} \phi}{\partial \bar{z}_{j} \partial z_{j} \partial \bar{z}_{k} \partial z_{k}}(0)+\sum_{t=1}^{q} \frac{1}{\left|\lambda_{t}\right|\left|\lambda_{j}\right|\left|\lambda_{k}\right|}\left|\frac{\partial^{3} \phi}{\partial \bar{z}_{j} \partial z_{t} \partial \bar{z}_{k}}(0)\right|^{2} \\
& -\sum_{t=q+1}^{n} \frac{1}{\left|\lambda_{t}\right|\left|\lambda_{j}\right|\left|\lambda_{k}\right|}\left|\frac{\partial^{3} \phi}{\partial \bar{z}_{j} \partial z_{t} \partial \bar{z}_{k}}(0)\right|^{2}
\end{aligned}
$$

where $q+1 \leq j, k \leq n$,

$$
\begin{aligned}
< & \left(\Theta_{\phi} e_{j} \mid e_{k}\right)_{|\phi|}, \bar{e}_{j} \wedge e_{k}>(0) \\
= & -\frac{1}{\left|\lambda_{j}\right|\left|\lambda_{k}\right|} \frac{\partial^{4} \phi}{\partial \bar{z}_{j} \partial z_{j} \partial \bar{z}_{k} \partial z_{k}}(0)-\sum_{t=1}^{q} \frac{1}{\left|\lambda_{t}\right|\left|\lambda_{j}\right|\left|\lambda_{k}\right|}\left|\frac{\partial^{3} \phi}{\partial \bar{z}_{j} \partial z_{t} \partial \bar{z}_{k}}(0)\right|^{2} \\
& +\sum_{t=q+1}^{n} \frac{1}{\left|\lambda_{t}\right|\left|\lambda_{j}\right|\left|\lambda_{k}\right|}\left|\frac{\partial^{3} \phi}{\partial \bar{z}_{j} \partial z_{t} \partial \bar{z}_{k}}(0)\right|^{2}
\end{aligned}
$$


where $1 \leq j, k \leq q$,

$$
\begin{aligned}
< & \left(\Theta_{\phi} e_{j} \mid e_{k}\right)_{|\phi|}, \bar{e}_{j} \wedge e_{k}>(0) \\
= & -\frac{1}{\left|\lambda_{j}\right|\left|\lambda_{k}\right|} \frac{\partial^{4} \phi}{\partial \bar{z}_{j} \partial z_{j} \partial \bar{z}_{k} \partial z_{k}}(0)-\sum_{t=1}^{q} \frac{1}{\left|\lambda_{t}\right|\left|\lambda_{j}\right|\left|\lambda_{k}\right|}\left|\frac{\partial^{3} \phi}{\partial \bar{z}_{j} \partial z_{t} \partial \bar{z}_{k}}(0)\right|^{2} \\
& +\sum_{t=q+1}^{n} \frac{1}{\left|\lambda_{t}\right|\left|\lambda_{j}\right|\left|\lambda_{k}\right|}\left|\frac{\partial^{3} \phi}{\partial \bar{z}_{j} \partial z_{t} \partial \bar{z}_{k}}(0)\right|^{2}
\end{aligned}
$$

where $q+1 \leq j \leq n, 1 \leq k \leq q$, and

$$
\begin{aligned}
< & \left(\Theta_{\phi} e_{j} \mid e_{k}\right)_{|\phi|}, \bar{e}_{j} \wedge e_{k}>(0) \\
= & \frac{1}{\left|\lambda_{j}\right|\left|\lambda_{k}\right|} \frac{\partial^{4} \phi}{\partial \bar{z}_{j} \partial z_{j} \partial \bar{z}_{k} \partial z_{k}}(0)+\sum_{t=1}^{q} \frac{1}{\left|\lambda_{t}\right|\left|\lambda_{j}\right|\left|\lambda_{k}\right|}\left|\frac{\partial^{3} \phi}{\partial \bar{z}_{j} \partial z_{t} \partial \bar{z}_{k}}(0)\right|^{2} \\
& -\sum_{t=q+1}^{n} \frac{1}{\left|\lambda_{t}\right|\left|\lambda_{j}\right|\left|\lambda_{k}\right|}\left|\frac{\partial^{3} \phi}{\partial \bar{z}_{j} \partial z_{t} \partial \bar{z}_{k}}(0)\right|^{2},
\end{aligned}
$$

where $1 \leq j \leq q, q+1 \leq k \leq n$.

As in section 1 , define

$$
R=\Theta_{\phi}-\left(\bar{\partial} M_{\phi}^{-1}\right) Q: \Lambda^{1,0} T\left(\mathbb{C}^{n}\right) \rightarrow \Lambda^{1,1} T^{*}\left(\mathbb{C}^{n}\right) \otimes \Lambda^{1,0} T\left(\mathbb{C}^{n}\right)
$$

From (5.11)-(5.14) and (5.16)-(5.17), it is not difficult to see that

$$
\begin{aligned}
& \frac{1}{4} \sum_{j, k=1}^{n}\left(1+\delta_{j}(k) \frac{\left|\lambda_{j}\right|-\left|\lambda_{k}\right|}{\left|\lambda_{j}\right|+\left|\lambda_{k}\right|}<\left(R e_{j} \mid e_{k}\right)_{|\phi|}, \bar{e}_{j} \wedge e_{k}>(0)\right. \\
& =\frac{1}{4}\left(-\sum_{1 \leq j, k \leq q} \frac{1}{\left|\lambda_{j}\right|\left|\lambda_{t}\right|} \frac{\partial^{4} \phi}{\partial \bar{z}_{j} \partial z_{j} \partial \bar{z}_{k} \partial z_{k}}(0)+\sum_{q+1 \leq j, k \leq n} \frac{1}{\left|\lambda_{j}\right|\left|\lambda_{k}\right|} \frac{\partial^{4} \phi}{\partial \bar{z}_{j} \partial z_{j} \partial \bar{z}_{k} \partial z_{k}}(0)\right. \\
& \left.-2 \sum_{q+1 \leq j \leq n, 1 \leq k \leq q} \frac{\left|\lambda_{j}\right|-\left|\lambda_{k}\right|}{\left|\lambda_{j}\right|\left|\lambda_{k}\right|\left(\left|\lambda_{j}\right|+\left|\lambda_{k}\right|\right)} \frac{\partial^{4} \phi}{\partial \bar{z}_{j} \partial z_{j} \partial \bar{z}_{k} \partial z_{k}}(0)\right) \\
& \quad-\frac{1}{2} \sum_{q+1 \leq j \leq n, 1 \leq k, s \leq q} \frac{\left|\lambda_{j}\right|-\left|\lambda_{k}\right|}{\left|\lambda_{j}\right|\left|\lambda_{k}\right|\left(\left|\lambda_{j}\right|+\left|\lambda_{k}\right|\right)\left(\left|\lambda_{s}\right|+\left|\lambda_{j}\right|\right)}\left|\frac{\partial^{3} \phi}{\partial \bar{z}_{s} \partial z_{j} \partial z_{k}}(0)\right|^{2} \\
& \quad+\frac{1}{2} \sum_{q+1 \leq j, s \leq n, 1 \leq k \leq q} \frac{\left|\lambda_{j}\right|-\left|\lambda_{k}\right|}{\left|\lambda_{j}\right|\left|\lambda_{k}\right|\left(\left|\lambda_{j}\right|+\left|\lambda_{k}\right|\right)\left(\left|\lambda_{s}\right|+\left|\lambda_{k}\right|\right)}\left|\frac{\partial^{3} \phi}{\partial \bar{z}_{s} \partial z_{j} \partial z_{k}}(0)\right|^{2}
\end{aligned}
$$




$$
\begin{aligned}
& +\frac{1}{4} \sum_{q+1 \leq j, k \leq n, 1 \leq s \leq q}\left(\frac { | \lambda _ { k } | ^ { 2 } | \lambda _ { j } | ^ { 2 } } { | \lambda _ { s } | | \lambda _ { j } | | \lambda _ { k } | ( | \lambda _ { k } | + | \lambda _ { j } | + | \lambda _ { s } | ) ^ { 2 } } \left(\frac{1}{\left|\lambda_{j}\right|+\left|\lambda_{s}\right|}\right.\right. \\
& \left.\left.+\frac{1}{\left|\lambda_{k}\right|+\left|\lambda_{s}\right|}\right)^{2}+\frac{1}{\left(\left|\lambda_{k}\right|+\left|\lambda_{j}\right|+\left|\lambda_{s}\right|\right)\left|\lambda_{j}\right|\left|\lambda_{k}\right|}\right)\left|\frac{\partial^{3} \phi}{\partial \bar{z}_{s} \partial z_{j} \partial z_{k}}(0)\right|^{2} \\
& +\frac{1}{4} \sum_{q+1 \leq s \leq n, 1 \leq j, k \leq q}\left(\frac{\left|\lambda_{k}\right|^{2}\left|\lambda_{j}\right|^{2}}{\left|\lambda_{s}\right|\left|\lambda_{j}\right|\left|\lambda_{k}\right|\left(\left|\lambda_{k}\right|+\left|\lambda_{j}\right|+\left|\lambda_{s}\right|\right)^{2}}\left(\frac{1}{\left|\lambda_{j}\right|+\left|\lambda_{s}\right|}+\frac{1}{\left|\lambda_{k}\right|+\left|\lambda_{s}\right|}\right)^{2}\right. \\
& \left.+\frac{1}{\left(\left|\lambda_{k}\right|+\left|\lambda_{j}\right|+\left|\lambda_{s}\right|\right)\left|\lambda_{j}\right|\left|\lambda_{k}\right|}\right)\left|\frac{\partial^{3} \phi}{\partial \bar{z}_{s} \partial z_{j} \partial z_{k}}(0)\right|^{2} \\
& -\frac{1}{4} \sum_{1 \leq j, k, s \leq q} \frac{1}{\left|\lambda_{j}\right|\left|\lambda_{k}\right|\left|\lambda_{s}\right|}\left|\frac{\partial^{3} \phi}{\partial \bar{z}_{s} \partial z_{j} \partial z_{k}}(0)\right|^{2} \\
& -\frac{1}{4} \sum_{q+1 \leq j, k, s \leq n} \frac{1}{\left|\lambda_{j}\right|\left|\lambda_{k}\right|\left|\lambda_{s}\right|}\left|\frac{\partial^{3} \phi}{\partial \bar{z}_{s} \partial z_{j} \partial z_{k}}(0)\right|^{2} .
\end{aligned}
$$

From (5.7), we can check that

$$
\left(Q e_{j} \mid e_{j}\right)=\sum_{s=1}^{n} \delta_{j}(s) \frac{\left|\lambda_{s}\right|}{\left|\lambda_{j}\right|\left(\left|\lambda_{j}\right|+\left|\lambda_{s}\right|\right)} \frac{\partial^{3} \phi}{\partial \bar{z}_{j} \partial z_{j} \partial z_{s}}(0) d z_{s}
$$

at 0 . From this, it is straight forward to see that

$$
\begin{aligned}
- & \sum_{j, k=1}^{n} \delta_{j}(k) \frac{\left|\lambda_{j}\right|}{\left|\lambda_{j}\right|+\left|\lambda_{k}\right|} \operatorname{Re}\left(\left(Q e_{j} \mid e_{j}\right)\left|\partial M_{\phi} e_{k}\right| e_{k}\right)_{|\phi|}(0) \\
= & -\frac{1}{2} \sum_{1 \leq s, k \leq q, q+1 \leq j \leq n} \frac{1}{\left(\left|\lambda_{j}\right|+\left|\lambda_{k}\right|\right)\left(\left|\lambda_{j}\right|+\left|\lambda_{s}\right|\right)\left|\lambda_{k}\right|} \\
& \times\left(\frac{\partial^{3} \phi}{\partial \bar{z}_{j} \partial z_{j} \partial z_{s}}(0) \frac{\partial^{3} \phi}{\partial \bar{z}_{k} \partial z_{k} \partial \bar{z}_{s}}(0)+\frac{\partial^{3} \phi}{\partial \bar{z}_{j} \partial z_{j} \partial z_{s}}(0) \frac{\partial^{3} \phi}{\partial \bar{z}_{k} \partial z_{k} \partial \bar{z}_{s}}(0)\right) \\
& -\frac{1}{2} \sum_{1 \leq k \leq q, q+1 \leq j, s \leq n} \frac{1}{\left(\left|\lambda_{j}\right|+\left|\lambda_{k}\right|\right)\left(\left|\lambda_{k}\right|+\left|\lambda_{s}\right|\right)\left|\lambda_{j}\right|} \\
& \times\left(\frac{\partial^{3} \phi}{\partial \bar{z}_{j} \partial z_{j} \partial z_{s}}(0) \frac{\partial^{3} \phi}{\partial \bar{z}_{k} \partial z_{k} \partial \bar{z}_{s}}(0)+\frac{\partial^{3} \phi}{\partial \bar{z}_{j} \partial z_{j} \partial z_{s}}(0) \frac{\partial^{3} \phi}{\partial \bar{z}_{k} \partial z_{k} \partial \bar{z}_{s}}(0)\right)
\end{aligned}
$$

and

$$
\frac{1}{2}\left|\sum_{j=1}^{n}\left(Q e_{j} \mid e_{j}\right)^{2}\right|
$$




$$
\begin{aligned}
=\frac{1}{2} \sum_{q+1 \leq j, k \leq n, 1 \leq s \leq q} & \frac{\left|\lambda_{s}\right|}{\left|\lambda_{j}\right|\left|\lambda_{k}\right|\left(\left|\lambda_{j}\right|+\left|\lambda_{s}\right|\right)\left(\left|\lambda_{k}\right|+\left|\lambda_{s}\right|\right)} \frac{\partial^{3} \phi}{\partial \bar{z}_{j} \partial z_{j} \partial z_{s}}(0) \\
& \times \frac{\partial^{3} \phi}{\partial \bar{z}_{k} \partial z_{k} \partial \bar{z}_{s}}(0) \\
+\frac{1}{2} \sum_{1 \leq j, k \leq q, q+1 \leq s \leq n} & \frac{\left|\lambda_{s}\right|}{\left|\lambda_{j}\right|\left|\lambda_{k}\right|\left(\left|\lambda_{j}\right|+\left|\lambda_{s}\right|\right)\left(\left|\lambda_{k}\right|+\left|\lambda_{s}\right|\right)} \frac{\partial^{3} \phi}{\partial \bar{z}_{j} \partial z_{j} \partial z_{s}}(0) \\
& \times \frac{\partial^{3} \phi}{\partial \bar{z}_{k} \partial z_{k} \partial \bar{z}_{s}}(0) .
\end{aligned}
$$

Combining (5.22), (5.21) and (5.20) with (4.16), Theorem 1.3 follows.

\section{Acknowledgments}

A large part of this paper has been carried out when the author was a postdoc fellow at Chalmers University of Technology during December 2008December 2010 supported by the Swedish research council. I am grateful to Bo Berndtsson and Robert Berman for several useful conversations and to the Department of Mathematics, Chalmers University of Technology and the University of Göteborg for offering excellent working conditions. I would like to thank Jih-Hsin Cheng and Chun-Chung Hsieh at the Institute of Mathematics of Academia Sinica, Taiwan, for several interesting discussions on the material of this paper. Furthermore, the author is grateful to George Marinescu for comments and useful suggestions on an early draft of the manuscript.

\section{References}

1. Berman, R. and Sjöstrand, J., Asymptotics for Bergman-Hodge kernels for high powers of complex line bundles, Ann. de la Fac. des Sciences de Toulouse, XIV(2007), 719771 .

2. Catlin, D., The Bergman kernel and a theorem of Tian, Analysis and geometry in several complex variables, (Katata, 1997). Trends in Math., Birkhauser, Boston, MA, 1999, 1-23.

3. Donaldson, S. K., Scalar curvature and projective embeddings. I, J. Differential Geom., 59 (2001), 479-522.

4. Fine, J., Calabi flow and projective embeddings, J. Differential Geom., 84 (2010), 489-523. 
5. Fine, J., Quantisation and the Hessian of Mabuchi Energy, available at 1009.4543.

6. Hörmander, L., The Analysis of Linear Partial Differential Operators I Distribution Theory And Fourier Analysis, Classics in Mathematics, Springer-Verlag, Berlin, 2003.

7. Hsiao, C-Y., Projections in several complex variables, Mém. Soc. Math. France, Nouv. Sér., 123 (2010), 131.

8. Hsiao, C-Y., On the coefficients of the asymptotic expansion of the kernel of BerezinToeplitz quantization, Annals of Global Analysis and Geometry, 42 (2012), 207-245.

9. Hsiao, C-Y. and Marinescu, G., Asymptotics of spectral function of lower energy forms and Bergman kernel of semi-positive and big line bundles, Commun. Anal. Geom., 22 (2014), 1-108.

10. Hsiao, C-Y., The second coefficient of the asymptotic expansion of the weighted Bergman kernel for $(0, q)$ forms on $\mathbb{C}^{n}$, available at arXiv:1208.3818.

11. Lu, W., The second coefficient of the asymptotic expansion of the Bergman kernel of the Hodge-Dolbeault operator J. Geom. Anal., 25 (2015), 25-63.

12. Lu, Z., On the lower order terms of the asymptotic expansion of Tian-Yau-Zelditch, Amer. J. Math., 122 (2000), no.2, 235-273.

13. Ma, X. and Marinescu, G., The first coefficients of the asymptotic expansion of the Bergman kernel of the spin ${ }^{c}$ Dirac operator, Internat. J. Math, 17 (2006), 737-759.

14. Ma, X. and Marinescu, G., Holomorphic Morse Inequalities and Bergman Kernels, Progress in Math., Birkhäuser, Basel, 2007.

15. Ma, X. and Marinescu, G., Generalized Bergman kernels on symplectic manifolds, Adv. Math., 217 (2008), no.4, 1756-1815.

16. Ma, X. and Marinescu, G., Berezin-Toeplitz quantization on Kähler manifolds, $J$. Reine Angew. Math., 662 (2012), 1-56.

17. Tian, G., On a set of polarized Kähler metrics on algebraic manifolds, J. Differential Geom., 32 (1990), 99-130.

18. Zelditch, S., Szegö kernels and a theorem of Tian, Internat. Math. Res. Notices, 6 (1998), 317-331. 